\title{
Advanced prostate cancer risk, selenium, and oxidative stress: the role of genetic variation and environment
}

Citation for published version (APA):

Geybels, M. S. (2014). Advanced prostate cancer risk, selenium, and oxidative stress: the role of genetic variation and environment. [Doctoral Thesis, Maastricht University]. Datawyse / Universitaire Pers Maastricht. https://doi.org/10.26481/dis.20141029mg

Document status and date:

Published: 01/01/2014

DOI:

10.26481/dis.20141029mg

Document Version:

Publisher's PDF, also known as Version of record

\section{Please check the document version of this publication:}

- A submitted manuscript is the version of the article upon submission and before peer-review. There can be important differences between the submitted version and the official published version of record.

People interested in the research are advised to contact the author for the final version of the publication, or visit the DOI to the publisher's website.

- The final author version and the galley proof are versions of the publication after peer review.

- The final published version features the final layout of the paper including the volume, issue and page numbers.

Link to publication

\footnotetext{
General rights rights.

- You may freely distribute the URL identifying the publication in the public portal. please follow below link for the End User Agreement:

www.umlib.nl/taverne-license

Take down policy

If you believe that this document breaches copyright please contact us at:

repository@maastrichtuniversity.nl

providing details and we will investigate your claim.
}

Copyright and moral rights for the publications made accessible in the public portal are retained by the authors and/or other copyright owners and it is a condition of accessing publications that users recognise and abide by the legal requirements associated with these

- Users may download and print one copy of any publication from the public portal for the purpose of private study or research.

- You may not further distribute the material or use it for any profit-making activity or commercial gain

If the publication is distributed under the terms of Article $25 \mathrm{fa}$ of the Dutch Copyright Act, indicated by the "Taverne" license above, 


\section{ADVANCED PROSTATE CANCER RISK, SELENIUM, AND OXIDATIVE STRESS: The role of genetic variation and environment}

MILAN GEYBELS 
(C) Maastricht 2014, Milan S. Geybels

Advanced prostate cancer risk, selenium, and oxidative stress: the role of genetic variation and environment

ISBN 9789461593634

Layout: Milan Geybels

Cover: Milan Geybels and Datawyse - Universitaire Pers Maastricht

Printed by: Datawyse - Universitaire Pers Maastricht

Financial support for the printing of this thesis was kindly provided by the Dutch Cancer Society.

All rights reserved. No part of this thesis may be reproduced or transmitted in any form or by any means, electronic or mechanical, including photocopying, recording or any information storage or retrieval system, without permission in writing from the author, or, when appropriate, from the publishers of this publication. 


\title{
Advanced prostate cancer risk, selenium, and
}

\section{oxidative stress: the role of genetic variation and environment}

\author{
PROEFSCHRIFT \\ ter verkrijging van de graad van doctor aan de Universiteit Maastricht \\ op gezag van de Rector Magnificus, Prof. dr. L.L.G. Soete, \\ volgens het besluit van het College van Decanen, \\ in het openbaar te verdedigen \\ op woensdag 29 oktober 2014 om 12.00 uur \\ door \\ Milan Stefan Geybels
}

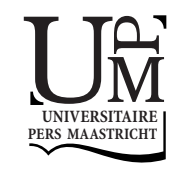




\section{Promotores}

Prof. dr. ir. P.A. van den Brandt

Prof. dr. F.J. van Schooten

\section{Copromotor}

Dr. B.A.J. Verhage

\section{Beoordelingscommissie}

Prof. dr. H.J.M. Smeets (voorzitter)

Prof. dr. A. Bast

Prof. dr. C.M. van Duijn (Universitair Medisch Centrum Rotterdam)

Dr. C.A. Hulsbergen-van de Kaa (Radboudumc Nijmegen)

Dr. J.G.H. van Roermund

This PhD research was supported by the Dutch Cancer Society (KWF).

The studies presented in this thesis were conducted at the GROW - School for Oncology and Developmental Biology at Maastricht University (department of Epidemiology) in collaboration with the NUTRIM - School for Nutrition, Toxicology, and Metabolism at Maastricht University (department of Toxicology). 


\section{Table of Contents}

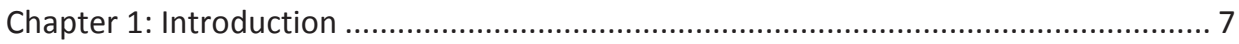

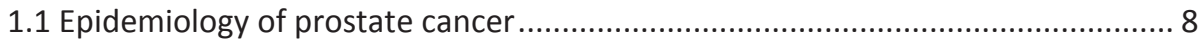

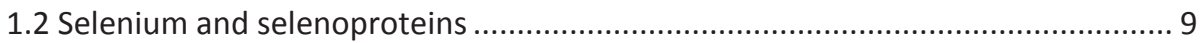

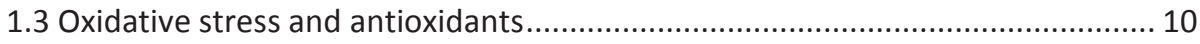

1.4 Selenium, other antioxidants, pro-oxidants, and prostate cancer risk ............... 12

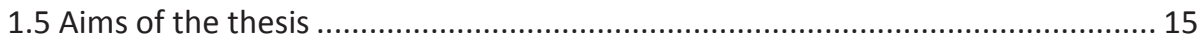

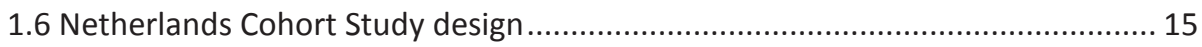

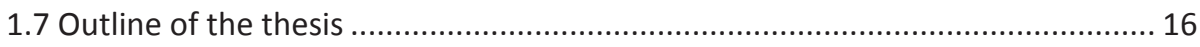

Chapter 2: Advanced Prostate Cancer Risk in Relation to Toenail Selenium Levels ...... 21

Chapter 3: Selenoprotein Gene Variants, Toenail Selenium Levels, and Risk of Advanced Prostate Cancer

Chapter 4: Measures of Combined Antioxidant and Pro-oxidant Exposures and Risk of Overall and Advanced Stage Prostate Cancer.

Chapter 5: Dietary Flavonoid Intake, Black Tea Consumption, and Risk of Overall and Advanced Stage Prostate Cancer.....

Chapter 6: Oxidative Stress-Related Genetic Variants, Pro- and Antioxidant Intake and Status, and Advanced Prostate Cancer Risk 95

Chapter 7: Discussion. 119

7.1 Main study findings 120

7.2 Interpretation of study findings 123

7.2.1 Selenium and prostate cancer risk - Is low selenium status a risk factor for prostate cancer?

7.2.2 Selenium and prostate cancer risk - A role for selenoprotein genes?....... 126

7.2.3 Oxidative stress and prostate cancer risk - A role for pro- and antioxidant intake?

7.2.4 Oxidative stress and prostate cancer - A role for genetic variation? ........ 130

7.3 Gene-environment interaction and exposure modification by genotype ........ 133

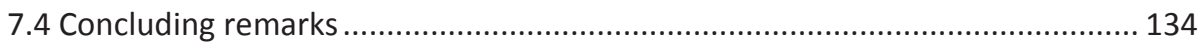

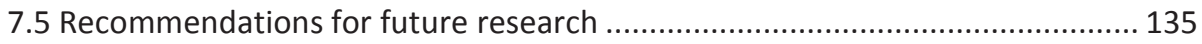

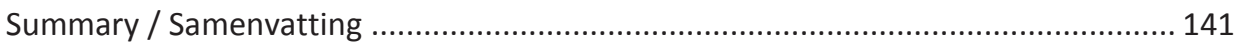

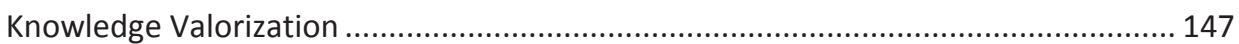

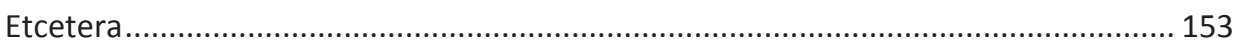



Chapter 1: Introduction 


\subsection{Epidemiology of prostate cancer}

Prostate cancer (PCa) is the most common cancer in men in Europe and the US [1, 2]. According to the most recent data approximately 1 in 7 US men will develop PCa during his lifetime [3]. In the Netherlands in 2011, 11,428 men were diagnosed with PCa and 2,500 men died as a result of the disease [4]. The incidence of PCa generally increases with advancing age $[5,6]$. It is relatively rare for PCa to be diagnosed in men before age 50, but after this age, incidence rates increase exponentially [7]. A worldwide increase in PCa incidence is expected as the male population ages [5].

The incidence of PCa is strongly influenced by early detection with the use of the serum prostate-specific antigen (PSA) test, one of the most commonly used clinical cancer tests [8, 9]. In 1994, the US Food and Drug Administration (FDA) approved the use of the PSA test in conjunction with a digital rectal exam to test asymptomatic men for PCa $[8,10]$. PSA testing has now become common practice in many developed countries. Based on results of the Statistics Netherlands survey, in 2012, 24.4\% of men older than age 39 in the Netherlands had their serum PSA measured in the previous 5 years [11]. This percentage was highest for men aged 65 to 75 years (44.4\%) and 75 or older (40.9\%).

PCa is a clinically heterogeneous disease with marked variability in patient outcomes [5, 12]. While many PCa patients will have slow-growing, indolent tumors that may never become clinically-relevant other patients will have aggressive disease associated with metastasis and death from PCa [13]. The frequent occurrence of indolent or latent PCa is confirmed by several autopsy studies that showed that many men who die from other causes than PCa have evidence of histological PCa (approximately $30-50 \%$ of men aged $50-70$ years) $[7,14,15]$. Since both PSA testing and latent PCa are common there is substantial overdiagnosis of PCa [16, 17]. It has been estimated that PCa overdiagnosis may occur in up to $67 \%$ of all cases [18]. Overdiagnosis of PCa is an important public health problem because it may lead to overtreatment. A consequence of PCa overtreatment is that many patients needlessly suffer from serious treatment-induced side effects (e.g., incontinence, impotence) [16].

Advanced PCa is a type of PCa associated with a poor prognosis that is therefore clinically relevant [19]. The TNM system is a widely accepted cancer staging system that is used to identify advanced PCa [20]. The TNM system is based on the size or extent of the primary tumor $(T)$, the amount of spread to nearby lymph nodes $(N)$, and the presence of metastasis (M) [3]. Advanced PCa typically involves those tumors that either extend beyond the prostate (T3-4), show positive lymph node involvement (N1), or are metastatic (M1) $[19,20]$. These cancers are classified by the International Union Against Cancer (UICC) as stage III and IV PCa [20]. Data from the Netherlands Cancer Registry (2003-2009) showed that while stage I/II prostate cancers have a 5- 
year survival rate of nearly $100 \%$, this is much lower for advanced cancers, in particular stage IV prostate cancers (45\%) [21]. Another prognostic marker of PCa is the Gleason Grading System, which is a predictor of PCa aggressiveness [22]. Gleason grade is based on cellular content and tissue architecture from biopsies and radical prostatectomy specimens and is typically used to identify aggressive rather than advanced PCa $[22,23]$.

Besides older age, other major risk factors for PCa are race and family history of PCa $[5,6,24]$. Incidence rates for men of African ancestry are nearly twice the incidence rates of their European and Asian counterparts [3]. PCa has shown to be one of the most hereditary cancers; the risk of developing the disease doubles for men with a first-degree relative affected by PCa and increases further with more affected relatives [25]. Evidence from twin studies indicates that heritable factors may explain as much as $42 \%$ of PCa risk $[26,27]$. No other established PCa risk factors have been identified and the causes of the disease remain poorly understood $[5,24,28]$. There is however increasing scientific evidence suggesting that the antioxidant nutrient selenium and the related oxidative stress pathway have a role in PCa [24, 29-31].

\subsection{Selenium and selenoproteins}

The trace mineral selenium is an essential micronutrient of fundamental importance to human biology $[32,33]$. The main dietary sources of selenium include fish, shellfish, cereals, meat, and dairy products [34]. In contrast to many other micronutrients, the intake of selenium varies hugely worldwide $[32,33]$. This variability in intake results from differences in selenium content of the soil on which crops are grown, and also differences in availability and chemical species of selenium in the soil [32]. Recommendations for selenium intake average $60 \mu \mathrm{g}$ per day for men and $53 \mu \mathrm{g}$ per day for women [33]. Selenium intakes are high in Venezuela, Canada, the US, and Japan. Europe is generally considered to have relatively low intakes [32]. China has areas of both selenium deficiency and excess [33]. Although the average selenium intake in the US is high, low selenium status may be common in certain US regions [35].

Selenium exerts biological functions through its presence in selenoproteins [36]. The human genome contains 25 selenoprotein genes. Selenoproteins are seleniumdependent and the element is incorporated in these proteins as the (the 21st) amino acid selenocysteine [37]. Selenocysteine is encoded by the UGA codon, which is normally a stop codon [36]. Selenoprotein synthesis is an evolutionary conserved process that depends on multiple protein and RNA factors such as the selenocysteine insertion sequence (SECIS), SECIS-binding protein 2 (SBP2), and selenocysteine-tRNA $[36,38]$. Although many selenoproteins have yet unknown functions, a number of 
biological activities of selenoproteins have been described. These functions include the reduction of thioredoxins (thioredoxin reductase), selenophosphate synthesis (selenophosphate synthetase), and activation and inactivation of thyroid hormones (iodothyronine deodinase) [36, 38]. Selenium, however, is best-known for its antioxidant activities that are achieved through incorporation in the selenoenzyme family of glutathione peroxidases (GPXs) [36, 39, 40]. GPX decomposes reactive oxygen species (ROS) that can cause oxidative stress and associated damage when levels become too high [39]. Another well-characterized selenoprotein is selenoprotein $P$ (SEPP1), a secreted glycoprotein that contains most of the selenium in plasma and is the main transport protein for selenium [41].

\subsection{Oxidative stress and antioxidants}

Reactive oxygen species, oxidative stress, and disease

Reactive oxygen species (ROS) are oxygen radicals and non-radicals that are produced as byproducts of normal cellular metabolism [42-44]. A free radical is any species capable of independent existence that contains one or more unpaired electrons [43]. Examples of oxygen free radicals are the superoxide radical $\left(\mathrm{O}_{2}{ }^{--}\right)$and the hydroxyl radical $\left(\mathrm{OH}^{\circ}\right)$. Major non-radical derivatives of oxygen include hydrogen peroxide $\left(\mathrm{H}_{2} \mathrm{O}_{2}\right)$ and singlet oxygen $\left({ }^{1} \mathrm{O}_{2}\right)$. An important endogenous source of ROS is the mitochondrial electron transport chain [45]. Other endogenous factors that generate reactive species include inflammatory processes, nitric oxide synthases (NOS), and lipid peroxidation [44]. ROS are also produced by exposure to exogenous pro-oxidants such as radiation, metal ions, cigarette smoke, ethanol, and some drugs [44]. While moderate concentrations of ROS are important for normal cellular functioning (e.g., cellular signaling), elevated concentrations of ROS are damaging and trigger a condition called oxidative stress [42-44]. This condition is associated with excessive oxidative damage to different cellular constituents (e.g., proteins, lipids, nucleic acids). Oxidative stress has been implicated in aging $[46,47]$, and is believed to play a role in age-related degenerative diseases including PCa [24, 30, 42, 48, 49]. Figure 1.1 presents a scheme of the endo- and exogenous sources of ROS, associated cellular responses, and the potential link with $\mathrm{PCa}$. 


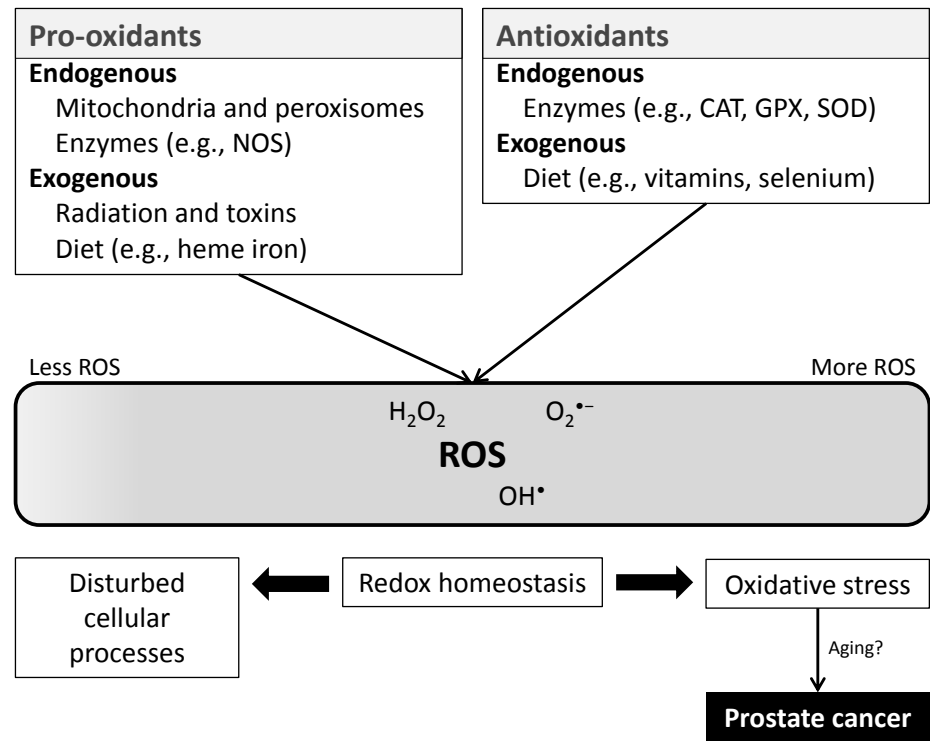

Figure 1.1

Endo- and exogenous sources of reactive oxygen species, associated cellular responses, and the potential link with prostate cancer

Abbreviations: reactive oxygen species, ROS; catalase, CAT; glutathione peroxidase, GPX; superoxide dismutase, SOD; nitric oxidase synthase, NOS

\section{Antioxidant enzymes and nutrients}

The burden of increased ROS production is counteracted by an intricate antioxidant defense system [42-44,50]. Antioxidants are molecules that detoxify oxygen radicals and other ROS and thereby protect against oxidative stress. The antioxidant system includes both enzymes and dietary nutrients (Figure 1.1) [44, 50].

Key antioxidant enzymes are glutathione peroxidase (GPX), catalase (CAT), and superoxide dismutase (SOD) [44, 50]. GPXs are selenoproteins that have selenium as selenocysteine at their active site [39]. These enzymes catalyze the reduction of $\mathrm{H}_{2} \mathrm{O}_{2}$ and other hydroperoxides using glutathione (Figure 1.2) [39]. There are 5 known selenium-dependent GPXs in humans, i.e. GPX1-4 and GPX6 [39]. Although their expression is ubiquitous, the levels of each isoform vary depending on the tissue type [50]. Catalase (CAT) is an enzyme present in the cell's peroxisome where large amounts of ROS are produced [44, 50]. Like GPX, CAT also reacts with $\mathrm{H}_{2} \mathrm{O}_{2}$, which it reduces to water and oxygen (Figure 1.2). CAT has one of the highest turnover rates of all enzymes; one molecule of CAT can convert about 6 million molecules of $\mathrm{H}_{2} \mathrm{O}_{2}$ per minute [44]. SOD catalyzes the dismutation of $\mathrm{O}_{2}{ }^{--}$to oxygen and to the less-reactive $\mathrm{H}_{2} \mathrm{O}_{2}$ (Figure 1.2) $[44,50]$. SOD exists in several isoforms that differ in the nature of the active metal center. In humans there are three forms of SOD, i.e.: cytosolic $\mathrm{Cu}, \mathrm{Zn}$-SOD 
(SOD1); mitochondrial Mn-SOD (SOD2); and extracellular SOD (SOD3). Because SODs generate $\mathrm{H}_{2} \mathrm{O}_{2}$, they have to work together with GPXs and CAT to remove $\mathrm{H}_{2} \mathrm{O}_{2}$ [43]. Besides these major antioxidant enzymes, there are a number of other enzymes with potentially important antioxidant activities (e.g., NADPH-quinone oxidoreductase, paraoxonase) $[50,51]$.

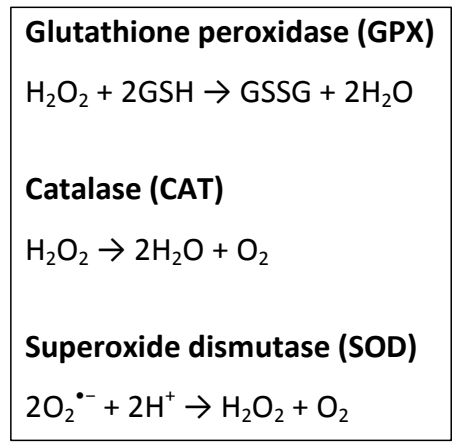

Figure 1.2

Chemical reactions of the main antioxidant enzymes glutathione peroxidase, catalase, and superoxide dismutase

Major antioxidant nutrients include vitamins $C$ and $E$, carotenoids (e.g., $\beta$ carotene and lycopene), flavonoids (e.g., catechins and flavonols), and selenium [44, 52]. Vitamin C (ascorbic acid) works as an antioxidant in aqueous environments. It acts both directly by reaction with ROS and indirectly by restoring the antioxidant properties of vitamin E [44]. Vitamin E is a fat-soluble vitamin that inhibits ROSinduced generation of lipid peroxyl radicals, thereby protecting against lipid peroxidation [52]. Vitamin E exists in eight different forms and $\alpha$-tocopherol is the most active form in humans [44]. Approximately 600 different carotenoids have been described. The antioxidant properties of carotenoids are associated with their radical scavenging properties and their ability to quench ${ }^{1} \mathrm{O}_{2}$ [53]. Flavonoids are another large family of naturally occurring compounds, with more than 5,000 members having been described. The antioxidant capacity of flavonoids is due to their ability to reduce free radical formation and to scavenge free radicals $[44,54]$. The antioxidant activities of selenium are mainly attributed to the antioxidant enzymes GPX, which are seleniumdependent [44].

\subsection{Selenium, other antioxidants, pro-oxidants, and prostate cancer risk}

There have been a number of epidemiological studies that investigated the link between pro- and antioxidants and PCa risk. These investigations focused on both exogenous (external) and endogenous (internal) factors. Exogenous factors include 
various antioxidant nutrients including selenium, carotenoids, and vitamins; but also pro-oxidants exposures like smoking and dietary factors [42, 44, 55]. The studies on endogenous pro- and antioxidants focused on genetic variants. Variation in genes encoding pro- and antioxidant enzymes may influence oxidative stress levels and cellular responses [56].

\section{Selenium}

Selenium has long been thought to have anticarcinogenic properties [31, 57, 58], and a chemopreventive effect of selenium has been demonstrated in a large randomized study: the Nutritional Prevention of Cancer (NPC) trial [59]. This study recruited 1,312 volunteers with a previous history of non-melanoma skin cancer from the southeastern US, a region with low selenium soil concentrations. Participants were randomized from 1983 to 1991. The study showed that treatment with $200 \mu \mathrm{g}$ selenium per day (as selenium yeast) had no effect on the primary outcome of nonmelanoma skin cancer, but in a secondary analysis the study found that selenium supplementation reduced the risk of total cancer (25\%) and PCa $(52 \%)[29,60,61]$. Furthermore, the association between selenium levels and PCa risk has been studied in a number of prospective analyses. These observational studies generally support an association between higher selenium levels and lower PCa risk [29].

The effect of selenium supplementation on PCa incidence was further investigated in the Selenium and Vitamin E Cancer Prevention Trial (SELECT) [62]. SELECT is a phase 3 randomized, placebo-controlled trial that included 35,533 American men. Participants were randomized from 2001 to 2004. The study showed that selenium supplementation (as selenomethionine; $200 \mu \mathrm{g} /$ day) did not reduce PCa risk $[62,63]$. The null result for selenium in SELECT was surprising given the substantial scientific evidence from earlier studies suggesting that selenium reduces PCa risk [29, 60]. A number of possible explanations for these disparate findings have been proposed, in particular that baseline selenium levels in SELECT may have been too high for additional selenium intake to have a beneficial effect [33, 64-66]. Compared to the NPC trial, which was conducted among subjects from regions in the US with low selenium soil concentrations [59], SELECT included American men with relatively high baseline selenium (median plasma selenium level = $136 \mu \mathrm{g} / \mathrm{L}$ ) [62]. This hypothesis is further strengthened by the observation that in the NPC trial selenium supplementation only reduced PCa risk among subjects in the two lowest tertiles of baseline selenium ( $<123 \mu \mathrm{g} / \mathrm{L}$ plasma selenium) [60]. Other possible explanations for the mixed results on selenium and PCa risk include differences in selenium species (in foods and supplements) and genetic variation in selenoprotein genes and related pathways [33, 64-66]. 


\section{Other antioxidant nutrients and pro-oxidant exposures}

Besides selenium other antioxidant nutrients have been investigated in relation to $\mathrm{PCa}$ risk. The Alpha-Tocopherol, Beta-Carotene Cancer Prevention (ATBC) Study was designed to study the effect of vitamin E (as $\alpha$-tocopherol) and $\beta$-carotene supplementation on the risk of lung cancer (primary endpoint) and other cancers (secondary endpoint) [67]. The trial included 29,133 male smokers from Finland and randomization took place from 1985 to 1988. The study showed that supplementation with vitamin $E$ and $\beta$-carotene did not reduce lung cancer risk, but found that vitamin $E$ supplementation lowered PCa risk $[67,68]$. The preventive effect on PCa risk was, however, not significant after additional follow-up of the study population [69]. SELECT not only studied selenium but also vitamin E and, surprisingly, the trial showed that supplementation with vitamin E (as $\alpha$-tocopherol) significantly increased PCa risk [63]. A number of prospective and case-control studies investigated antioxidant nutrients (intake or levels) in relation to PCa risk. These studies generally do not support an association with the nutrients investigated including vitamins $C$ and $E, \beta$-carotene, and lycopene $[55,70]$. A number of observational studies examined the associations between PCa risk and pro-oxidant exposures such as smoking [71], alcohol [72], and dietary iron $[73,74]$, but these studies generally do not support an association.

\section{Genetic variation in oxidative stress-related genes}

Genetic variation in genes encoding antioxidant enzymes and other oxidative stressrelated proteins may influence oxidative stress levels and responses. A number of previous candidate gene association studies investigated the associations between oxidative stress-related gene variants and PCa risk. These studies focused on single nucleotide polymorphism (SNPs), which represent differences in single nucleotides and are the most common type of genetic variation in humans [75]. When SNPs occur within a gene or in a regulatory region near a gene, they may play a role in disease by affecting the gene's function. The previous studies on oxidative stress-related genetic variation and $\mathrm{PCa}$ risk showed significant associations with variants in several candidate genes such as SOD2, NOS2A, PON1, and hOGG1 [76-79]. These findings require confirmation from other studies because most associations were only reported in one or two small studies. Furthermore, most previous studies only investigated total PCa but not advanced PCa.

The antioxidant system is a dynamic and redundant system that involves many endo- and exogenous components that protect against ROS produced by various inand external sources [42-44]. It is, therefore, reasonable to assume that pro- and antioxidant genes and exposures do not merely act independently, but also have combined effects via gene-environment interactions. Studies of gene-environment interactions aim to describe how genetic and environmental factors jointly influence 
disease risk [80, 81]. Relatively few epidemiological studies searched for interactions between oxidative stress-related gene variants and exposures on PCa risk. These studies showed some significant findings including interactions between SOD2 and selenium [82], and hOGG1 and $\alpha$-tocopherol [79]. These findings require confirmation from other studies.

\subsection{Aims of the thesis}

The overall aim of the research described in this thesis was to investigate the link between selenium, selenoproteins, oxidative stress, and advanced PCa risk. In the first part of the thesis we examined how selenium status and selenoprotein genes impact PCa risk. The hypotheses were that low toenail selenium levels are inversely associated with advanced PCa risk, and selenoprotein gene variants modify advanced PCa risk and the selenium-PCa association. In the second part of the thesis we examined how various pro- and antioxidant genes and exposures impact PCa risk. We hypothesized that pro- and antioxidant exposures, oxidative stress-related gene variants, and gene-environment interactions between these two factors modify (advanced) PCa risk.

\subsection{Netherlands Cohort Study design}

The different analyses in this thesis were conducted within the prospective Netherlands Cohort Study [83]. The Netherlands Cohort Study included 58,279 men and 62,573 women, aged 55 to 69 years at baseline in September 1986. Netherlands Cohort Study participants completed a detailed baseline questionnaire on many potential cancer risk factors and provided toenail clippings, which were used to determine selenium concentrations and to isolate DNA for genotyping. Cancer cases were prospectively identified by annual record linkage to the Netherlands Cancer Registry and the Dutch Pathology Registry [84]. Clinical and pathological data from these registries were used to identify advanced PCa cases from all PCa cases. For the research in this thesis, a follow-up of 17.3 years was used. The thesis specifically focuses on advanced PCa, which is clinically relevant because it has a poor prognosis [19]. We used the UICC TNM system to classify PCa [20]. Two definitions of advanced PCa were used in our analyses: (1) UICC stage III/IV PCa, which is T3-4, N+, or M1 (i.e., T3NOMO; T4NOMO; T[any]N+MO; T[any]N[any]M1); and (2) UICC stage IV PCa, which is $\mathrm{T} 4, \mathrm{~N}+$, or $\mathrm{M} 1$ (i.e., T4NOM0; T[any]N+M0; T[any]N[any]M1). In our analyses, both definitions of advanced PCa were used. TNM information was available for the majority of cases in our study (94\%). 
The case-cohort design

The Netherlands Cohort Study employs a case-cohort design [85]. This type of nested design involves selecting a single subcohort from the initial cohort at inception ( $\mathrm{n}=$ 2,411 male Netherlands Cohort Study participants), and adding all the cases that occur in the cohort (outside the subcohort). The subcohort is used to estimate the persontime experience [85]. The advantage of this design is that it considerably reduces data collection, which is important because for our research we used costly and timeconsuming methods for exposure monitoring and genotyping [86]. The case-cohort design is similar to the nested case-control design, which however can be inefficient when a cohort study is used to investigate multiple outcomes as is the case in the Netherlands Cohort Study.

Selection of single nucleotide polymorphisms, DNA isolation, and genotyping

For the research described in this thesis we utilized a candidate gene association approach. This approach involves analyzing single nucleotide polymorphisms (SNPs), which represent the most common type of genetic variation in humans [75]. We studied SNPs in candidate genes encoding major selenoproteins (SEPP1, GPX1) and other oxidative stress-related proteins (e.g., CAT, SOD2, hOGG1, NOS2A). Two different SNP selection strategies were used in this thesis; i.e., selecting (1) tagging SNPs to capture all common variation in genes (selenoprotein genes) and, (2) candidate SNPs that have reported functionality or have been associated with PCa risk in previous studies (oxidative stress-related genes). SNP genotyping was done using DNA isolated from toenail clippings of study participants. Our group previously showed that stored toenail samples of Netherlands Cohort Study participants are a stable and very useful source of DNA for SNP genotyping [87]. Genotyping was done using Sequenom's MassARRAY ${ }^{\circledR}$ technology (Sequenom, Hamburg, Germany).

\subsection{Outline of the thesis}

In Chapter 2 of this thesis we studied the association between toenail selenium levels and advanced PCa risk. In Chapter 3 we investigated whether SEPP1 and GPX1 genetic variants were associated with advanced PCa risk and modified the association between toenail selenium levels and advanced PCa risk. In Chapter 4 and 5 we examined the associations between major pro- and antioxidant exposures and advanced PCa risk. In Chapter 6 we investigated the associations between oxidative stress-related genetic variants and advanced PCa risk and the gene-environment interactions with pro- and antioxidant exposures. Finally, Chapter 7 provides a general discussion of the thesis. 


\section{References}

1. Ferlay J, Steliarova-Foucher E, Lortet-Tieulent J, et al. Cancer incidence and mortality patterns in Europe: estimates for 40 countries in 2012. European journal of cancer 2013;49(6):1374-1403.

2. Siegel R, Naishadham D, Jemal A. Cancer statistics, 2013. CA: a cancer journal for clinicians 2013;63(1):11-30.

3. cancer.org [homepage on the internet]. American Cancer Society [cited 2 June 2014]. Available from: http://www.cancer.org/cancer/prostatecancer/detailedguide/prostate-cancer-key-statistics.

4. cijfersoverkanker.nl [homepage on the internet]. Nederlandse Kankerregistratie [cited 2 June 2014]. Available from: http://www.cijfersoverkanker.nl/selecties/Incidentie_prostaatkanker/ img534e82187ec19.

5. Crawford ED. Understanding the epidemiology, natural history, and key pathways involved in prostate cancer. Urology 2009;73(5 Suppl):S4-10.

6. Gronberg H. Prostate cancer epidemiology. Lancet 2003;361(9360):859-864.

7. Haas GP, Sakr WA. Epidemiology of prostate cancer. CA: a cancer journal for clinicians 1997;47(5):273287.

8. Hernandez J, Thompson IM. Prostate-specific antigen: a review of the validation of the most commonly used cancer biomarker. Cancer 2004;101(5):894-904.

9. Balk SP, Ko YJ, Bubley GJ. Biology of prostate-specific antigen. Journal of clinical oncology : official journal of the American Society of Clinical Oncology 2003;21(2):383-391.

10. Roobol MJ. The prostate-specific antigen test. Expert Opin Med Diagn 2013;7(5):423-426.

11. statline.cbs.nl [homepage on the internet]. Centraal Bureau voor de Statistiek [cited 2 June 2014]. Available from: http://statline.cbs.nl/StatWeb/publication/?DM=SLNL\&PA=81177ned\&D1=45-47\&D2=013,26-27,30,33-37\&D3=0\&D4=I\&VW=T.

12. Barbieri $\mathrm{CE}$, Bangma $\mathrm{CH}, \mathrm{Bjartell} \mathrm{A}$, et al. The mutational landscape of prostate cancer. European urology 2013;64(4):567-576.

13. Punnen S, Cooperberg MR. The epidemiology of high-risk prostate cancer. Current opinion in urology 2013;23(4):331-336.

14. Sakr WA, Haas GP, Cassin BF, et al. The frequency of carcinoma and intraepithelial neoplasia of the prostate in young male patients. The Journal of urology 1993;150(2 Pt 1):379-385.

15. Zlotta AR, Egawa S, Pushkar D, et al. Prevalence of prostate cancer on autopsy: cross-sectional study on unscreened Caucasian and Asian men. Journal of the National Cancer Institute 2013;105(14):1050-1058.

16. Klotz L. Prostate cancer overdiagnosis and overtreatment. Current opinion in endocrinology, diabetes, and obesity 2013;20(3):204-209.

17. Welch HG, Black WC. Overdiagnosis in cancer. Journal of the National Cancer Institute 2010;102(9):605613.

18. Loeb S, Bjurlin MA, Nicholson J, et al. Overdiagnosis and Overtreatment of Prostate Cancer. European urology 2014;65(6):1046-1055.

19. Bostwick DG, Grignon DJ, Hammond ME, et al. Prognostic factors in prostate cancer. College of American Pathologists Consensus Statement 1999. Arch Pathol Lab Med 2000;124(7):995-1000.

20. Sobin L, Gospodarowicz M, Wittekind C, et al. TNM Classification of Malignant Tumors. 7th ed. Oxford, UK: Wiley-Blackwell; 2009.

21. cijfersoverkanker.nl [homepage on the internet]. Nederlandse Kankerregistratie [cited 2 June 2014]. Available from: https://www.kanker.nl/bibliotheek/prostaatkanker/wat-is/1095-cijfers-overprostaatkanker.

22. Lavery HJ, Droller MJ. Do Gleason patterns 3 and 4 prostate cancer represent separate disease states? The Journal of urology 2012;188(5):1667-1675.

23. DeMarzo AM, Nelson WG, Isaacs WB, et al. Pathological and molecular aspects of prostate cancer. Lancet 2003;361(9361):955-964.

24. Bostwick DG, Burke HB, Djakiew D, et al. Human prostate cancer risk factors. Cancer 2004;101(10 Suppl):2371-2490.

25. Kicinski M, Vangronsveld J, Nawrot TS. An epidemiological reappraisal of the familial aggregation of prostate cancer: a meta-analysis. PloS one 2011;6(10):e27130.

26. Lichtenstein P, Holm NV, Verkasalo PK, et al. Environmental and heritable factors in the causation of cancer--analyses of cohorts of twins from Sweden, Denmark, and Finland. The New England journal of medicine 2000;343(2):78-85. 
27. Page WF, Braun MM, Partin AW, et al. Heredity and prostate cancer: a study of World War II veteran twins. The Prostate 1997;33(4):240-245.

28. Nelson WG, De Marzo AM, Isaacs WB. Prostate cancer. The New England journal of medicine 2003;349(4):366-381.

29. Hurst R, Hooper L, Norat T, et al. Selenium and prostate cancer: systematic review and meta-analysis. The American journal of clinical nutrition 2012;96(1):111-122.

30. Minelli A, Bellezza I, Conte C, et al. Oxidative stress-related aging: A role for prostate cancer? Biochimica et biophysica acta 2009;1795(2):83-91.

31. Peters U, Takata Y. Selenium and the prevention of prostate and colorectal cancer. Molecular nutrition $\&$ food research 2008;52(11):1261-1272.

32. Fairweather-Tait SJ, Bao YP, Broadley MR, et al. Selenium in Human Health and Disease. Antioxidants \& redox signaling 2011;14(7):1337-1383.

33. Rayman MP. Selenium and human health. Lancet 2012;379(9822):1256-1268.

34. Hurst R, Collings R, Harvey LJ, et al. EURRECA-Estimating Selenium Requirements for Deriving Dietary Reference Values. Critical Reviews in Food Science and Nutrition 2013;53(10):1077-1096.

35. Park K, Rimm E, Siscovick D, et al. Demographic and lifestyle factors and selenium levels in men and women in the U.S. Nutrition research and practice 2011;5(4):357-364.

36. Papp LV, Lu J, Holmgren A, et al. From selenium to selenoproteins: synthesis, identity, and their role in human health. Antioxidants \& redox signaling 2007;9(7):775-806.

37. Hatfield DL, Gladyshev VN. How selenium has altered our understanding of the genetic code. Mol Cell Biol 2002;22(11):3565-3576.

38. Hatfield DL, Tsuji PA, Carlson BA, et al. Selenium and selenocysteine: roles in cancer, health, and development. Trends Biochem Sci 2014.

39. Brigelius-Flohe R, Kipp A. Glutathione peroxidases in different stages of carcinogenesis. Biochimica et biophysica acta 2009;1790(11):1555-1568.

40. Rayman MP. The importance of selenium to human health. Lancet 2000;356(9225):233-241.

41. Burk RF, Hill KE. Selenoprotein P-Expression, functions, and roles in mammals. Biochimica Et Biophysica Acta-General Subjects 2009;1790(11):1441-1447.

42. Droge W. Free radicals in the physiological control of cell function. Physiol Rev 2002;82(1):47-95.

43. Halliwell B. Reactive species and antioxidants. Redox biology is a fundamental theme of aerobic life. Plant Physiol 2006;141(2):312-322.

44. Valko M, Rhodes CJ, Moncol J, et al. Free radicals, metals and antioxidants in oxidative stress-induced cancer. Chemico-biological interactions 2006;160(1):1-40.

45. Cadenas E, Davies KJ. Mitochondrial free radical generation, oxidative stress, and aging. Free radical biology \& medicine 2000;29(3-4):222-230.

46. Finkel T, Holbrook NJ. Oxidants, oxidative stress and the biology of ageing. Nature 2000;408(6809):239247.

47. Muller FL, Lustgarten MS, Jang $\mathrm{Y}$, et al. Trends in oxidative aging theories. Free Radical Biology and Medicine 2007;43(4):477-503.

48. Halliwell B. Oxidative stress and cancer: have we moved forward? The Biochemical journal 2007;401(1):1-11.

49. Khandrika L, Kumar B, Koul S, et al. Oxidative stress in prostate cancer. Cancer letters 2009;282(2):125136.

50. Mates JM. Effects of antioxidant enzymes in the molecular control of reactive oxygen species toxicology. Toxicology 2000;153(1-3):83-104.

51. Mackness MI, Mackness B, Durrington PN, et al. Paraoxonase: biochemistry, genetics and relationship to plasma lipoproteins. Current opinion in lipidology 1996;7(2):69-76.

52. Fang YZ, Yang S, Wu G. Free radicals, antioxidants, and nutrition. Nutrition 2002;18(10):872-879.

53. El-Agamey A, Lowe GM, McGarvey DJ, et al. Carotenoid radical chemistry and antioxidant/pro-oxidant properties. Arch Biochem Biophys 2004;430(1):37-48.

54. Pietta PG. Flavonoids as antioxidants. J Nat Prod 2000;63(7):1035-1042.

55. Vance TM, Su J, Fontham ET, et al. Dietary antioxidants and prostate cancer: a review. Nutrition and cancer 2013;65(6):793-801.

56. Crawford A, Fassett RG, Geraghty DP, et al. Relationships between single nucleotide polymorphisms of antioxidant enzymes and disease. Gene 2012;501(2):89-103. 
57. Brigelius-Flohe R. Selenium compounds and selenoproteins in cancer. Chem Biodivers 2008;5(3):389395.

58. Rayman MP. Selenium in cancer prevention: a review of the evidence and mechanism of action. The Proceedings of the Nutrition Society 2005;64(4):527-542.

59. Clark LC, Combs GF, Turnbull BW, et al. Effects of selenium supplementation for cancer prevention in patients with carcinoma of the skin a randomized controlled trial - A randomized controlled trial. JamaJournal of the American Medical Association 1996;276(24):1957-1963.

60. Duffield-Lillico AJ, Dalkin BL, Reid ME, et al. Selenium supplementation, baseline plasma selenium status and incidence of prostate cancer: an analysis of the complete treatment period of the Nutritional Prevention of Cancer Trial. BJU international 2003;91(7):608-612.

61. Duffield-Lillico AJ, Reid ME, Turnbull BW, et al. Baseline characteristics and the effect of selenium supplementation on cancer incidence in a randomized clinical trial: a summary report of the Nutritional Prevention of Cancer Trial. Cancer epidemiology, biomarkers \& prevention : a publication of the American Association for Cancer Research, cosponsored by the American Society of Preventive Oncology 2002;11(7):630-639.

62. Lippman SM, Klein EA, Goodman PJ, et al. Effect of selenium and vitamin E on risk of prostate cancer and other cancers: the Selenium and Vitamin E Cancer Prevention Trial (SELECT). JAMA : the journal of the American Medical Association 2009;301(1):39-51.

63. Klein EA, Thompson IM, Jr., Tangen CM, et al. Vitamin E and the risk of prostate cancer: the Selenium and Vitamin E Cancer Prevention Trial (SELECT). JAMA : the journal of the American Medical Association 2011;306(14):1549-1556.

64. Meplan C, Hesketh J. Selenium and Cancer: A Story that Should not be Forgotten-Insights from Genomics. Cancer Treat Res 2014;159:145-166.

65. Nicastro HL, Dunn BK. Selenium and Prostate Cancer Prevention: Insights from the Selenium and Vitamin E Cancer Prevention Trial (SELECT). Nutrients 2013;5(4):1122-1148.

66. Steinbrenner H, Speckmann B, Sies H. Toward Understanding Success and Failures in the Use of Selenium for Cancer Prevention. Antioxidants \& redox signaling 2013;19(2):181-191.

67. The effect of vitamin $E$ and beta carotene on the incidence of lung cancer and other cancers in male smokers. The Alpha-Tocopherol, Beta Carotene Cancer Prevention Study Group. The New England journal of medicine 1994;330(15):1029-1035.

68. Heinonen OP, Albanes D, Virtamo J, et al. Prostate cancer and supplementation with alpha-tocopherol and beta-carotene: incidence and mortality in a controlled trial. Journal of the National Cancer Institute 1998;90(6):440-446.

69. Virtamo J, Pietinen P, Huttunen JK, et al. Incidence of cancer and mortality following alpha-tocopherol and beta-carotene supplementation: a postintervention follow-up. JAMA : the journal of the American Medical Association 2003;290(4):476-485.

70. Wei MY, Giovannucci EL. Lycopene, Tomato Products, and Prostate Cancer Incidence: A Review and Reassessment in the PSA Screening Era. J Oncol 2012;2012:271063.

71. Zu K, Giovannucci E. Smoking and aggressive prostate cancer: a review of the epidemiologic evidence. Cancer causes \& control : CCC 2009;20(10):1799-1810.

72. Rota M, Scotti L, Turati F, et al. Alcohol consumption and prostate cancer risk: a meta-analysis of the dose-risk relation. Eur J Cancer Prev 2012;21(4):350-359.

73. Choi JY, Neuhouser ML, Barnett MJ, et al. Iron intake, oxidative stress-related genes (MnSOD and MPO) and prostate cancer risk in CARET cohort. Carcinogenesis 2008;29(5):964-970.

74. Jakszyn PG, Allen NE, Lujan-Barroso L, et al. Nitrosamines and heme iron and risk of prostate cancer in the European prospective investigation into cancer and nutrition. Cancer epidemiology, biomarkers \& prevention : a publication of the American Association for Cancer Research, cosponsored by the American Society of Preventive Oncology 2012;21(3):547-551.

75. www.nih.gov.

76. Kang D, Lee KM, Park SK, et al. Functional variant of manganese superoxide dismutase (SOD2 V16A) polymorphism is associated with prostate cancer risk in the prostate, lung, colorectal, and ovarian cancer study. Cancer epidemiology, biomarkers \& prevention : a publication of the American Association for Cancer Research, cosponsored by the American Society of Preventive Oncology 2007;16(8):15811586 .

77. Stevens VL, Rodriguez C, Talbot JT, et al. Paraoxonase 1 (PON1) polymorphisms and prostate cancer in the CPS-II Nutrition Cohort. The Prostate 2008;68(12):1336-1340. 
78. Lee KM, Kang D, Park SK, et al. Nitric oxide synthase gene polymorphisms and prostate cancer risk. Carcinogenesis 2009;30(4):621-625.

79. Zhang J, Dhakal IB, Greene G, et al. Polymorphisms in hOGG1 and XRCC1 and risk of prostate cancer: effects modified by plasma antioxidants. Urology 2010;75(4):779-785.

80. Thomas D. Gene--environment-wide association studies: emerging approaches. Nature reviews. Genetics 2010;11(4):259-272.

81. Hunter DJ. Gene-environment interactions in human diseases. Nature reviews. Genetics 2005;6(4):287298.

82. Li H, Kantoff PW, Giovannucci E, et al. Manganese superoxide dismutase polymorphism, prediagnostic antioxidant status, and risk of clinical significant prostate cancer. Cancer research 2005;65(6):24982504.

83. van den Brandt PA, Goldbohm RA, van 't Veer $\mathrm{P}$, et al. A large-scale prospective cohort study on diet and cancer in The Netherlands. Journal of clinical epidemiology 1990;43(3):285-295.

84. van den Brandt PA, Schouten LJ, Goldbohm RA, et al. Development of a record linkage protocol for use in the Dutch Cancer Registry for epidemiological research. International journal of epidemiology 1990;19(3):553-558.

85. Barlow WE, Ichikawa L, Rosner D, et al. Analysis of case-cohort designs. Journal of clinical epidemiology 1999;52(12):1165-1172.

86. Cologne J, Preston DL, Imai K, et al. Conventional case-cohort design and analysis for studies of interaction. International journal of epidemiology 2012;41(4):1174-1186.

87. van Breda SG, Hogervorst JG, Schouten L, et al. Toenails: an easily accessible and long-term stable source of DNA for genetic analyses in large-scale epidemiological studies. Clinical chemistry 2007;53(6):1168-1170. 


\title{
Chapter 2: Advanced Prostate Cancer Risk in Relation to Toenail Selenium Levels
}

\author{
Milan S. Geybels \\ Bas A.J. Verhage \\ Frederik J. van Schooten \\ R. Alexandra Goldbohm
}

Piet A. van den Brandt

Journal of the National Cancer Institute 2013;105(18):1394-1401 


\begin{abstract}
Background

Selenium may prevent advanced prostate cancer (PCa), but most studies on this topic were conducted in populations with moderate to high selenium status. We investigated the association of toenail selenium, reflecting long-term selenium exposure, and advanced PCa risk in a population from the Netherlands where low selenium status is widespread.
\end{abstract}

\title{
Methods
}

The analysis was conducted in the prospective Netherlands Cohort study, which included 58,279 men aged 55 to 69 years at baseline in 1986. All cohort members completed a baseline questionnaire and $79 \%$ of participants provided toenail clippings, which were used for toenail selenium measurements using instrumental neutron activation analysis. Incident advanced PCa cases from the entire cohort were identified during 17.3 years of follow-up. The study employed a case-cohort design for which a random subcohort was sampled at baseline. Hazard ratios (HRs) and 95\% confidence intervals (Cls) were estimated using Cox proportional hazards regression models. All tests were two-sided.

\section{Results}

Complete toenail selenium data were available for 898 advanced (UICC stage III/IV) PCa cases and 1,176 subcohort members. The average toenail selenium concentration of subcohort members was $0.550 \mu \mathrm{g} / \mathrm{g}$. Toenail selenium was associated with a reduced risk of advanced $\mathrm{PCa}$; adjusted $\mathrm{HR}$ for the highest versus lowest quintile was 0.37 (95\% Cl: 0.27, 0.51; P for trend <0.001). For stage IV PCa, men in the highest versus lowest quintile of toenail selenium had an adjusted HR of 0.30 ( $95 \% \mathrm{Cl}: 0.21$, 0.45; $P$ for trend $<0.001$ ).

\section{Conclusion}

Toenail selenium was associated with a substantial decrease in risk of advanced PCa. 


\section{Introduction}

Selenium is an essential micronutrient that is incorporated into proteins as part of the amino acid selenocysteine and exerts important biological functions through its presence in these selenoproteins [1, 2]. The intake of selenium varies hugely worldwide as a result of global variations in the selenium soil content and related variability in the selenium content of foods $[3,4]$. While the average selenium intake is high in the US, low selenium intake is estimated to be widespread in Europe [3, 4]. Selenium-containing supplements also add to these intakes, especially in the US where dietary supplements are commonly used [4]. Within the US, however, selenium intake may vary substantially and low selenium status may be common in certain US regions [5].

The association of selenium and PCa has been studied in a number of prospective studies with some analyses showing an inverse association, particularly for advanced PCa, and others showing no relationship [6]. Interestingly, the association has been mainly studied over a relatively narrow range of selenium with levels from moderate to high. Most studies were conducted in populations from the US that did not include men with low selenium status who are at higher risk of less-overt selenium-deficiency $[3,6]$. Most studies of selenium and PCa risk used blood for exposure monitoring, but selenium concentrations can also be measured in toenails [6]. While blood selenium reflects recent exposures in the order of weeks, toenail selenium reflects exposures that have occurred over six months to one year and is, therefore, ideal for monitoring long-term exposure $[6,7]$. Three prospective studies of toenail selenium and PCa risk have been conducted and one of these studies is a previous analysis in the Netherlands Cohort Study, which had a follow-up for incident PCa of 6.3 years [8]. The analysis showed an association with a reduced risk of overall PCa and a statistically nonsignificant association with a reduced risk of advanced PCa. The study included only 183 advanced PCa cases and, therefore, had limited power to study the relationship. Also, due to the limited follow-up time in the study, the potential effect of long-term follow-up on the association could not be examined. The two other prospective studies of toenail selenium and PCa also had a small number of cases and a limited follow-up [6].

Despite the evidence from large prospective studies of an inverse association between selenium and PCa [6], such a relationship is not supported by the Selenium and Vitamin E Cancer Prevention Trial (SELECT) [9, 10]. The clinical trial among American men showed no association of selenium supplementation and PCa incidence after a median follow-up of seven years [10]. One possible explanation for the null result is the high median baseline selenium level of men in SELECT $(136 \mu \mathrm{g} / \mathrm{L})[4,11$, 12]. In an earlier clinical trial, it has been shown that selenium supplementation to 
men with blood selenium concentrations of more than $122 \mu \mathrm{g} / \mathrm{L}$ does not reduce the risk of cancer and in fact may even increase cancer risk [4, 13, 14].

We investigated toenail selenium levels of participants in the Netherlands Cohort Study. The study was conducted over a wide range of selenium that included low selenium. After a follow-up of 17.3 years, the analysis included 898 advanced PCa cases. The aim of the analysis was to investigate whether higher toenail selenium levels are associated with a decreased risk of advanced PCa.

\section{Methods}

\section{Study population}

The Netherlands Cohort Study included 58,279 men, aged 55 to 69 years at baseline in September 1986. All cohort members completed a baseline questionnaire and $79 \%$ of participants provided toenail clippings from all toes, which were used for selenium measurements [15]. Cancer cases were identified by annual record linkage to the Netherlands Cancer Registry and the Dutch Pathology Registry [16, 17]. The Netherlands Cohort Study has a case-cohort design; case subjects are derived from the entire cohort and the person-time experience is estimated from a subcohort randomly sampled from the full cohort at baseline $(n=1,688)$ [18]. In our analysis, we excluded all cases with prevalent cancer other than skin cancer at baseline and men with incomplete or inconsistent dietary questionnaire data $[19,20]$. The Netherlands Cohort Study has been approved by the institutional review boards of the TNO Nutrition and Food Research Institute and Maastricht University.

\section{Ascertainment and classification of prostate cancer cases}

During 17.3 years of follow-up, 3,451 incident PCa cases were identified. In the present analysis, we evaluated advanced PCa only (International Union Against Cancer (UICC) [21] stage III/IV), which was defined as T3-4, N+, or M1 at diagnosis ( $n=1,196)$. Stage IV (UICC) PCa is a subset of advanced PCa and was defined as stage T4, N+, or M1 at diagnosis ( $n=753$ ). A number of cases had missing data on tumor stage ( $n=216$ ). Baseline characteristics (e.g. age, smoking, and PCa family history) were not different between cases with and without data on tumor stage.

\section{Exposure assessment}

Selenium concentrations were measured in toenail clippings by the Reactor Institute Delft, using instrumental neutron activation analysis of the ${ }^{77 \mathrm{~m}} \mathrm{Se}$ isotope. Each sample went through six cycles of 17 -second irradiation at a thermal neutron flux of $3 \times 10^{16}$ $\mathrm{m}^{-2} \mathrm{~s}^{-1}, 3$-second decay, and 17 -second counting at $1 \mathrm{~cm}$ from a $40 \%$ germanium detector. The accuracy of the analysis was checked by a certified bovine liver standard 
(Standard Reference Material 1577b of the US National Institute of Standards and Technology). This method and the Netherlands Cohort Study toenail selenium project have been described in more detail previously [22-24].

Toenail selenium measurements for the subcohort were carried out in 1992. In 2002, toenail selenium levels of advanced PCa cases diagnosed during the first 6.3 years of follow-up were determined [8]. In 2011, toenail selenium levels of advanced PCa cases diagnosed during 6.3 to 17.3 years of follow-up were measured. In 1992, the 'Snelle Buizen Post' (SBP) facility was used for instrumental neutron activation analysis, and, since 1996, the 'Carbonfiber Autonomous Facility for Irradiation and Analysis' (CAFIA) facility is used. To assess the validity and comparability of these two methods, toenail selenium levels of the same 40 subcohort members were determined in 1996 with the 'CAFIA' facility in addition to the original assessment with the 'SBP' facility [25]. The mean selenium level ( $\mu \mathrm{g} / \mathrm{g}$ ) assessed by the 'CAFIA' facility $(0.552$; standard deviation (SD): 0.05$)$ was similar to the mean selenium level $(\mu \mathrm{g} / \mathrm{g}$ ) assessed by the 'SBP' facility (0.551; SD: 0.04), with a Pearson correlation coefficient of $0.95(P<0.01)$ [25]. It was concluded that both methods were valid and comparable.

Available toenail material from all toes was used for the selenium determination. We excluded participants who did not provide a toenail sample or had a sample with a too low sample weight (<10 mg). Baseline characteristics (e.g. age, smoking, and PCa family history) were not different between men with and without toenail selenium data. Complete toenail selenium data were available for 898 advanced PCa cases and 1,176 subcohort members.

\section{Statistical analysis}

We compared average toenail selenium levels of cases diagnosed in different years of follow-up to evaluate to potential influence of prediagnostic cancer at baseline on toenail selenium levels. Cox proportional hazards regression was used to obtain hazard ratios (HRs) and 95\% confidence intervals ( $\mathrm{Cls}$ ) for the association between quintiles of toenail selenium and advanced PCa risk. Standard errors were estimated by using the robust Huber-White sandwich estimator, to account for additional variance introduced by sampling from the cohort $[18,26]$. Cut-off points for each quintile were based on the distribution of toenail selenium in the subcohort. P-values for linear trends were calculated by using median values within toenail selenium quintiles. For the continuous analysis we used an increment of $0.045 \mu \mathrm{g} / \mathrm{g}$ of toenail selenium, which is the width of the central quintile. We completed age-adjusted and multivariable models adjusted for age, first-degree family history of $\mathrm{PCa}$, smoking status, duration of smoking, and frequency of smoking. We adjusted for cigarette smoking because it has been shown to be an important predictor of toenail selenium status $[5,23]$. The proportional hazards assumption was tested using the scaled Schoenfeld residuals [27], and we 
found no violation of the assumption. Time on study (follow-up) was used as time scale. Results were unchanged when age instead of time on study was used as time scale.

Several other variables were considered as potential confounders including: nonoccupational physical activity, education level, body mass index, history of diabetes, long-term use of non-steroidal anti-inflammatory drugs, black tea consumption, and intake of energy, alcohol, calcium, catechin, lycopene, red meat, and vitamin E. None of these was included in the final models, as they had little effect on the effect estimates or precision. We studied whether the association of toenail selenium and advanced PCa risk differed according to follow-up time by stratifying the follow-up time into two periods: $<8$ and $\geq 8$ years. Effect modification of the association between quantiles of toenail selenium and advanced PCa risk by other covariables was tested by using cross-product terms in the regression models and by examining stratum-specific HR-estimates. The Wald statistic was used to test for interaction. The following variables were considered as potential effect modifiers: first-degree family history of PCa, smoking status, body mass index, black tea consumption, and dietary antioxidant score. The antioxidant score is a measure of combined intake of $\beta$-carotene, catechin, lycopene, vitamin $C$, and vitamin $E$ (sum of quartile scores that range from 0 (low intake) to 3 (high intake)) and it has been described in more detail previously [28]. All tests were two-sided, with a P-value of less than 0.05 considered to be statistically significant. Analyses were performed using STATA software (release 12).

\section{Results}

Cases and subcohort members had an average toenail selenium level $(\mu \mathrm{g} / \mathrm{g})$ of 0.527 and 0.550 , respectively. We evaluated mean toenail selenium levels of cases diagnosed in different years of follow-up and we found no trend towards lower or higher levels indicating that there was no effect of preclinical disease (Table 2.1). Compared with subcohort members, cases were more likely to have a first-degree relative affected with PCa and less likely to have a history of diabetes (Table 2.2).

Toenail selenium was associated with a decreased risk of advanced (stage III/IV) PCa. Adjusted HRs (95\% Cls) for increasing quintiles of toenail selenium were 1.00 (reference), $0.75(0.57,1.00), 0.59(0.44,0.79), 0.43(0.31,0.58)$, and $0.37(0.27,0.51)$ ( $P$ for trend $<0.001$ ) (Table 3 ). The association was slightly more pronounced for stage IV PCa with an adjusted HR for the highest versus lowest quintile of toenail selenium of 0.30 (95\% Cl: 0.21, 0.45) (Table 2.3).

Table 2.4 shows the association between quintiles of toenail selenium and risk of advanced PCa stratified by period of follow-up. Adjusted HRs (95\% Cls) of advanced PCa (highest versus lowest quintile) for $<8$ and $\geq 8$ years of follow-up were 0.47 ( 0.30 , 
$0.76)$ and $0.31(0.21,0.45)$, respectively. The different outcome was also observed for stage IV PCa. The average age at diagnosis (SD) for advanced PCa cases who were diagnosed $<8$ and $\geq 8$ years of follow-up was 68.5 (4.2) and 74.5 years (4.7), respectively. The percentage of first-degree family history of PCa was higher for men who were diagnosed $<8$ years of follow-up (5.3\%) compared to $\geq 8$ years of follow up (2.8\%). Other characteristics were not different for these case groups.

There was no statistically significant interaction for any of the covariables tested for advanced PCa (Table 2.5). However, the association of tertiles of toenail selenium with advanced PCa appeared less pronounced for men with a first-degree family history of PCa ( $P$ for interaction $=0.504$ ). Furthermore, in stratified analysis, toenail selenium was associated with a reduced risk of advanced PCa among former smokers and current smokers, but not among never smokers ( $P$ for interaction $=0.306$ ).

Table 2.1

Mean toenail selenium level $(\mu \mathrm{g} / \mathrm{g})$ and standard deviation of men with advanced prostate cancer, per year of follow-up, Netherlands Cohort Study, 1986-2003 ${ }^{\mathrm{a}}$

\begin{tabular}{|c|c|c|c|}
\hline \multirow[b]{2}{*}{ Cases $^{b}$} & \multirow[b]{2}{*}{ No. cases } & \multicolumn{2}{|c|}{ Toenail selenium level ( $\mu \mathrm{g} / \mathrm{g})$} \\
\hline & & Mean & SD \\
\hline All cases & 898 & 0.527 & 0.169 \\
\hline \multicolumn{4}{|l|}{ Year of follow-up } \\
\hline 1 & 21 & 0.524 & 0.060 \\
\hline 2 & 17 & 0.530 & 0.077 \\
\hline 3 & 23 & 0.531 & 0.106 \\
\hline 4 & 35 & 0.538 & 0.090 \\
\hline 5 & 36 & 0.505 & 0.094 \\
\hline 6 & 55 & 0.542 & 0.090 \\
\hline 7 & 57 & 0.508 & 0.092 \\
\hline 8 & 76 & 0.555 & $0.428^{c}$ \\
\hline 9 & 68 & 0.526 & 0.150 \\
\hline 10 & 77 & 0.511 & 0.065 \\
\hline 11 & 77 & 0.553 & 0.238 \\
\hline 12 & 65 & 0.523 & 0.082 \\
\hline 13 & 54 & 0.539 & 0.146 \\
\hline 14 & 54 & 0.517 & 0.078 \\
\hline 15 & 56 & 0.538 & 0.122 \\
\hline 16 & 56 & 0.524 & 0.097 \\
\hline 17 & 56 & 0.500 & 0.070 \\
\hline 18 & 15 & 0.471 & 0.053 \\
\hline
\end{tabular}

Abbreviation: SD, standard deviation.

${ }^{a}$ Advanced (stage III/IV) prostate cancers were stage T3-4, $\mathrm{N}+$, or $\mathrm{M} 1$ at diagnosis.

${ }^{b}$ Mean toenail selenium level (SD) of subcohort members was $0.550 \mu \mathrm{g} / \mathrm{g}(0.129)$.

${ }^{\mathrm{c}}$ The large SD is the result of an extreme, but biologically plausible, observation $(4.174 \mu \mathrm{g} / \mathrm{g})$. 
Table 2.2

Characteristics of advanced prostate cancer cases and subcohort members, Netherlands Cohort Study, 1986-2003

\begin{tabular}{|c|c|c|c|c|}
\hline \multirow[b]{2}{*}{ Variables } & \multicolumn{2}{|c|}{$\begin{array}{l}\text { Advanced prostate } \\
\text { cancer cases }{ }^{a} \\
(n=898)\end{array}$} & \multicolumn{2}{|c|}{$\begin{array}{l}\text { Subcohort members } \\
(n=1,176)\end{array}$} \\
\hline & $\%$ & Mean (SD) & $\%$ & Mean (SD) \\
\hline Age at baseline, years & & $62.1(4.1)$ & & $61.3(4.2)$ \\
\hline Age at diagnosis, years & & $72.3(5.3)$ & & - \\
\hline First-degree family history of prostate cancer & 3.7 & & 2.6 & \\
\hline \multicolumn{5}{|l|}{ Smoking status } \\
\hline Never & 13.9 & & 13.8 & \\
\hline Former & 56.1 & & 53.3 & \\
\hline Current & 30.0 & & 32.9 & \\
\hline Duration of smoking ${ }^{\dagger}$, years & & $32.5(12.2)$ & & $32.8(12.0)$ \\
\hline Frequency of smoking, cigarettes $/$ day $^{b}$ & & $16.1(10.4)$ & & $16.9(10.5)$ \\
\hline $\begin{array}{l}\text { High (>90 minutes/day) non-occupational } \\
\text { physical activity }\end{array}$ & 33.5 & & 31.9 & \\
\hline Higher vocational or university education & 21.3 & & 18.0 & \\
\hline Body mass index, $\mathrm{kg} / \mathrm{m}^{2}$ & & $25.0(2.5)$ & & $25.0(2.7)$ \\
\hline History of diabetes & 2.2 & & 3.3 & \\
\hline Long-term use of NSAIDs ${ }^{c}$ & 5.0 & & 6.5 & \\
\hline Black tea consumption, cups/day & & $2.6(2.0)$ & & $2.6(2.0)$ \\
\hline \multicolumn{5}{|l|}{ Dietary intake } \\
\hline Energy, kcal/day & & $2,157(480)$ & & $2,160(505)$ \\
\hline Alcohol, g/day & & $14.5(15.5)$ & & $14.5(16.7)$ \\
\hline Calcium, g/day & & $956(322)$ & & $942(332)$ \\
\hline Catechin, mg/day & & $58.2(38.0)$ & & $57.8(37.0)$ \\
\hline Lycopene, mg/day & & $1.1(1.6)$ & & $1.0(1.6)$ \\
\hline Red meat, g/day & & $91.9(39.6)$ & & $93.0(41.6)$ \\
\hline Vitamin E, mg/day & & $15.2(6.7)$ & & $14.8(6.7)$ \\
\hline$\beta$-carotene, mg/day & & $2.9(1.4)$ & & $3.0(1.6)$ \\
\hline Vitamin C, mg/day & & 101.2 (41.9) & & $98.8(40.9)$ \\
\hline
\end{tabular}

Abbreviations: SD, standard deviation; NSAIDs, non-steroidal anti-inflammatory drugs.

The variable 'Age at diagnosis' does not apply to subcohort members.

${ }^{a}$ Advanced (stage III/IV) prostate cancers were stage T3-4, $\mathrm{N}+$, or $\mathrm{M} 1$ at diagnosis.

${ }^{b}$ Never smokers were excluded.

${ }^{\mathrm{c}}$ Use of aspirin or other NSAIDs for a period of at least six months. 


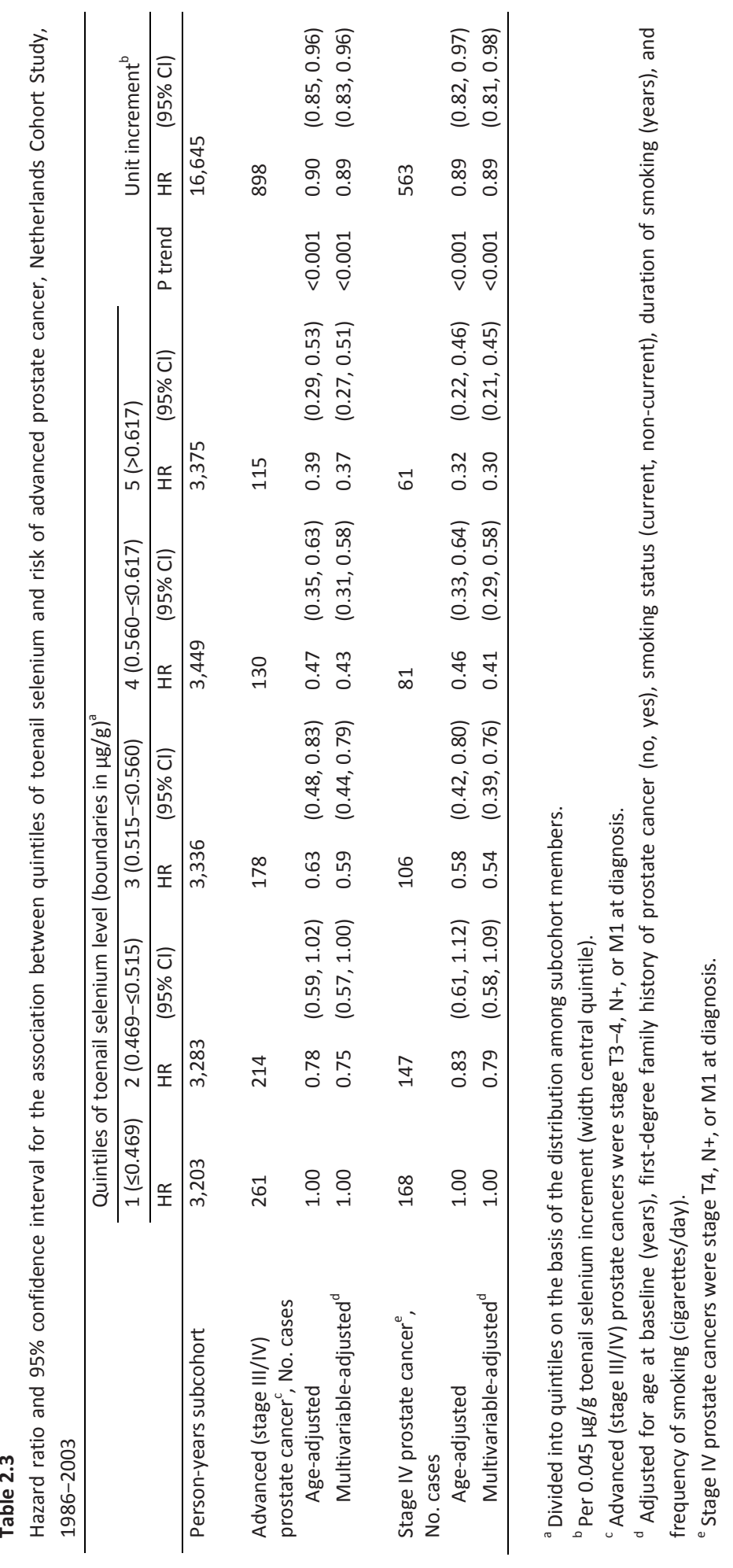




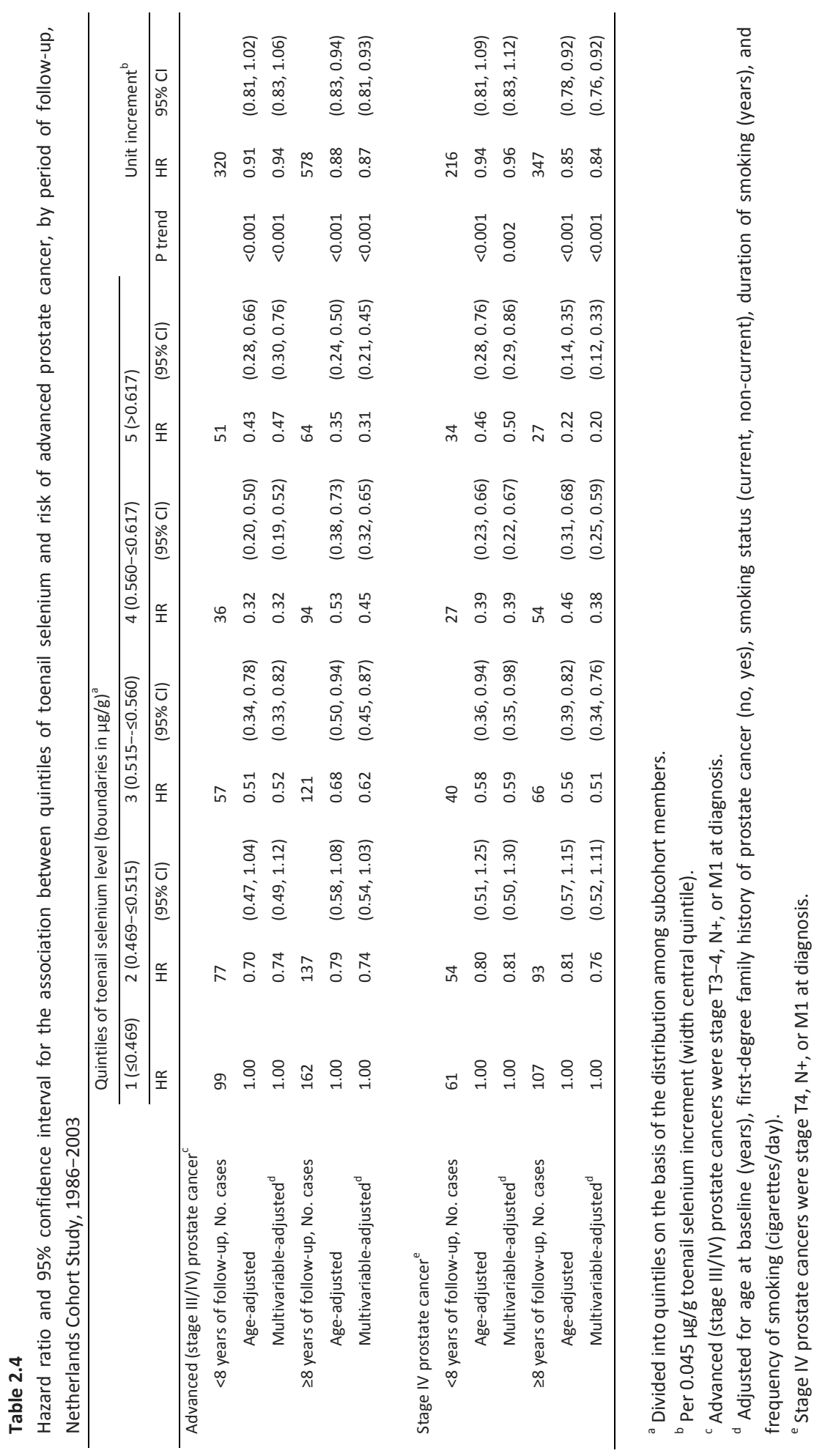




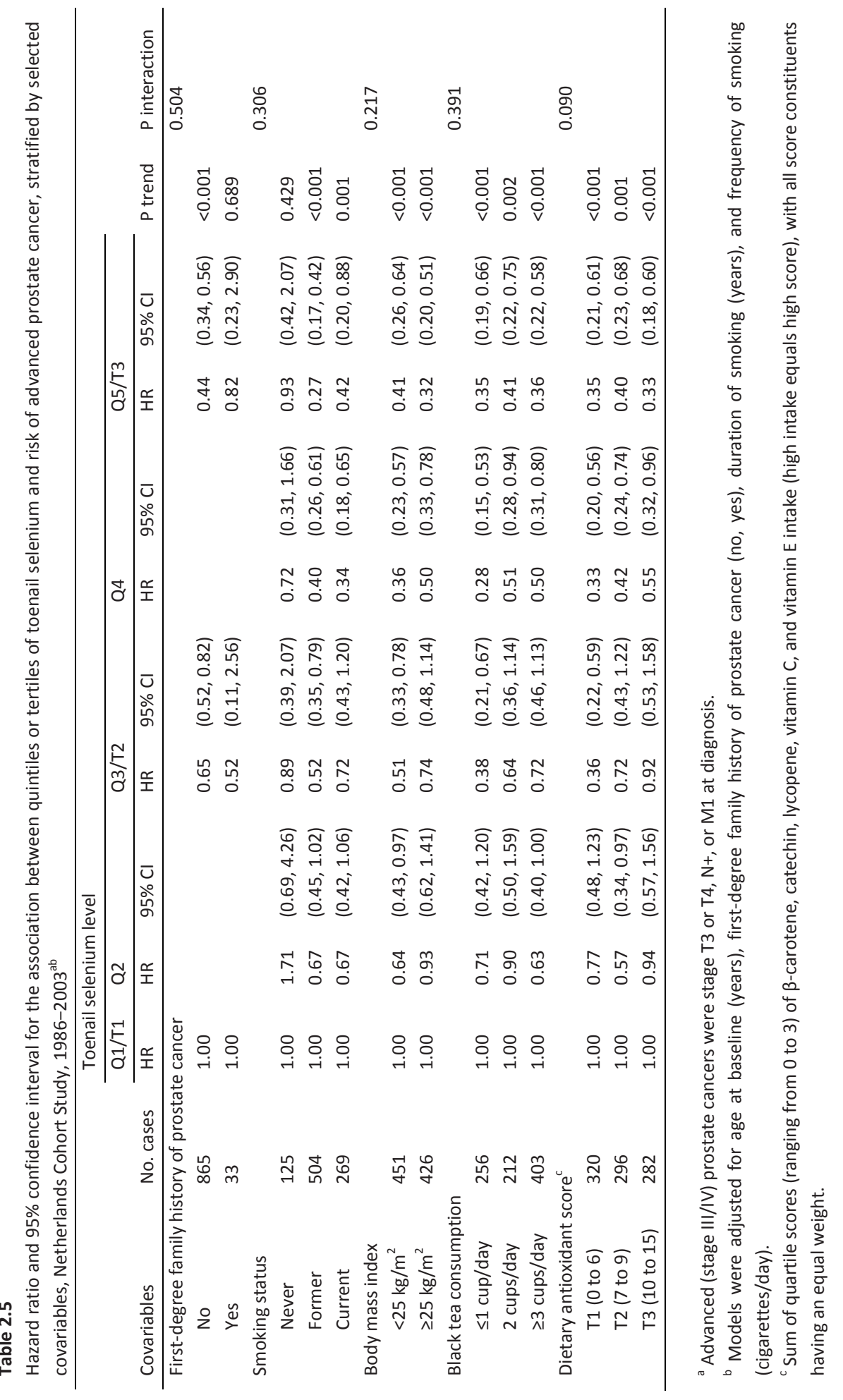




\section{Discussion}

This prospective long-term follow-up study in the Netherlands showed that toenail selenium was associated with a decreased risk of advanced PCa, with men in the highest compared to the lowest quintile of toenail selenium having a $63 \%$ reduced risk.

Strengths of our study include the prospective design, population-based approach, long and nearly complete follow-up of the study population through linkage to cancer registries, and detailed data on potential confounders. We specifically evaluated advanced PCa and our study included a large number of these cases. Our study was conducted over a wide range of selenium that included low selenium. We used toenail selenium for exposure monitoring, which reflects long-term selenium intake. The study has some limitations as well. Selenium levels of study participants were determined at different points in time and this may have introduced bias. To avoid bias by analyzing stored toenail samples at different time points, the same standard reference material was used to check the accuracy of the neutron activation analyses over time. Another limitation is that we only measured selenium at baseline and had no repeated exposure measurements.

There have been three previous prospective analyses of toenail selenium and PCa risk. One of these studies was an earlier analysis in the Netherlands Cohort study [8]. The analysis included cases who were diagnosed during 6.3 years of follow-up and the relative risks of overall and advanced PCa were assessed (540 PCa cases; 183 advanced cases). The study showed that men in the highest versus lowest quintile of toenail selenium had a decreased risk of overall PCa ( $\mathrm{HR}=0.69 ; 95 \% \mathrm{Cl}: 0.48,0.99)$ and advanced PCa (HR $=0.62 ; 95 \% \mathrm{Cl}: 0.37,1.05)$. An US-based study by Helzlsouer et al. (117 cases and 233 controls) showed an HR of overall PCa of 0.38 (95\% Cl: $0.17,0.85$ ) for men in the highest versus lowest quintile of toenail selenium [29]. Advanced PCa risk was not assessed in that study. Mean toenail selenium was higher in the study by Helzlsouer et al. compared to the Netherlands Cohort Study (quintile medians $(\mu \mathrm{g} / \mathrm{g}$ ) ranging from $0.66-0.96$ and $0.43-0.67$, respectively). In another US-based study of toenail selenium, Yoshizawa et al. specifically evaluated advanced PCa [30]. The study included 181 advanced PCa cases and 181 controls and toenail selenium quintile medians ranged from $0.66-1.14 \mu \mathrm{g} / \mathrm{g}$. The authors reported an adjusted odds ratio of 0.35 ( $95 \% \mathrm{Cl}: 0.16,0.78)$ for men in the highest compared to the lowest quintile of toenail selenium.

Most observational studies of selenium and PCa have used plasma/serum rather than toenails for exposure monitoring [6]. Data of these studies have been pooled in a recent meta-analysis that evaluated both overall PCa (nine studies) and advanced PCa (six studies) [6]. For overall PCa, the authors found a gradual reduction in risk over the plasma/serum selenium range investigated (60-170 $\mu \mathrm{g} / \mathrm{L})$, with an estimated relative 
risk $(95 \% \mathrm{Cl})$ at 135 and $170 \mu \mathrm{g} / \mathrm{L}$ of $0.85(0.74,0.97)$ and $0.75(0.65,0.86)$, respectively. The association was more pronounced for advanced PCa (same range of selenium) with an estimated relative risk $(95 \% \mathrm{Cl})$ at 135 and $170 \mu \mathrm{g} / \mathrm{L}$ of $0.60(0.45$, $0.81)$ and $0.50(0.36,0.68)$, respectively. Most of the pooled studies were conducted in the US where selenium intake is often high.

Our results further support that selenium intake may be an important modifiable factor that reduces the risk of advanced PCa. We specifically evaluated advanced PCa, because these cancers have a poor prognosis and are, therefore, clinically relevant. Several studies have provided evidence that the relationship between selenium and cancer may be U-shaped, with a higher risk associated with levels both below and above an optimal selenium range [4, 31]. The Nutritional Prevention of Cancer (NPC) trial showed that selenium supplementation was associated with a reduced risk of total PCa risk. The association, however, was only present among participants with baseline plasma selenium concentrations in the lowest two tertiles; those in the highest tertile (>122 $\mathrm{\mu g} / \mathrm{L})$ showed a non-significant increase in risk [13, 14]. In our study, quintile medians of toenail selenium ranged from 0.43 to $0.67 \mu \mathrm{g} / \mathrm{g}$. This range of toenail selenium was estimated to be equivalent to (quintile medians) 64-100 $\mu \mathrm{g} / \mathrm{L}$ plasma selenium by using the method described by Waters et al. [31]. These estimated levels are comparable to observed plasma selenium levels reported in other epidemiological studies in the Netherlands [32-35]. Based on results of the NPC trial, selenium levels in our study are in the range where additional selenium is expected to be beneficial, in the proposed U-shaped curve [4, 31]. In addition, we observed that the association of toenail selenium and advanced PCa risk was slightly more pronounced for men diagnosed during later follow-up at an older age. Prostate cancer diagnosed at a younger age is more often related with a positive family history of PCa, and it may be that selenium is less effective for preventing this type of $\mathrm{PCa}$, which may be fundamentally different from sporadic PCa $[36,37]$. Most prior prospective studies of selenium and PCa had a follow-up time that was considerably shorter than in our study and did not include results for separate follow-up periods. Further large and long-term follow-up studies are therefore urgently needed.

Most of our study participants were smokers (either former or current), with only $14 \%$ of men having never smoked. A number of studies showed evidence that the inverse association of selenium and PCa risk is particularly evident among smokers [8, 38-40]. This finding is biologically plausible given that selenium is an antioxidant and smoking results in increased oxidative stress [2]. In a stratified analysis, we showed that toenail selenium was inversely associated with advanced PCa risk among smokers, but not among never smokers, with no significant interaction. The analysis included only 125 cases who were never smokers, suggesting that power may have been too low. 
Prospective studies showing evidence of an association between selenium and reduced PCa risk are not supported by SELECT [10]. A number of possible explanations for these different findings have been suggested. In particular, it may be that baseline selenium levels in SELECT were too high to have an effect of further selenium supplementation on PCa risk [4, 11, 12]. The interquartile range of baseline serum selenium in SELECT was $122-152 \mu \mathrm{g} / \mathrm{L}$ and prior evidence showed that subjects in this range of baseline selenium have no benefit from selenium supplementation $[4,13,14]$. It would, therefore, be of great interest to see SELECT results stratified by baseline selenium levels. Several observational studies showed a greater effect of selenium on advanced versus non-advanced PCa. Because of prostate-specific antigen (PSA) screening practices in the US, in SELECT, PCa was most often diagnosed at localized stages; only $1 \%$ of men diagnosed with PCa in SELECT had stage T3 at diagnosis [10]. In comparison, in the Netherlands Cohort Study, where PSA screening is less frequent, $35 \%$ of incident PCa was advanced PCa.

In conclusion, our large prospective analysis showed that higher toenail selenium concentrations were associated with a substantial decrease in risk of advanced PCa. If our results are confirmed, a prevention trial of selenium and PCa in a low-selenium population may be justified.

\section{References}

1. Papp LV, Lu J, Holmgren A, et al. From selenium to selenoproteins: synthesis, identity, and their role in human health. Antioxid Redox Signal 2007;9(7):775-806.

2. Peters U, Takata Y. Selenium and the prevention of prostate and colorectal cancer. Mol Nutr Food Res 2008;52(11):1261-1272.

3. Rayman MP. The importance of selenium to human health. Lancet 2000;356(9225):233-241.

4. Rayman MP. Selenium and human health. Lancet 2012;379(9822):1256-1268.

5. Park K, Rimm E, Siscovick D, et al. Demographic and lifestyle factors and selenium levels in men and women in the U.S. Nutr Res Pract 2011;5(4):357-364.

6. Hurst R, Hooper L, Norat $T$, et al. Selenium and prostate cancer: systematic review and meta-analysis. Am J Clin Nutr 2012;96(1):111-122.

7. Slotnick MJ, Nriagu JO. Validity of human nails as a biomarker of arsenic and selenium exposure: $A$ review. Environ Res 2006;102(1):125-139.

8. van den Brandt PA, Zeegers MP, Bode P, et al. Toenail selenium levels and the subsequent risk of prostate cancer: a prospective cohort study. Cancer Epidemiol Biomarkers Prev 2003;12(9):866-871.

9. Lippman SM, Klein EA, Goodman PJ, et al. Effect of selenium and vitamin E on risk of prostate cancer and other cancers: the Selenium and Vitamin E Cancer Prevention Trial (SELECT). Jama 2009;301(1):39-51.

10. Klein EA, Thompson IM, Jr., Tangen CM, et al. Vitamin E and the risk of prostate cancer: the Selenium and Vitamin E Cancer Prevention Trial (SELECT). Jama 2011;306(14):1549-1556.

11. Dunn BK, Ryan A, Ford LG. Selenium and Vitamin E Cancer Prevention Trial: a nutrient approach to prostate cancer prevention. Recent Results Cancer Res 2009;181:183-193.

12. Schrauzer GN. RE: Lessons from the selenium and vitamin E cancer prevention trial (SELECT). Crit Rev Biotechnol 2009;29(2):81.

13. Duffield-Lillico AJ, Reid ME, Turnbull BW, et al. Baseline characteristics and the effect of selenium supplementation on cancer incidence in a randomized clinical trial: a summary report of the Nutritional Prevention of Cancer Trial. Cancer Epidemiol Biomarkers Prev 2002;11(7):630-639. 
14. Duffield-Lillico AJ, Dalkin BL, Reid ME, et al. Selenium supplementation, baseline plasma selenium status and incidence of prostate cancer: an analysis of the complete treatment period of the Nutritional Prevention of Cancer Trial. BJU Int 2003;91(7):608-612.

15. van den Brandt PA, Goldbohm RA, van 't Veer $\mathrm{P}$, et al. A large-scale prospective cohort study on diet and cancer in The Netherlands. J Clin Epidemiol 1990;43(3):285-295.

16. van den Brandt PA, Schouten LJ, Goldbohm RA, et al. Development of a record linkage protocol for use in the Dutch Cancer Registry for epidemiological research. Int J Epidemiol 1990;19(3):553-558.

17. Goldbohm RA, van den Brandt PA, Dorant E. Estimation of the coverage of Dutch municipalities by cancer registries and PALGA based on hospital discharge data. Tijdschr Soc Gezondheidsz. $1994 ; 19(3): 80-84$.

18. Barlow WE, Ichikawa L, Rosner D, et al. Analysis of case-cohort designs. J Clin Epidemiol 1999;52(12):1165-1172.

19. Goldbohm RA, van den Brandt PA, Brants HA, et al. Validation of a dietary questionnaire used in a largescale prospective cohort study on diet and cancer. Eur J Clin Nutr 1994;48(4):253-265.

20. Goldbohm RA, van 't Veer P, van den Brandt PA, et al. Reproducibility of a food frequency questionnaire and stability of dietary habits determined from five annually repeated measurements. Eur J Clin Nutr 1995;49(6):420-429.

21. Sobin L, Gospodarowicz M, Wittekind C, et al. TNM Classification of Malignant Tumors. 7th ed. Oxford, UK: Wiley-Blackwell; 2009.

22. van den Brandt PA, Goldbohm RA, van 't Veer $P$, et al. A prospective cohort study on toenail selenium levels and risk of gastrointestinal cancer. J Natl Cancer Inst 1993;85(3):224-229.

23. van den Brandt PA, Goldbohm RA, van't Veer $P$, et al. Predictors of toenail selenium levels in men and women. Cancer Epidemiol Biomarkers Prev 1993;2(2):107-112.

24. Steevens J, van den Brandt PA, Goldbohm RA, et al. Selenium status and the risk of esophageal and gastric cancer subtypes: the Netherlands cohort study. Gastroenterology 2010;138(5):1704-1713.

25. Zeegers MP, Goldbohm RA, Bode $P$, et al. Prediagnostic toenail selenium and risk of bladder cancer. Cancer Epidemiol Biomarkers Prev 2002;11(11):1292-1297.

26. Lin DY, Wei LY. The robust inference for the Cox proportional hazards model. J Am Stat Assoc. 1989;84:1074-1078.

27. Grambsch PM, Therneau TM. Proportional hazards tests and diagnostics based on weighted residuals. Biometrika 1994;81(3):515-526.

28. Geybels MS, Verhage BA, van Schooten FJ, et al. Measures of combined antioxidant and pro-oxidant exposures and risk of overall and advanced stage prostate cancer. Ann Epidemiol 2012;22(11):814-820.

29. Helzlsouer KJ, Huang HY, Alberg AJ, et al. Association between alpha-tocopherol, gamma-tocopherol, selenium, and subsequent prostate cancer. J Natl Cancer Inst 2000;92(24):2018-2023.

30. Yoshizawa K, Willett WC, Morris SJ, et al. Study of prediagnostic selenium level in toenails and the risk of advanced prostate cancer. J Natl Cancer Inst 1998;90(16):1219-1224.

31. Waters DJ, Shen S, Glickman LT, et al. Prostate cancer risk and DNA damage: translational significance of selenium supplementation in a canine model. Carcinogenesis 2005;26(7):1256-1262.

32. Allen NE, Appleby PN, Roddam AW, et al. Plasma selenium concentration and prostate cancer risk: results from the European Prospective Investigation into Cancer and Nutrition (EPIC). Am J Clin Nutr 2008;88(6):1567-1575.

33. Kok FJ, Hofman A, Witteman JC, et al. Decreased selenium levels in acute myocardial infarction. Jama 1989;261(8):1161-1164.

34. Bukkens SG, de Vos N, Kok FJ, et al. Selenium status and cardiovascular risk factors in healthy Dutch subjects. J Am Coll Nutr 1990;9(2):128-135

35. van 't Veer $P$, van der Wielen RP, Kok FJ, et al. Selenium in diet, blood, and toenails in relation to breast cancer: a case-control study. Am J Epidemiol 1990;131(6):987-994.

36. Bratt O. Hereditary prostate cancer: clinical aspects. J Urol 2002;168(3):906-913.

37. Verhage BA, Kiemeney LA. Inherited predisposition to prostate cancer. Eur J Epidemiol 2003;18(11):1027-1036.

38. Nomura AM, Lee J, Stemmermann GN, et al. Serum selenium and subsequent risk of prostate cancer. Cancer Epidemiol Biomarkers Prev 2000;9(9):883-887.

39. Vogt TM, Ziegler RG, Graubard BI, et al. Serum selenium and risk of prostate cancer in U.S. blacks and whites. Int J Cancer 2003;103(5):664-670. 
40. Peters U, Foster CB, Chatterjee N, et al. Serum selenium and risk of prostate cancer-a nested casecontrol study. Am J Clin Nutr 2007;85(1):209-217. 


\section{Chapter 3: Selenoprotein Gene Variants, Toenail Selenium Levels, and Risk of Advanced Prostate}

\section{Cancer}

Milan S. Geybels

Piet A. van den Brandt

Leo J. Schouten

Frederik J. van Schooten

Simone G. van Breda

Margaret P. Rayman

Fiona R. Green

Bas A.J. Verhage

Journal of the National Cancer Institute 2014;106(3):dju003 


\section{Abstract}

\section{Background}

Lower selenium levels have been associated with increased risk of prostate cancer (PCa), and genetic variation in the selenoprotein genes selenoprotein P (SEPP1) and glutathione peroxidase 1 (GPX1) is thought to modify this relationship.

\section{Methods}

We investigated whether the association between toenail selenium levels and advanced PCa risk in the prospective Netherlands Cohort Study is modified by common genetic variation in SEPP1 and GPX1. Toenail clippings were used to determine selenium levels and to isolate DNA for genotyping. This case-cohort study, including 817 advanced PCa cases and 1048 subcohort members, was analyzed with Cox regression models. All statistical tests were two-sided.

\section{Results}

Three genetic variants were associated with advanced (stage III/IV or IV) PCa risk: SEPP1 rs7579 ( $P$ for trend = 0.012), GPX1 rs17650792 ( $P$ for trend $=0.033$ ), and GPX1 rs1800668 ( $P$ for trend $=0.005$ ). Toenail selenium levels were inversely associated with advanced PCa risk, independently of common genetic variation in SEPP1 and GPX1.

\section{Conclusion}

Common genetic variants in SEPP1 and GPX1 were marginally associated with advanced PCa risk but did not modify the association between toenail selenium levels and advanced PCa risk. 


\section{Introduction}

The micronutrient selenium is a key component of selenoproteins that have important biological activities [1]. Lower selenium levels have repeatedly been associated with an increased PCa risk [2-4]. However, some large epidemiological studies [2], including the Selenium and Vitamin E Cancer Prevention Trial (SELECT) [5], do not support this inverse relationship. As a result of these disparate findings, it has been hypothesized that the effect of selenium on PCa is modified by selenoprotein gene variation [3, 6-8].

Plausible candidate selenoprotein genes to genetically modify the selenium-PCa association are selenoprotein P (SEPP1) and glutathione peroxidase 1 (GPX1). Genetic variants in both genes have previously been identified as having an effect on PCa risk $[7,9]$. Furthermore, these genes have known essential biological functions; SEPP1 is involved in selenium transport [10], and GPX1 counteracts oxidative stress [11].

We investigated common SEPP1 and GPX1 variation in relation to advanced PCa risk and examined potential interactions with selenium levels measured in toenail samples. In a previous report, based on the same study population, we showed that men in the highest versus lowest toenail selenium quintile had a more than 60 percent reduced risk of advanced $\mathrm{PCa}$ [12].

\section{Methods}

\section{Study population and design}

The Netherlands Cohort Study included 58,279 men, aged 55 to 69 years at baseline in 1986 [13]. Approximately $81 \%$ of participants provided toenail clippings, which were used to determine selenium levels and to extract DNA. Cancer cases were identified during 17.3 years of follow-up by annual record linkage to cancer registries [14]. Advanced PCa was defined as T3-4, N+, or M1 at diagnosis (International Union Against Cancer stage III/IV [15]) ( $n=1290)$. Stage IV PCa was defined as stage T4, N+, or M1 at diagnosis ( $\mathrm{n}=817$ ). The study had a case-cohort design and a flow diagram of study participants is shown in Figure 3.1. The study was approved by the institutional review boards of TNO and Maastricht University. Study participants gave informed consent. 


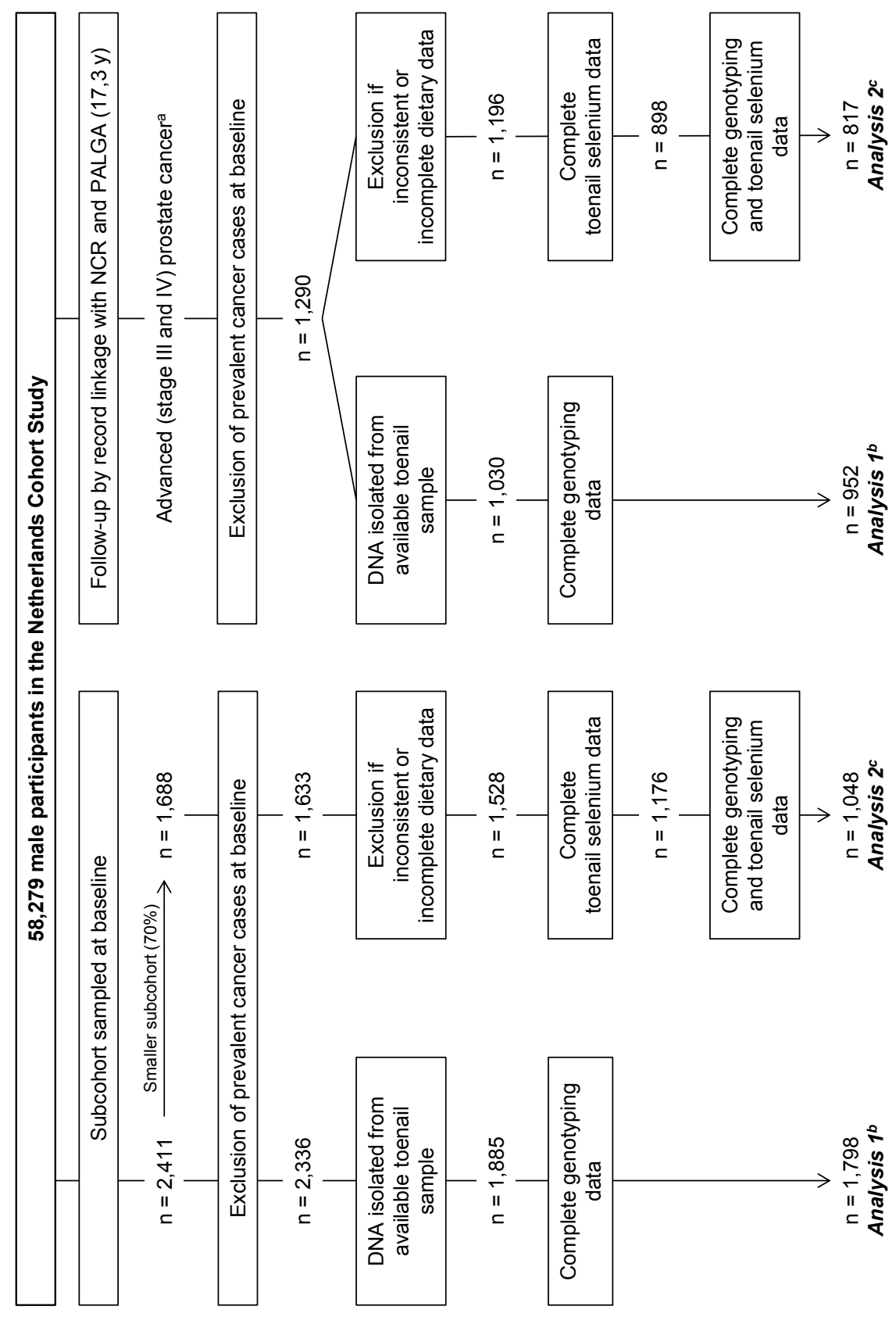




\section{Figure 3.1}

Flow diagram of cases and subcohort members for the analysis of genetic variants in selenoprotein $\mathrm{P}$ and glutathione peroxidase 1 , toenail selenium levels, and advanced prostate cancer risk, Netherlands Cohort Study, 1986-2003

Abbreviations: NCR, Netherlands Cancer Registry; PALGA, Dutch Pathology Registry.

${ }^{a}$ Advanced prostate cancer was defined as T3-4, N+, or M1 at diagnosis (International Union Against Cancer stage III/IV). On average, $63 \%$ of stage III/IV prostate cancer cases were stage IV prostate cancer (stage T4, $\mathrm{N}+$, or $\mathrm{M} 1$ at diagnosis).

${ }^{b}$ Analysis 1 was on SEPP1 and GPX1 genetic variation in relation to advanced prostate cancer risk and included 952 stage III/IV prostate cancer cases and 1,798 subcohort members. Samples with a sample call rate (i.e., percentage of called SNPs per sample) of less than $95 \%$ were excluded.

${ }^{c}$ Analysis 2 was on potential interactions between SEPP1 and GPX1 genetic variants and toenail selenium levels on advanced prostate cancer risk and included 817 stage III/IV prostate cancer cases and 1048 subcohort members. A smaller version of the subcohort (70\%) was used for this analysis.

\section{Selection of genetic variants and genotyping}

Tagging single nucleotide polymorphisms (tagSNPs) were identified utilizing the HapMap CEU population and parameters of $r^{2} \geq 0.8$ and minor allele frequency $\geq 10 \%$ [16]. SNPs were selected within genes and 5000 base pairs upstream and downstream. We identified five tagSNPs in SEPP1 and three in GPX1. DNA was extracted from toenails as described previously [17]. DNA was successfully isolated for 1,030 cases and 1,885 subcohort members (>99.9\% successful DNA isolation). Genotyping was done using MassARRAY software (v4.0) and the iPLEX Gold system (Sequenom Inc., Hamburg, Germany). Quality control included genotyping of blind duplicate samples, which revealed $>99 \%$ agreement on genotyping calls. Samples with a sample call rate of less than $95 \%$ were excluded and a total of 952 cases and 1,798 subcohort members had complete genotyping data. Most SNPs had $>99 \%$ genotype completion rates with the exception of rs230819 (85.7\%). None of the SNPs violated Hardy-Weinberg equilibrium $(P>0.05)$.

\section{Selenium concentrations}

Selenium concentrations were measured in toenails using instrumental neutron activation analysis [12]. For the gene-environment interaction analysis, we used a smaller subset of the subcohort (70\%) [18]; 817 cases and 1,048 subcohort members had complete genotyping and toenail selenium data.

\section{Statistical analyses}

Age-adjusted Cox proportional hazards regression models were used. The proportional hazards assumption was tested using the scaled Schoenfeld residuals [19], and we found no violation of the assumption. SNPs were analyzed under a co-dominant and log-additive genetic model. Standard errors were estimated using the robust HuberWhite sandwich estimator $[20,21]$. Multiplicative interactions of quartiles of toenail 
selenium and genotype (dominant model) were tested using cross-product terms and the Wald test. Selenium quartiles were based on the distribution in the subcohort. Interaction models were adjusted for age, first-degree family history of PCa, smoking status, and duration and frequency of smoking, as described previously [12]. Several other potential confounders were considered [12], but none of these were selected as they had little effect on the effect estimates $(<5 \%)$ or precision. All statistical tests were two-sided, with P-value $<0.05$ considered to be statistically significant. Analyses were performed using STATA software (Release 12, STATA Corporation, College Station, TX).

\section{Results}

Two GPX1 SNPs were associated with advanced (stage III/IV) PCa risk (Table 3.1). For GPX1 rs17650792, hazard ratios (HRs) for AA, AG, and GG were 1.00 (reference), 1.14 (95\% confidence interval $(\mathrm{Cl})=0.95,1.37)$, and $1.29(95 \% \mathrm{Cl}=1.01,1.63)$; $\mathrm{P}$ for trend $=$ 0.033. For GPX1 rs1800668, HRs for CC, CT, and TT were 1.00 (reference), 0.81 (95\% Cl $=0.68,0.96)$, and $0.73(95 \% \mathrm{Cl}=0.54,0.97) ; \mathrm{P}$ for trend $=0.005$. The HRs were similar for stage IV PCa. SEPP1 SNPs were not associated with stage III/IV PCa risk, but SEPP1 rs7579 was associated with stage IV PCa risk, with HRs for GG, AG, and AA of 1.00 (reference), $0.81(95 \% \mathrm{Cl}=0.66,0.99)$, and $0.71(95 \% \mathrm{Cl}=0.51,1.00) ; \mathrm{P}$ for trend $=$ 0.012 .

Higher toenail selenium levels were associated with a reduced risk of advanced PCa in all genotype subgroups (Table 3.2). None of the tested multiplicative interactions between genotype and selenium status were statistically significant. Results were comparable for stage IV PCa. When using only one reference category (i.e., men in the lowest selenium quartile with the common homozygote genotype), we observed evidence of a genetic effect of SEPP1 rs3877899, SEPP1 rs7579, and GPX1 rs3448 on advanced PCa risk among men in the lowest quartile of toenail selenium (Table 3.3). 


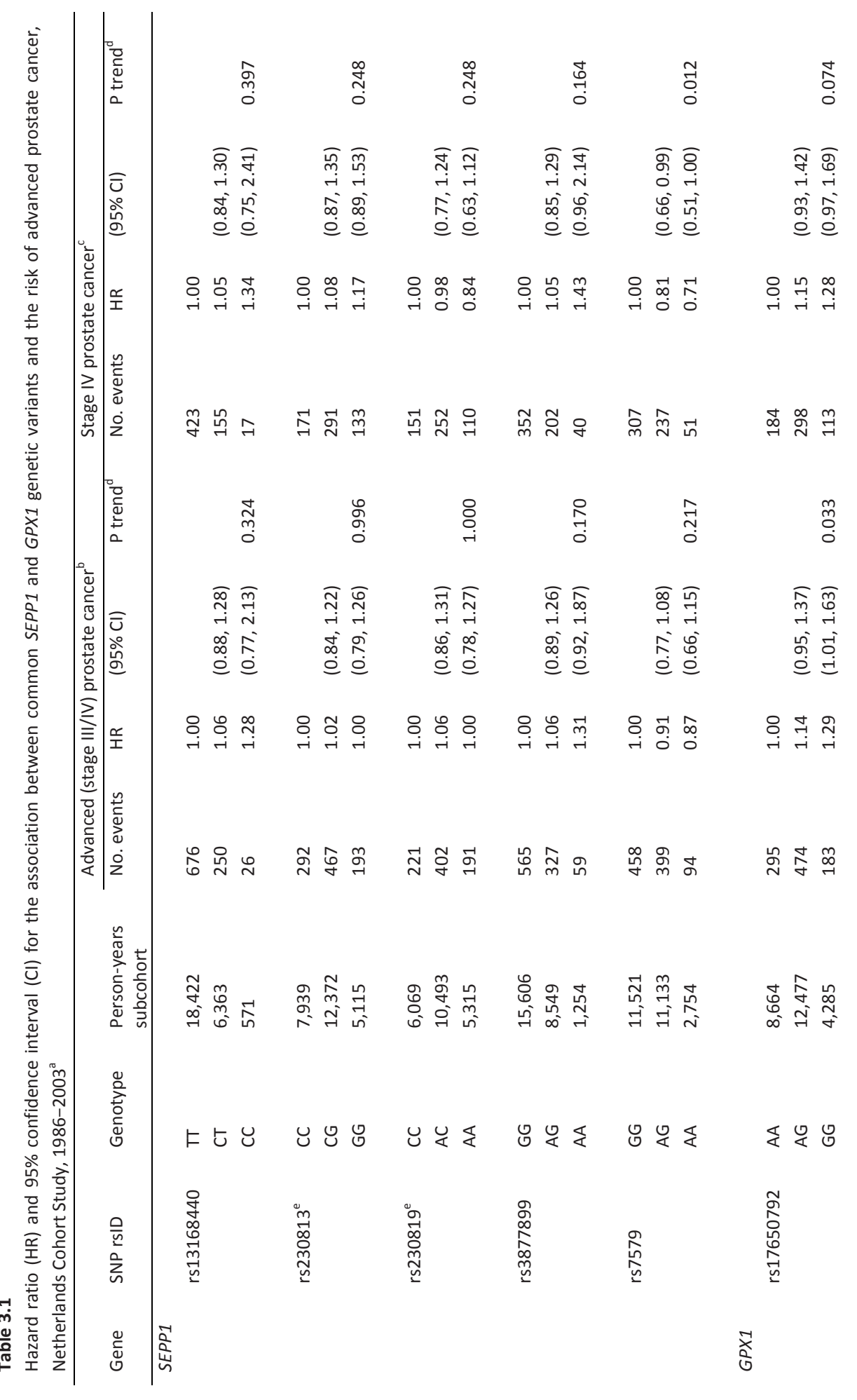




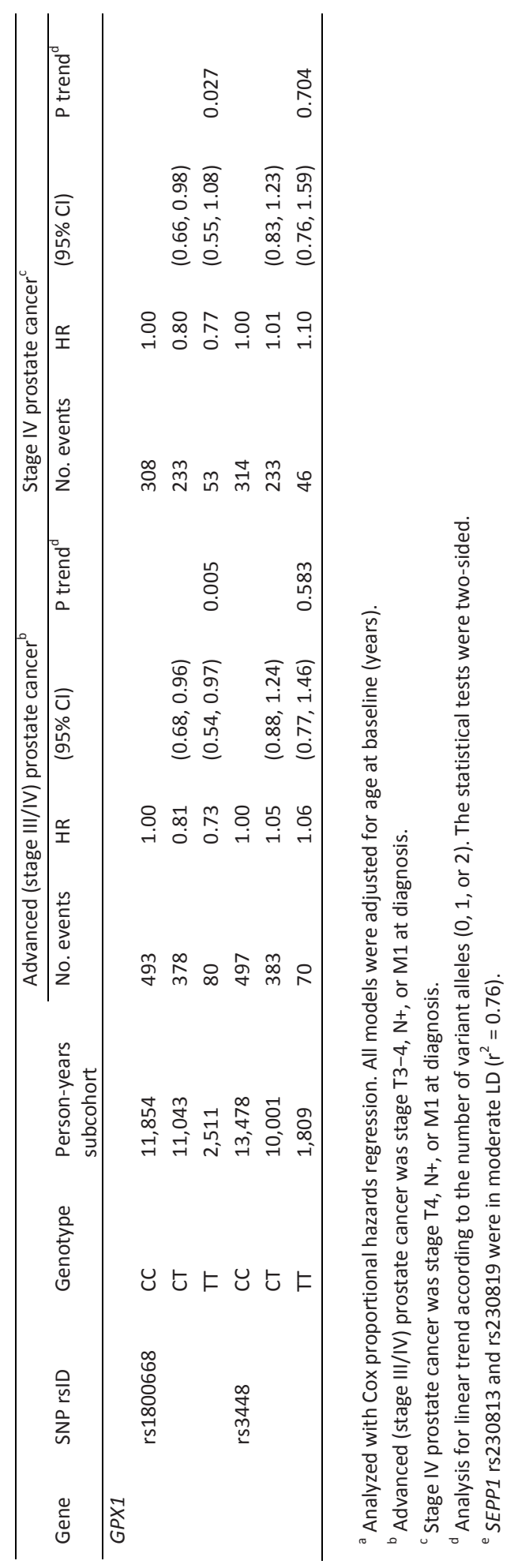




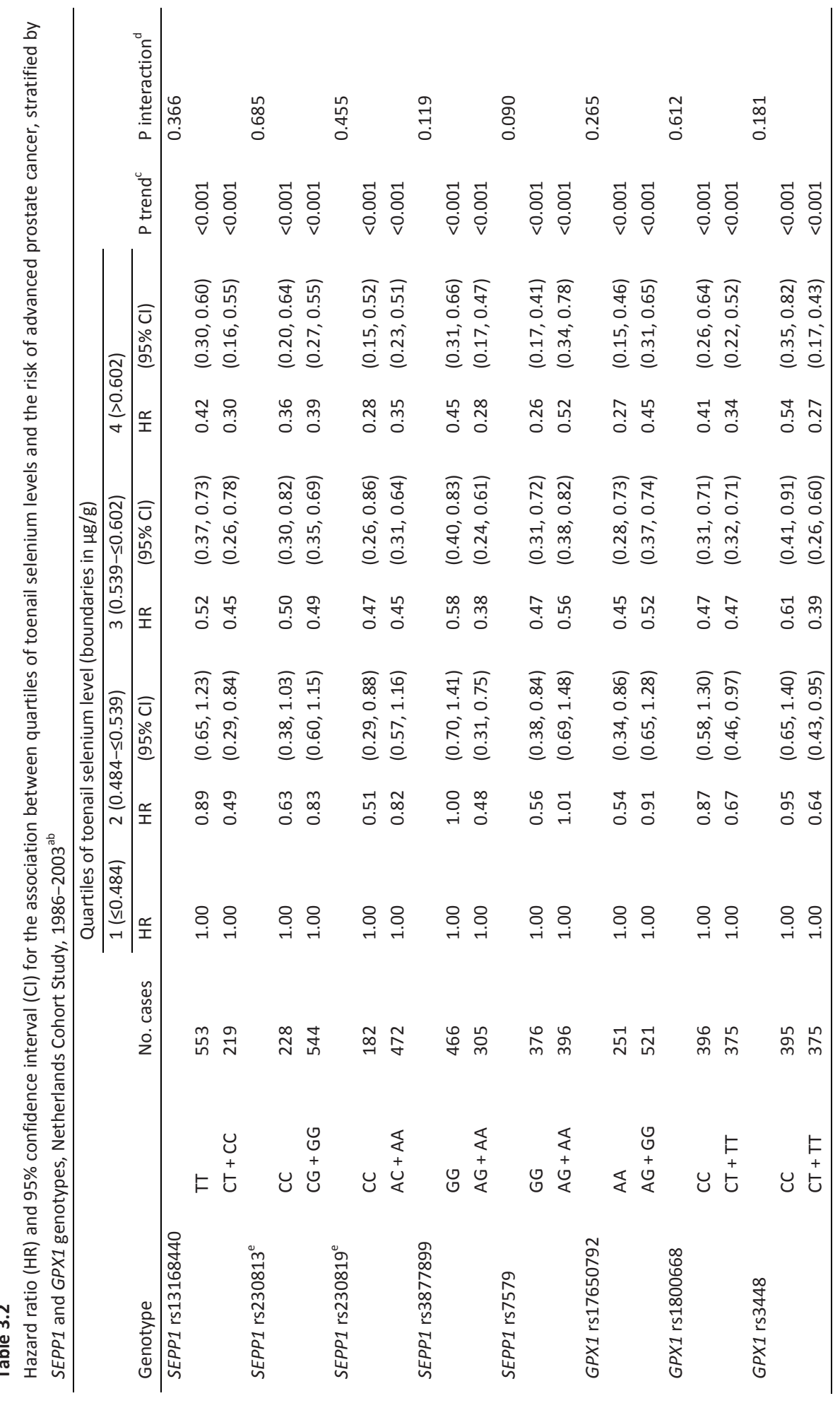




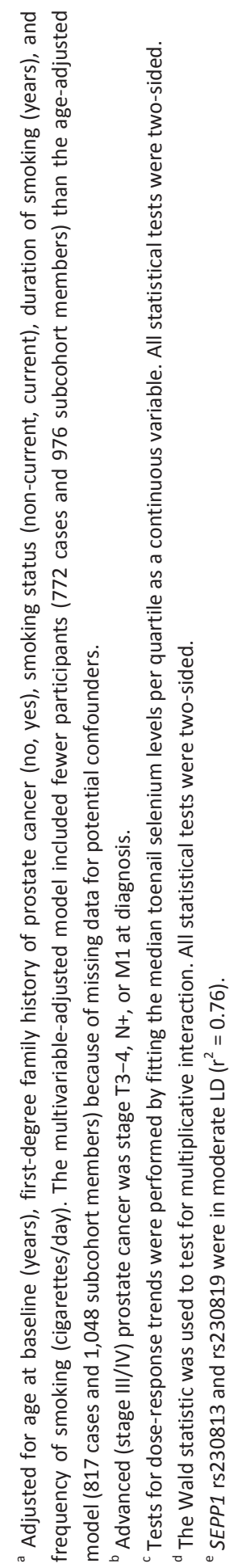




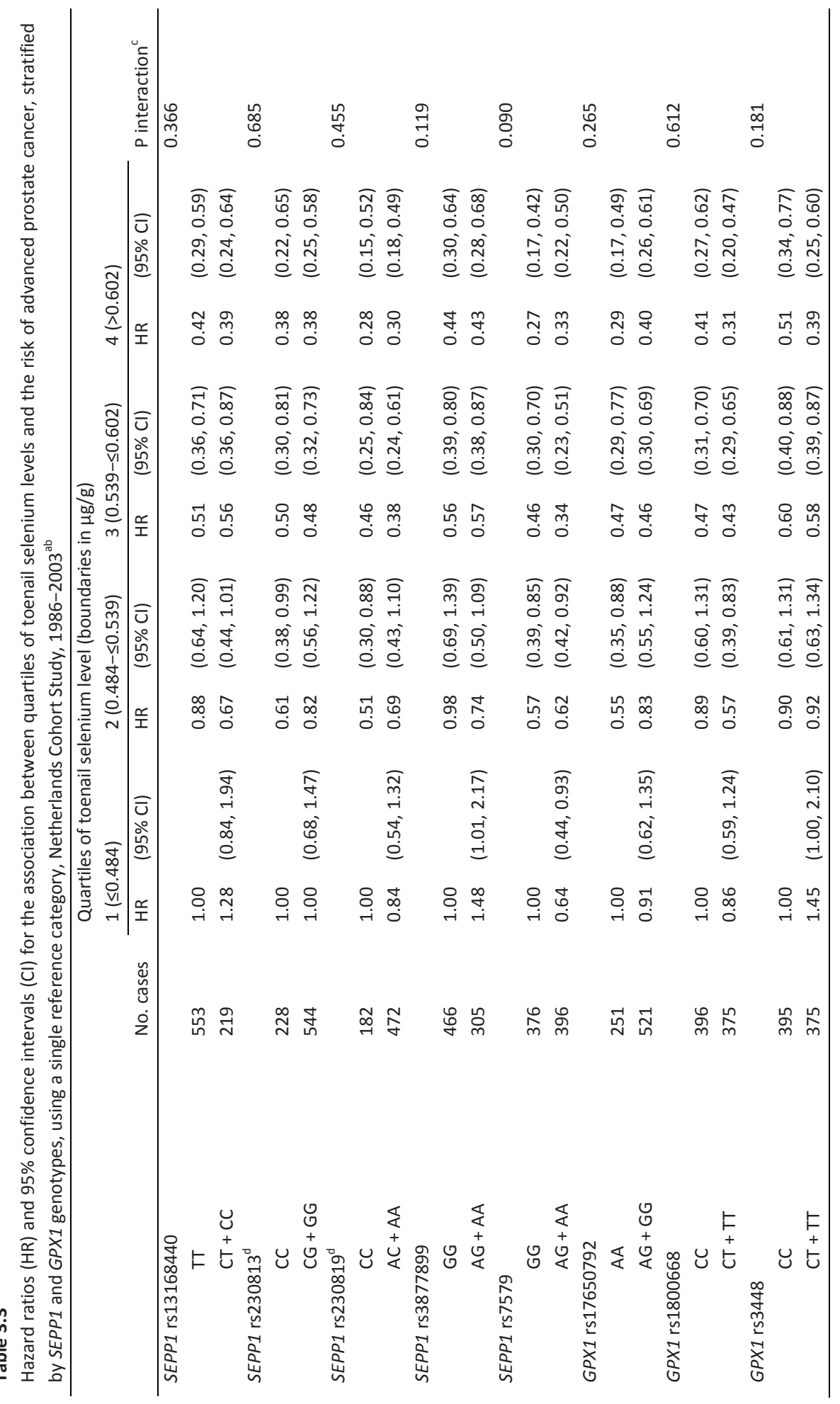




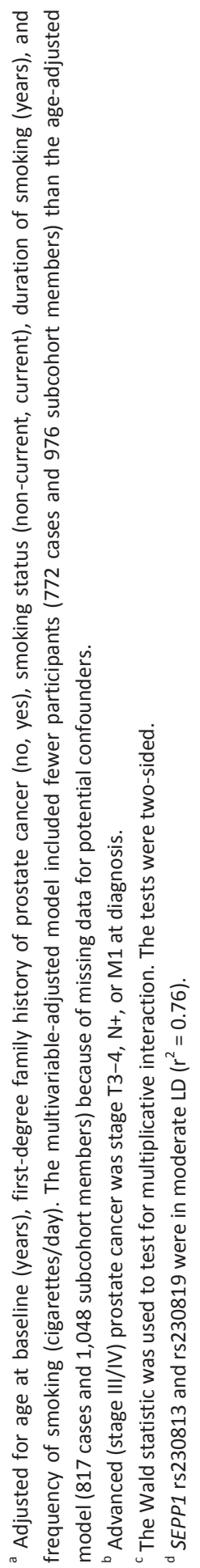




\section{Discussion}

In this long-term prospective analysis of advanced PCa risk we observed no statistical interactions between toenail selenium levels and SEPP1 and GPX1 genetic variants. Studies investigating the effect of selenium on (advanced) PCa incidence have had mixed results $[2,4,5,12]$. Our results indicate that it is unlikely that these disparate findings are the result of differences in SEPP1 and GPX1 variation. We showed that higher toenail selenium levels were associated with a decreased risk of advanced PCa in all genotype subgroups.

We observed SEPP1 and GPX1 variant main effects on advanced (stage III/IV or IV) PCa risk. These are mostly novel findings [7, 9, 22]. GPX1 rs1800668 is in high linkage disequilibrium with $r s 1050450\left(r^{2}=0.9\right)$, which resides in the coding region, results in an amino acid substitution of proline with leucine [23, 24], and may, therefore, be the true functional polymorphism.

Strengths of our study include the prospective design and large number of advanced, clinically relevant, PCa cases. We comprehensively investigated all common variation in SEPP1 and GPX1 using a tagging SNP approach. A limitation of our study is that we measured toenail selenium status once at baseline but had no repeated measurements during follow-up.

In conclusion, our data showed that low-selenium is associated with an increased risk of advanced PCa, independently of common genetic variation in SEPP1 and GPX1. Specific SEPP1 and GPX1 variants may, however, have a main effect on advanced PCa risk. The null findings of prior studies of selenium and PCa may at least partially be explained by the possibility that additional selenium intake only benefits men with relatively low selenium levels and not those who are selenium replete $[6,7,12]$.

\section{References}

1. Papp LV, Lu J, Holmgren A, Khanna KK. From selenium to selenoproteins: synthesis, identity, and their role in human health. Antioxid Redox Signal 2007;9(7):775-806.

2. Hurst R, Hooper L, Norat T, Lau R, Aune D, Greenwood DC, Vieira R, Collings R, Harvey L, Sterne JA, Beynon R, Savovic J, Fairweather-Tait SJ. Selenium and prostate cancer: systematic review and metaanalysis. Am J Clin Nutr 2012;96(1):111-122.

3. Peters U, Takata Y. Selenium and the prevention of prostate and colorectal cancer. Mol Nutr Food Res 2008;52(11):1261-1272.

4. Duffield-Lillico AJ, Dalkin BL, Reid ME, Turnbull BW, Slate EH, Jacobs ET, Marshall JR, Clark LC. Selenium supplementation, baseline plasma selenium status and incidence of prostate cancer: an analysis of the complete treatment period of the Nutritional Prevention of Cancer Trial. BJU Int 2003;91(7):608-612.

5. Klein EA, Thompson IM, Jr., Tangen CM, Crowley JJ, Lucia MS, Goodman PJ, Minasian LM, Ford LG, Parnes HL, Gaziano JM, Karp DD, Lieber MM, Walther PJ, Klotz L, Parsons JK, Chin JL, Darke AK, Lippman SM, Goodman GE, Meyskens FL, Jr., Baker LH. Vitamin E and the risk of prostate cancer: the Selenium and Vitamin E Cancer Prevention Trial (SELECT). Jama 2011;306(14):1549-1556.

6. Nicastro HL, Dunn BK. Selenium and Prostate Cancer Prevention: Insights from the Selenium and Vitamin E Cancer Prevention Trial (SELECT). Nutrients 2013;5(4):1122-1148. 
7. Rayman MP. Selenium and human health. Lancet 2012;379(9822):1256-1268.

8. Meplan C, Hesketh J. Selenium and Cancer: A Story that Should not be Forgotten-Insights from Genomics. Cancer treatment and research 2014;159:145-166.

9. Penney KL, Li H, Mucci LA, Loda M, Sesso HD, Stampfer MJ, Ma J. Selenoprotein P genetic variants and mrna expression, circulating selenium, and prostate cancer risk and survival. Prostate 2013;73(7):700705.

10. Burk RF, Hill KE. Selenoprotein P-expression, functions, and roles in mammals. Biochim Biophys Acta 2009;1790(11):1441-1447.

11. Brigelius-Flohe R, Kipp A. Glutathione peroxidases in different stages of carcinogenesis. Biochim Biophys Acta 2009;1790(11):1555-1568.

12. Geybels MS, Verhage BA, van Schooten FJ, Goldbohm RA, van den Brandt PA. Advanced prostate cancer risk in relation to toenail selenium levels. J Natl Cancer Inst 2013;105(18):1394-1401.

13. van den Brandt PA, Goldbohm RA, van 't Veer P, Volovics A, Hermus RJ, Sturmans F. A large-scale prospective cohort study on diet and cancer in The Netherlands. J Clin Epidemiol 1990;43(3):285-295.

14. van den Brandt PA, Schouten LJ, Goldbohm RA, Dorant E, Hunen PM. Development of a record linkage protocol for use in the Dutch Cancer Registry for epidemiological research. Int J Epidemiol 1990;19(3):553-558.

15. Sobin L, Gospodarowicz M, Wittekind C, eds. TNM Classification of Malignant Tumors. 7th ed. Oxford, UK: Wiley-Blackwell; 2009.

16. Carlson CS, Eberle MA, Rieder MJ, Yi Q, Kruglyak L, Nickerson DA. Selecting a maximally informative set of single-nucleotide polymorphisms for association analyses using linkage disequilibrium. Am J Hum Genet 2004;74(1):106-120.

17. van Breda SG, Hogervorst JG, Schouten L, Knaapen AM, van Delft JH, Goldbohm RA, van Schooten FJ, van den Brandt PA. Toenails: an easily accessible and long-term stable source of DNA for genetic analyses in large-scale epidemiological studies. Clin Chem 2007;53(6):1168-1170.

18. van den Brandt PA, Goldbohm RA, van't Veer P, Bode P, Hermus RJ, Sturmans F. Predictors of toenail selenium levels in men and women. Cancer Epidemiol Biomarkers Prev 1993;2(2):107-112.

19. Grambsch PM, Therneau TM. Proportional hazards tests and diagnostics based on weighted residuals. Biometrika 1994;81(3):515-526.

20. Barlow WE, Ichikawa L, Rosner D, Izumi S. Analysis of case-cohort designs. J Clin Epidemiol 1999;52(12):1165-1172.

21. Lin DY, Wei LY. The robust inference for the Cox proportional hazards model. J Am Stat Assoc. 1989;84:1074-1078.

22. Geybels MS, Hutter CM, Kwon EM, Ostrander EA, Fu R, Feng Z, Stanford JL, Peters U. Variation in selenoenzyme genes and prostate cancer risk and survival. Prostate 2013;73(7):734-742.

23. Bhatti P, Stewart PA, Hutchinson A, Rothman N, Linet MS, Inskip PD, Rajaraman P. Lead exposure, polymorphisms in genes related to oxidative stress, and risk of adult brain tumors. Cancer Epidemiol Biomarkers Prev 2009;18(6):1841-1848.

24. Foster CB, Aswath K, Chanock SJ, McKay HF, Peters U. Polymorphism analysis of six selenoprotein genes: support for a selective sweep at the glutathione peroxidase 1 locus (3p21) in Asian populations. BMC Genet 2006;7:56. 


\section{Appendix 1: Haplotype analysis}

Linkage disequilibrium (LD) plots were constructed using Haploview to explore the LD structure of the data [1]. Haplotype blocks were assembled in Haploview using the method described by Gabriel et al. [2], and haplotype risk within each block was assessed.

Figure A1.1 shows the LD plot for SEPP1. One haplotype block was identified and that block consisted of SEPP1 rs7579, which had an independent association with stage IV PCa risk (Table 3.1). There were five haplotypes in this block; four, which carried the G risk allele of SEPP1 rs7579. We found no evidence of an association with advanced PCa risk for any of the haplotypes (Table A1.1).

Figure A1.2 shows the LD plot for GPX1. One haplotype block was identified and that block included all three SNPs. GPX1 rs17650792 and rs1800668 had an independent association with advanced PCa risk (Table 3.1). There were three haplotypes in this block; one, which carried the G risk allele of rs17650792 and the C risk allele of rs1800668. This haplotype was not associated with advanced PCa risk (Table A1.2).

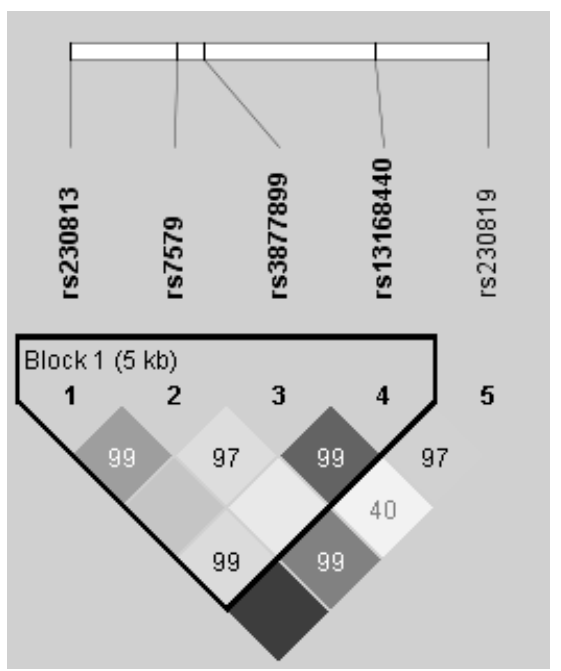

\section{Figure A1.1}

Linkage disequilibrium across SEPP1 SNPs in subcohort members, Netherlands Cohort Study Darker shading indicates higher degrees of pairwise $r^{2}$ correlation, such that $r^{2}=1$ is shown as black and $r^{2}=$ 0 is shown as white. SNPs rs230813 and rs230819 were in moderate LD $\left(r^{2}=0.76\right)$. The numbers in squares represent pairwise $D^{\prime}$ (first two decimal numbers shown), with blank boxes indicating $D^{\prime}=1$. 


\section{Table A1.1}

Hazard ratio (HR) and $95 \%$ confidence interval $(\mathrm{Cl})$ for the association of SEPP1 haplotypes and risk of advanced prostate cancer, Netherlands Cohort Study, 1986-2003 ${ }^{\text {abc }}$

\begin{tabular}{|c|c|c|c|c|}
\hline & \multicolumn{2}{|c|}{$\begin{array}{l}\text { Advanced (stage III/IV) } \\
\text { prostate cancer }^{d}\end{array}$} & \multicolumn{2}{|c|}{ Stage IV prostate cancer ${ }^{e}$} \\
\hline & No. cases & $\mathrm{HR}(95 \% \mathrm{Cl})$ & No. cases & $\mathrm{HR}(95 \% \mathrm{Cl})$ \\
\hline \multicolumn{5}{|c|}{ Two copies of 'GGGT' SEPP1 haplotype } \\
\hline No & 759 & 1.00 & 462 & 1.00 \\
\hline Yes & 193 & $1.00(0.81,1.22)$ & 133 & $1.13(0.89,1.42)$ \\
\hline \multicolumn{5}{|c|}{ Two copies of 'CGAC' SEPP1 haplotype } \\
\hline No & 926 & 1.00 & 578 & 1.00 \\
\hline Yes & 26 & $1.30(0.78,2.16)$ & 17 & $1.36(0.76,2.45)$ \\
\hline \multicolumn{5}{|c|}{ Two copies of 'CGAT' SEPP1 haplotype } \\
\hline No & 946 & 1.00 & 591 & 1.00 \\
\hline Yes & 6 & $0.95(0.35,2.58)$ & 4 & $1.02(0.32,3.23)$ \\
\hline
\end{tabular}

${ }^{a}$ All models were adjusted for age at baseline (years).

${ }^{b}$ One of the haplotypes (CGGT) was not investigated because only 1 case carried two copies of the haplotype.

c The G risk allele of rs7579, which was marginally associated with advanced prostate cancer risk in our study, is highlighted in bold.

${ }^{d}$ Advanced (stage III/IV) prostate cancers were stage T3-4, N+, or M1 at diagnosis.

e Stage IV prostate cancers were stage $\mathrm{T} 4, \mathrm{~N}+$, or $\mathrm{M} 1$ at diagnosis.

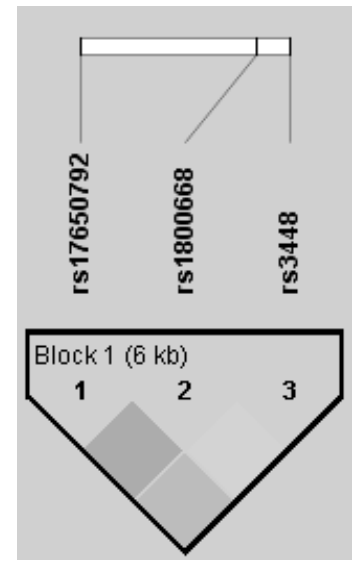

Figure A1.2

Linkage disequilibrium across GPX1 SNPs in subcohort members, Netherlands Cohort Study Darker shading indicates higher degrees of pairwise $r^{2}$ correlation, such that $r^{2}=1$ is shown as black and $r^{2}=$ 0 is shown as white. All pairwise $D^{\prime}$ were 1. 


\section{Table A1.2}

Hazard ratio $(\mathrm{HR})$ and $95 \%$ confidence interval $(\mathrm{Cl})$ for the association of GPX1 haplotypes and risk of advanced prostate cancer, Netherlands Cohort Study, 1986-2003 ${ }^{\text {ab }}$

\begin{tabular}{cllll}
\hline & \multicolumn{2}{l}{$\begin{array}{l}\text { Advanced (stage III/IV) } \\
\text { prostate cancer }\end{array}$} & \multicolumn{2}{l}{ Stage IV prostate cancer } \\
& No. cases & HR $(95 \% \mathrm{Cl})$ & No. cases & HR $(95 \% \mathrm{Cl})$ \\
\hline Two copies of 'GGC' GPX1 haplotype & & & & \\
No & 770 & 1.00 & 483 & 1.00 \\
Yes & 182 & $1.20(0.97,1.48)$ & 112 & $1.18(0.92,1.50)$ \\
\hline
\end{tabular}

${ }^{\text {a }}$ All models were adjusted for age at baseline (years).

${ }^{\mathrm{b}}$ The $\mathrm{G}$ risk allele of rs17650792 and the G risk allele of rs1800688, which were both marginally associated with advanced prostate cancer risk in our study, are highlighted in bold.

${ }^{c}$ Advanced (stage III/IV) prostate cancers were stage $\mathrm{T3}-4, \mathrm{~N}+$, or $\mathrm{M} 1$ at diagnosis.

${ }^{\mathrm{d}}$ Stage IV prostate cancers were stage $\mathrm{T} 4, \mathrm{~N}+$, or $\mathrm{M} 1$ at diagnosis.

\section{References}

1. Barrett JC, Fry B, Maller J, et al. Haploview: analysis and visualization of LD and haplotype maps. Bioinformatics 2005;21(2):263-265.

2. Gabriel SB, Schaffner SF, Nguyen $\mathrm{H}$, et al. The structure of haplotype blocks in the human genome. Science 2002;296(5576):2225-2229. 

Chapter 4: Measures of Combined Antioxidant and

Pro-oxidant Exposures and Risk of Overall and

\section{Advanced Stage Prostate Cancer}

Milan S. Geybels

Bas A.J. Verhage

Frederik J. van Schooten

Piet A. van den Brandt

Annals of Epidemiology 2012;22(11):814-820. 


\begin{abstract}
Background

Oxidative stress is possibly related to prostate carcinogenesis. We constructed a dietary antioxidant score, which is a measure of combined antioxidant intake, and an oxidative balance score, which is a measure of combined pro- and antioxidant intake. We hypothesized that both scores are inversely associated with prostate cancer (PCa) risk.
\end{abstract}

\title{
Methods
}

We conducted a case-cohort study among 58,279 men in the Netherlands Cohort Study. Cohort members completed a baseline questionnaire. From 1986 to 2003, 3,451 PCa patients were identified including 1,196 advanced cancers (stage III or IV). The antioxidant score and the oxidative balance score were created by summing quartile and category scores of individual score constituents, which had an equal weight. Prooxidants were scored in the opposite way to antioxidants.

\section{Results}

Both the antioxidant and oxidative balance score were not associated with risk of overall PCa or PCa subgroups based on disease stage. Most score constituents were not associated with PCa risk. Total catechin intake was associated with a decreased risk of stage IV PCa (HR for highest versus lowest quartile $=0.76 ; 95 \% \mathrm{Cl}: 0.59,0.98$ ).

\section{Conclusion}

We conclude that the antioxidant score and oxidative balance score were not associated with PCa risk. 


\section{Introduction}

Prostate cancer ( $\mathrm{PCa}$ ) is the most frequent cancer among males in developed countries [1]. Compared to other common cancers such as breast and lung cancer, little is known about risk factors for PCa [2-4].

A possibly important pathway in the age-related development and progression of PCa is the oxidative stress pathway [5-9]. Oxidative stress is an imbalance between the generation of reactive oxygen species (ROS) and antioxidant protection in favor of the former [10]. Intracellular levels of ROS are maintained by a complex network of antioxidant enzymes and metabolites [10, 11]. Many antioxidant metabolites are obtained from dietary sources. Examples of dietary antioxidants are vitamin C, vitamin $E$, selenium, and several carotenoids and flavonoids [11]. Human beings are also exposed to a whole range of dietary and non-dietary components that can directly or indirectly induce the production of ROS. Examples of such pro-oxidants are dietary iron, cigarette smoke, and alcohol [12-14].

Observational studies of PCa indicated that selenium and lycopene have a probable preventive effect on $\mathrm{PCa}$, but evidence for other antioxidants and prooxidants is limited [4]. A number of human clinical trials were conducted to test the role of dietary antioxidants in the prevention of PCa and collective data from these trials indicate that antioxidant supplementation does not prevent PCa [6].

A number of observational studies described different antioxidant scores [15-18]. These scores were constructed by summing quantile scores of antioxidant nutrient intake or level, where individual nutrients were given an equal weight. A high score reflected a high overall antioxidant status. One nested case-control study investigated the association between antioxidant score (based on plasma levels) and PCa risk and showed that the score was inversely associated with risk of overall PCa and that this association was more pronounced for advanced PCa [16].

Some other studies used an oxidative balance score [19-23]. An oxidative balance score is constructed by summing quantile or category scores of both antioxidant and prooxidant intake or level where pro- and antioxidants are scored in the opposite way. One small population-based case-control study showed an inverse association between the oxidative balance score and PCa risk [20], but this was not confirmed by a more recent prospective cohort study [19].

The goal of our study was to further investigate the associations between antioxidant score, oxidative balance score, individual score constituents, and risk of PCa. This investigation was carried out within the framework of The Netherlands Cohort Study, a large prospective cohort study that included 58,279 men. We have data on numerous pro- and antioxidants. Since PCa risk factors may differ for 
subgroups based on the pathologic manifestations of this disease [24], we additionally investigated risk of PCa subgroups based on disease stage.

\section{Methods}

\section{Study population}

The Netherlands Cohort Study (NLCS) is an ongoing prospective cohort study that started in September 1986. The NLCS includes 58,279 men, aged 55 to 69 years at baseline. All cohort members completed a baseline questionnaire [25]. Follow-up for incident cancer has been established by record linkage to the Netherlands cancer registries and the pathology registry PALGA [26]. The completeness of cancer follow-up through linkage with these cancer registries was assessed to be at least $96 \%$ [27]. The NLCS uses a case-cohort approach for data processing and analysis; case subjects were derived from the entire cohort and the person-time experience was estimated from a subcohort randomly sampled from the full cohort at baseline [28].

The present study is based on a follow-up of 17.3 years (September 1986 to December 2003). In our analysis, we excluded all cases with prevalent cancer other than skin cancer at baseline and individuals with incomplete or inconsistent dietary data. Our study included 3,451 incident PCa cases and 2,191 subcohort members.

The NLCS has been approved by the institutional review boards of the TNO Nutrition and Food Research Institute (Zeist, the Netherlands) and Maastricht University (Maastricht, the Netherlands).

\section{Classification of prostate cancer cases}

Because of the considerable heterogeneity in the nature of PCa, we evaluated PCa subgroups based on disease stage. Advanced prostate cancers (International Union Against cancer (UICC) [29] stage III or IV) included tumors with stage T3 or T4, N+, or M1 at diagnosis. Stage IV (UICC) prostate cancers are a subset of advanced prostate cancers and included tumors with stage $\mathrm{T} 4, \mathrm{~N}+$, or $\mathrm{M} 1$ at diagnosis. Prostate cancers that were not identified as advanced cancers were classified as non-advanced prostate cancers (UICC stage I or II; T1 or T2 and NO and MO). Our study included 1,196 advanced cancers and 2,039 non-advanced cancers. Of all advanced prostate cancers, 753 were stage IV prostate cancers.

\section{Exposure assessment}

Dietary intakes of study participants were derived from a food frequency questionnaire (FFQ). This dietary assessment was part of a larger questionnaire that also included questions regarding lifestyle and health. The FFQ was shown to be valid and reliable and is described in greater detail elsewhere [25, 30, 31]. 
Contents of $\beta$-carotene, lycopene, and catechins in foods and beverages were derived from food composition tables based on data gathered in the Netherlands [3234]. In our study, total catechin refers to (+)-catechin, (-)-epicatechin, (+)gallocatechin, (-)-epigallocatechin, (-)-epicatechin gallate, and (-)-epigallocatechin gallate.

\section{Antioxidant and oxidative balance score}

We constructed an antioxidant score and an oxidative balance score. The antioxidant score included intakes of vitamin C, $\beta$-carotene, lycopene, vitamin $E$, and total catechin. The oxidative balance score included these antioxidant intakes plus the variables heme iron intake, smoking status, and alcohol intake (all pro-oxidants). These antioxidant and prooxidant exposures were included in previously described antioxidant scores and oxidative balance scores [15-23].

All continuous score variables were divided into quartiles based on the distribution of these variables among male subcohort members. Men in the first, second, third, and fourth quartile of antioxidant intake were assigned zero, one, two, and three points respectively. Scoring for prooxidants was conducted in the opposite fashion. Smoking status was categorized as never (three points), former (1.5 points), or current (zero points). Alcohol consumption was categorized as abstainer (three points), 0.1 to $4 \mathrm{~g} /$ day ( 2.25 points), 5 to $14 \mathrm{~g} /$ day, ( 1.5 points), 15 to $29 \mathrm{~g} /$ day ( 0.75 points), or $\geq 30 \mathrm{~g} /$ day (zero points). The range of antioxidant score values and oxidative balance score values was 0-15 and 0-24, respectively.

\section{Data analysis}

We used Cox proportional hazards regression to obtain hazard ratios (HRs) and corresponding 95\% confidence intervals (Cls). Cut-off points for each quartile were based on the distribution of each variable in the subcohort. Tests for linear trend were carried out by taking the median values of each quartile or category and modeling exposure as a continuous variable. All models were adjusted for age at baseline as a continuous variable. The following factors, which are hypothesized or known to be associated with the studied exposures or PCa risk, were assessed as potentially confounding variables: family history of PCa among first degree relatives (yes/no), duration of smoking (years), frequency of smoking (cigarettes/day), non-occupational physical activity $(\leq 30,>30-60,>60-90,>90$ minutes/day), $\mathrm{BMI}(<23,23-<25,25-<27$, $27-<30, \geq 30$ ), height $(\mathrm{cm})$, diabetes (type I or II, yes/no), education level (primary school, lower vocational, high school, higher vocational/university), and intakes of energy (kcal/day) and calcium (g/day). We evaluated, using a backwards stepwise procedure, whether these variables changed the point estimates by at least $10 \%$. Because none did so, our final model was adjusted for age at baseline only. The 
proportional hazards assumption was tested using the scaled Schoenfeld residuals [35]. Standard errors were estimated by using the robust Huber-White sandwich estimator, to account for additional variance introduced by sampling from the cohort [36].

Overall antioxidant status might depend on exposure to toxic constituents present in cigarette smoke. Cigarette smoke is a hugely complex mixture of toxic agents that can directly or indirectly induce oxidative stress [13]. We therefore investigated if the antioxidant score was differently associated with PCa risk in subgroups based on smoking status (never, former, current). These models were additionally adjusted for duration of smoking (smoking years) and frequency of smoking (cigarettes/day). We tested for interactions by using cross-product terms in the regression model.

All P-values were two-sided, with a P-value less than 0.05 considered to be statistically significant. Analyses were performed using STATA software (release 11, STATA Corporation, College Station, TX).

\section{Results}

The baseline characteristics of our study population are shown in Table 4.1. Compared with subcohort members, PCa cases were more likely to have a first degree relative affected with PCa and less likely to have a history of diabetes. Baseline characteristics of all other factors were comparable between cases and subcohort members.

We present age-adjusted $\mathrm{HRs}$ and $95 \% \mathrm{Cls}$. The antioxidant score was not associated with overall, non-advanced (stage I or II), advanced (stage II or IV), or stage IV PCa risk (Table 4.2). Total catechin intake was associated with a decreased risk of stage IV PCa (HR for the highest versus lowest quartile $=0.76 ; 95 \% \mathrm{Cl}: 0.59,0.98$; P for trend $=0.019$ ). No significant associations were observed for overall, non-advanced, or advanced PCa risk. Associations were not observed for the other single antioxidants.

We investigated the association between the antioxidant score and PCa risk among subgroups based on smoking status. Among former smokers, the antioxidant score was associated with an increased risk of overall PCa (HR for the highest versus lowest quartile $=1.38 ; 95 \% \mathrm{Cl}$ : 1.09, 1.75; $\mathrm{P}$ for trend $=0.008$ ) (Table 4.3). No significant associations were observed among never and current smokers. The antioxidant score was not associated with non-advanced or advanced PCa risk among men with a different smoking status.

The oxidative balance score was not associated with a decreased risk of overall, non-advanced, advanced, or stage IV PCa risk (Table 4.4). The oxidative balance score was associated with a modest increased risk of non-advanced PCa (HR for highest 
versus lowest quartile $=1.20 ; 95 \% \mathrm{Cl}: 1.00,1.44 ; \mathrm{P}$ for trend $=0.031$ ). Smoking status and alcohol and heme iron intake were not associated with PCa risk. 


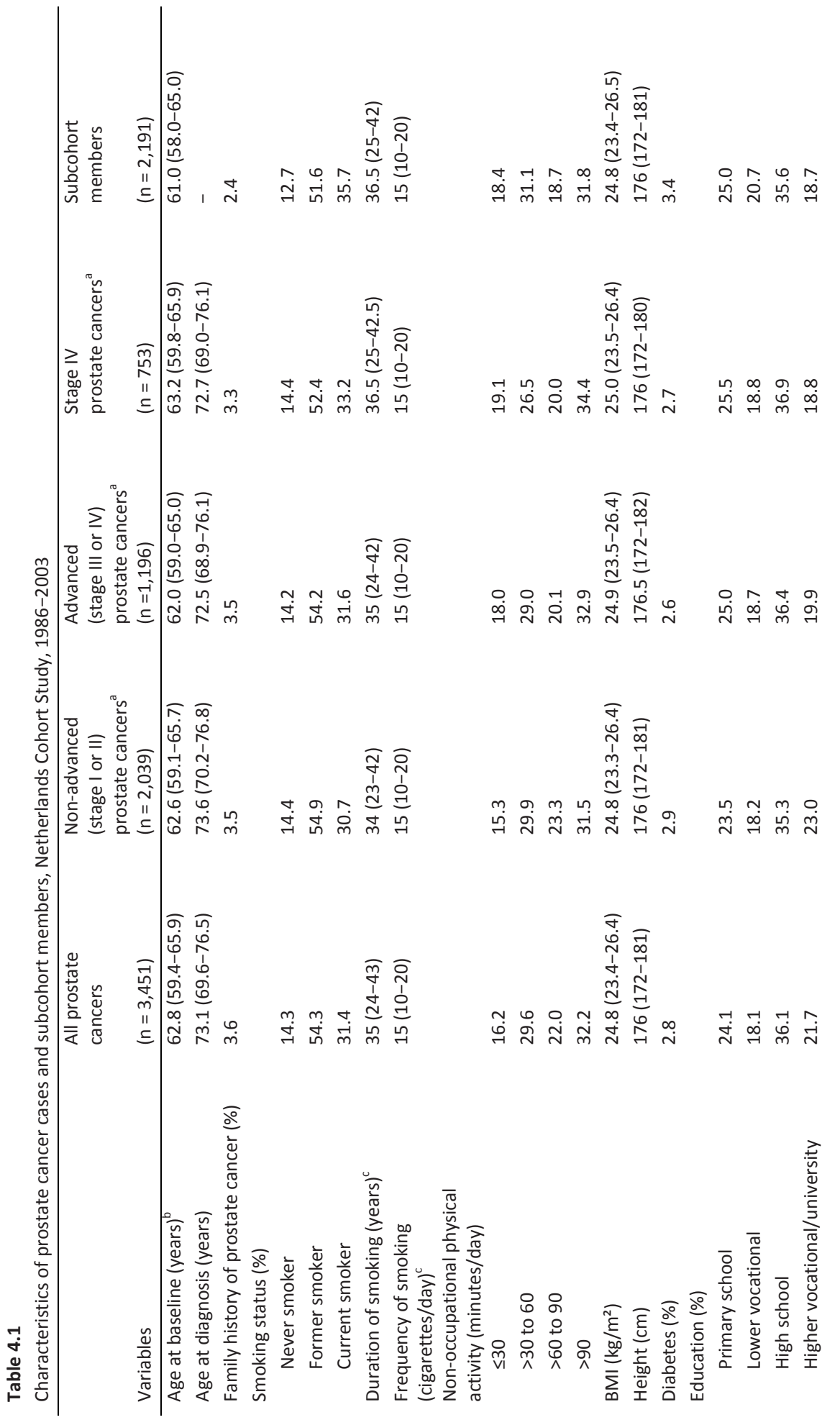




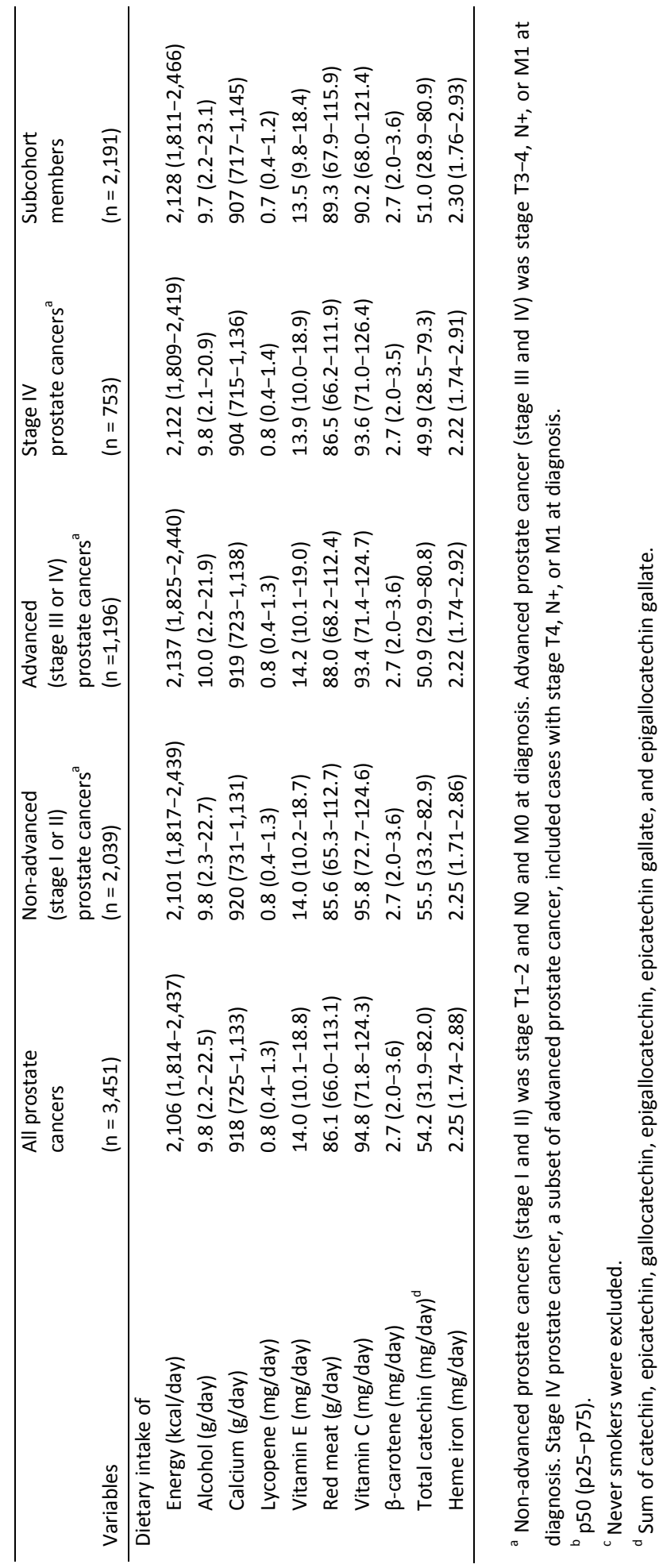




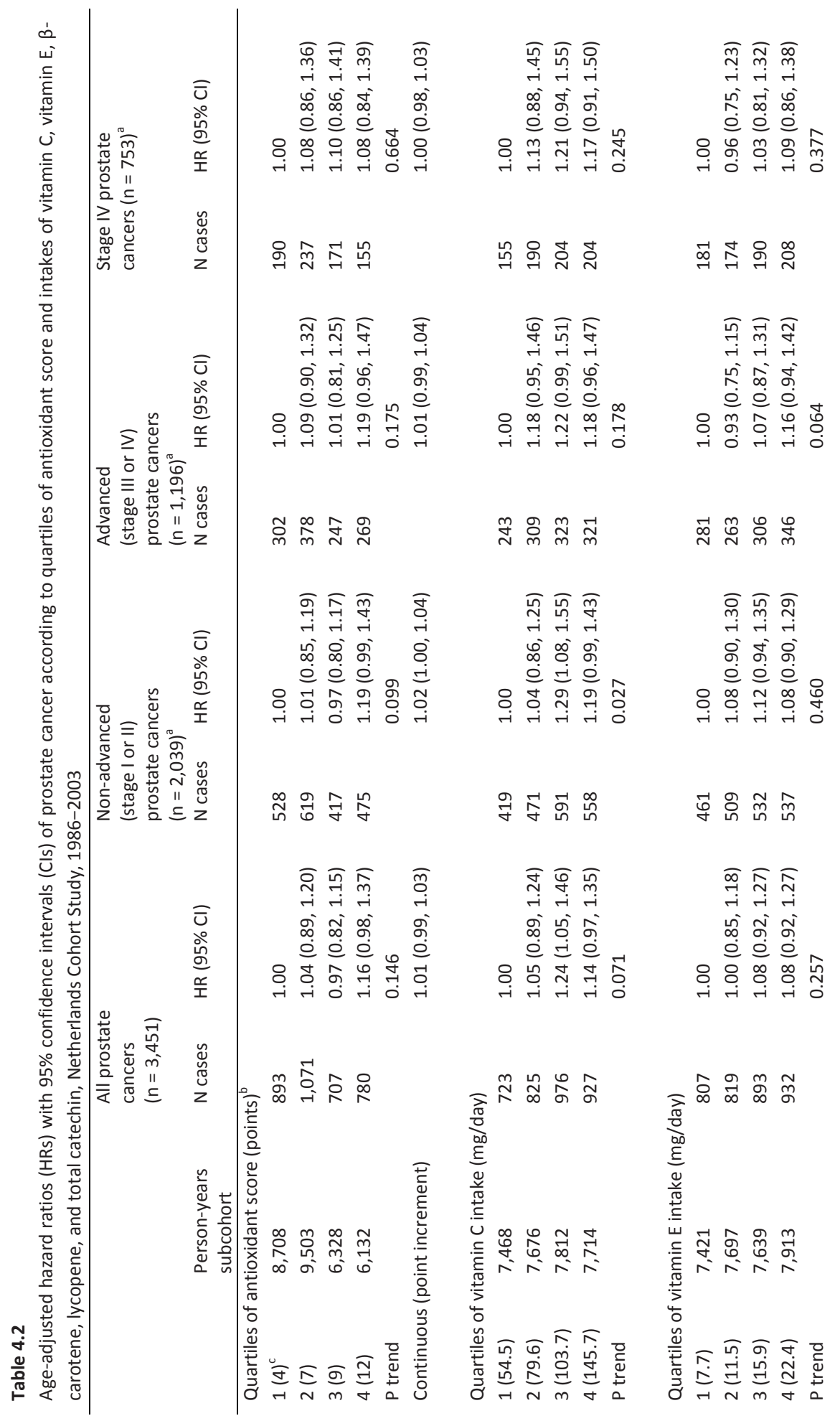




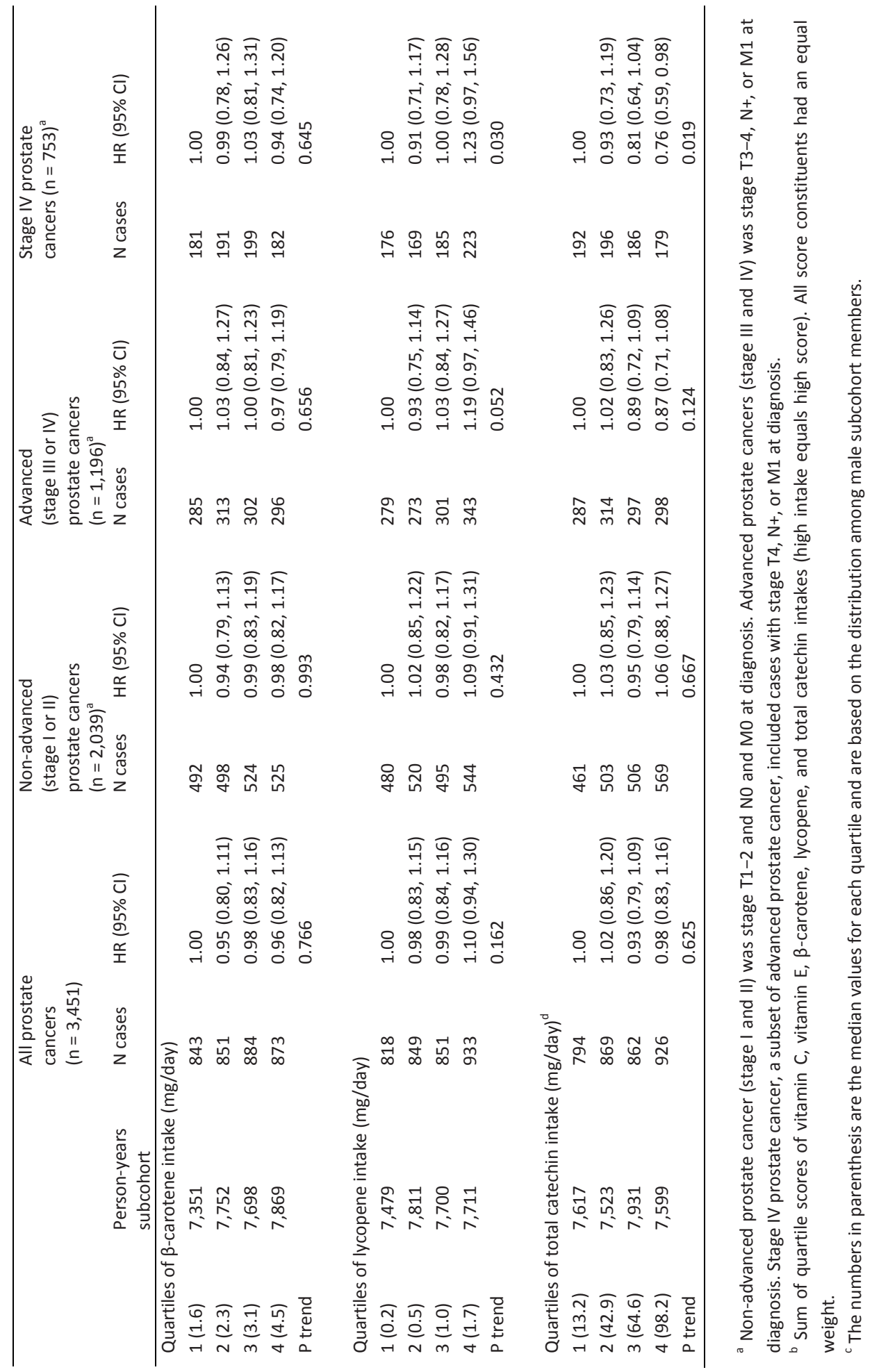




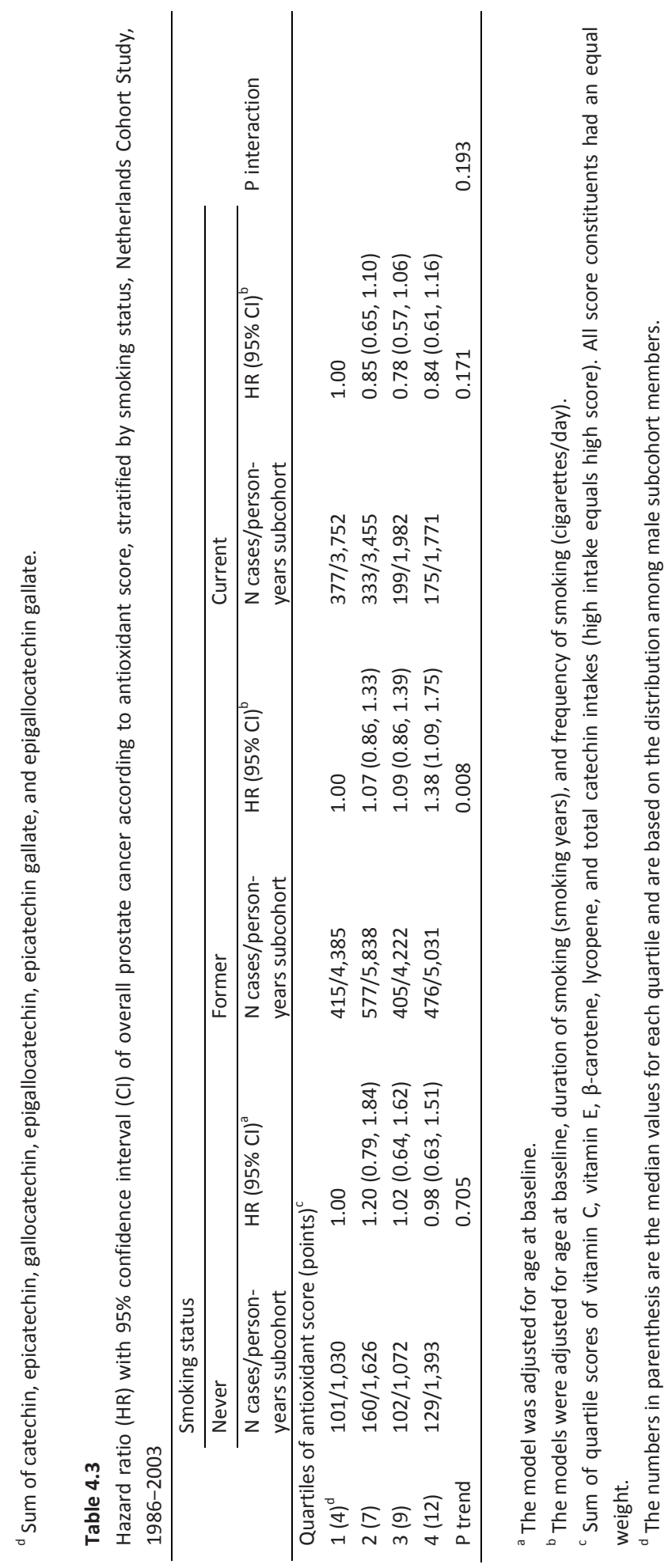




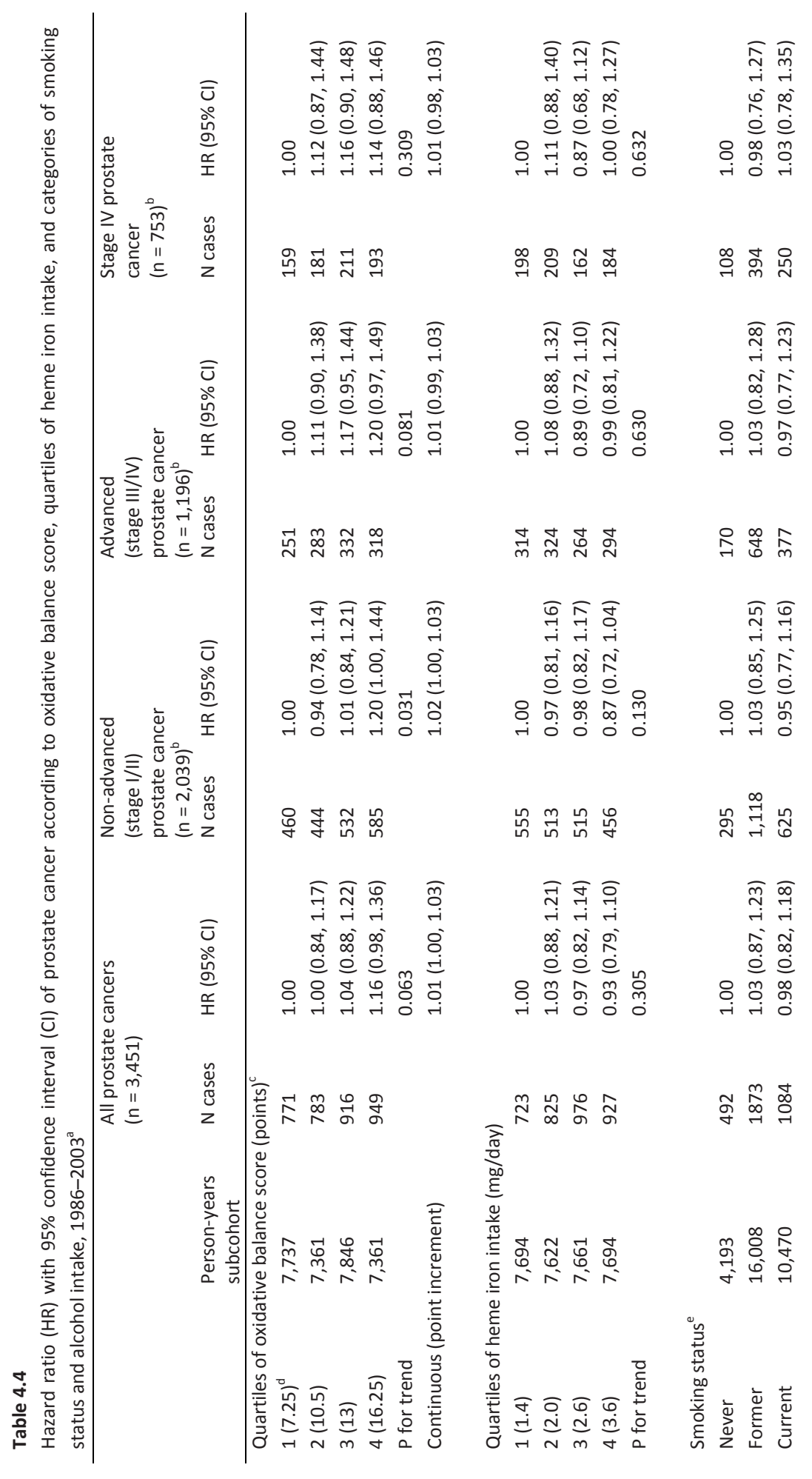




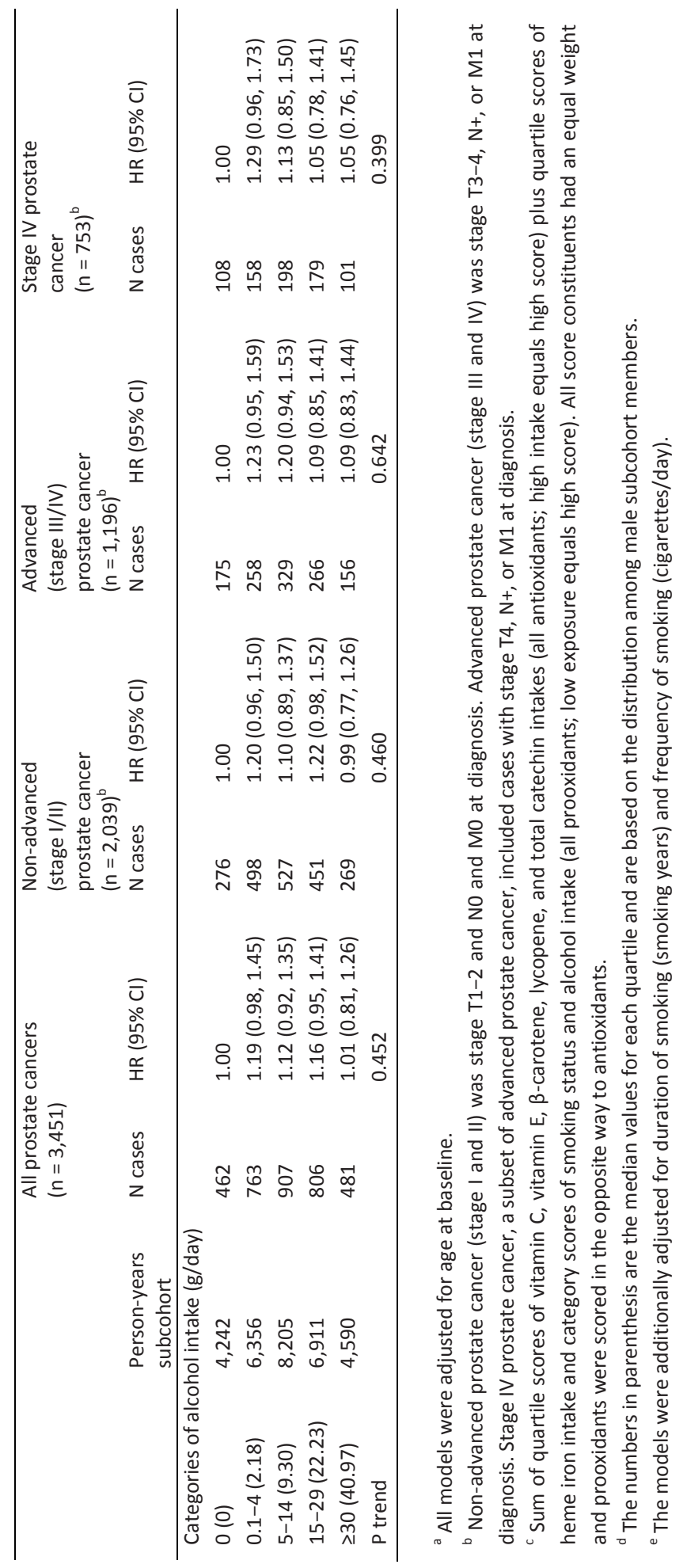




\section{Discussion}

We constructed an antioxidant score and an oxidative balance score and investigated associations between these scores and PCa risk. We found that both scores were not associated with risk of PCa or PCa subgroups based on disease stage.

All constituents of our antioxidant score were used in previously described comparable scores [15-18]. Flavonoids such as catechins and flavonols are polyphenolic antioxidants [37]. Flavonoids were included in the antioxidant score described by Wright et al. [18]. We included total catechin intake in our score because this is the major flavonoid subclass in our population. Other flavonoids were not included because (intake of) these were highly correlated with total catechin intake. Our antioxidant score incorporates antioxidant nutrients that are derived from a variety of food sources. The best sources of vitamin $\mathrm{E}$ are vegetable oils and fats. Major sources of vitamin C, $\beta$-carotene, and lycopene are fruits and vegetables. The major source of catechins is black tea.

One previous observational study investigated the association between antioxidant score and PCa risk [16]. This nested case-control study by Li et al. (496 cases and 402 controls) showed an inverse association between the antioxidant score (highest versus lowest category) and risk of overall PCa ( $\mathrm{RR}=0.60 ; 95 \% \mathrm{Cl}: 0.39,0.88$ ) and aggressive PCa (RR $=0.40 ; 95 \% \mathrm{Cl}: 0.25,0.69)$ but not non-aggressive PCa [16]. This antioxidant score was derived by summing quartile scores of selenium, $\alpha$-tocopherol, and lycopene levels in blood. This score is in many ways different from our antioxidant score. One major difference is related to the selection of score constituents. Our score included more constituents than the score by Li et al. [16] but did not include selenium status. Selenium status was not included in our score because these data were not available for 17.3 years of follow-up. Our Netherlands Cohort Study previously showed, for 6.3 years of follow-up, that toenail selenium status was associated with a reduced risk of overall PCa (HR for highest versus lowest quintile $=0.69 ; 95 \% \mathrm{Cl}: 0.48,0.99$ ) [38]. Another major difference is that while we used data on nutrient intakes Li et al. used data on antioxidant blood levels [16]. Biomarker data may provide more biologic insight since biomarkers are integral measures of intake, absorption, and metabolism of nutrients [22].

Most of our individual antioxidant score constituents were not associated with a decreased risk of PCa. The only exception is total catechin intake, which was associated with a decreased risk of stage IV PCa. Few epidemiological studies investigated associations between catechin intake and PCa risk and the few that have reported null results $[39,40]$. Our finding therefore requires confirmation. We unexpectedly observed that men in the highest compared to the lowest quartile of lycopene intake had a modest increased risk of stage IV PCa. Numerous other observational studies 
showed an inverse association between lycopene intake and PCa risk [4]. Our result may therefore be due to chance.

We investigated whether the association between the antioxidant score and PCa risk was different among men with a different smoking status. We hypothesized that male smokers compared to never and former smokers benefit more from a high overall antioxidant intake. Unexpectedly we observed that among former smokers, men in the highest compared to the lowest quartile of antioxidant score had an increased risk of overall PCa. No associations were observed in the group of never and current smokers. This finding warrants further study.

Our oxidative balance score included the same variables as our antioxidant score plus the variables heme iron intake, smoking status, and alcohol intake. Smoking status, alcohol intake, and iron intake were included in previously described oxidative balance scores (19-23). We preferred heme iron over total iron because this is the most bio-available form of dietary iron and as such is the greatest contributor to physiologic iron stores [18, 23].

One previous prospective study and case-control study investigated the association between oxidative balance score and PCa risk [19, 20]. A small case-control study by Goodman et al. (89 cases and 197 controls) showed a significant inverse association between oxidative balance score and PCa risk [20]. Odds ratios for the middle and highest categories of oxidative balance score (predominance of antioxidant exposures) compared to the lowest category (predominance of pro-oxidant exposures) were $0.29(95 \% \mathrm{Cl} ; 0.12,0.71)$ and $0.28(95 \% \mathrm{Cl} ; 0.10,0.82)$, respectively. A prospective cohort study by Agalliu et al. (661 prostate cancers of which 173 were advanced) observed no association between oxidative balance score and risk of overall PCa or PCa subgroups based on disease stage [19]. Constituents of our score were also included in the oxidative balance scores described by Goodman et al. and Agalliu et al. [19, 20]. These scores however included some additional dietary constituents such as specific carotenoids ( $\alpha$-carotene, $\beta$-cryptoxanthin, lutein, and zeaxanthin), polyunsaturated fatty acids, cruciferous vegetables, and red meat. These additional variables were not included in our score because they were highly correlated with our other score constituents. Agalliu et al. reported a maximum correlation coefficient of 0.4 between the constituents of their score [19]. In our study, much stronger correlations were observed between our original score variables and these additional score variables. Both Agalliu et al. and Goodman et al. also combined dietary and supplemental nutrient intakes $[19,20]$. This was not the case in our study. In our study, overall supplemental intake was very low and information on exact supplemental intake was not available. Another difference with the oxidative balance score by Goodman et al. is that they included the variables non-steroidal anti-inflammatory drugs (NSAIDs) use and aspirin use [20]. The motivation for that was that these drugs might prevent the 
production of ROS by inhibition of inflammatory processes. These variables were not included in our score because very few individuals in our study population reported regularly (at least 6 months) using aspirin or NSAIDs (3\% of male subcohort members for both NSAIDs and aspirin use). Overall, our result is in line with the result of the prospective study by Agalliu et al. [19], which showed that oxidative balance score is not associated with the risk of overall PCa or PCa subgroups based on disease stage.

All factors included in our scores were given an equal weight. It is however unlikely that all studied pro- and antioxidants are equally potent. Besides that, the antioxidant activity of a compound might depend on the biological activity of other antioxidants. It is known for example that vitamin $\mathrm{C}$ reacts with vitamin $\mathrm{E}$ radicals to regenerate vitamin E [11]. Furthermore, our scores were probably incomplete. Not all possible pro- and antioxidant factors were included. Several carotenoids and other antioxidant nutrients were not included because they were correlated with other antioxidants in our score. Wright et al. countered this problem by utilizing principal components analysis [18]. This technique reduces a large number of highly correlated variables to a smaller set of components that capture as much of the variability in the data as possible. We however decided to not apply this technique because it would limit the comparability of our study with other studies [21]. Besides that, there are a number of other factors that are potential important candidates to include in an antioxidant or oxidative balance score such as exposure to environmental pollutants, work related exposures, and physical activity [41, 42].

Strengths of this study include the prospective design and large number of cases. The Netherlands Cohort Study had almost complete ascertainment of PCa cases and subcohort members and we had data on many pro- and antioxidants.

In conclusion, both the antioxidant score and oxidative balance score were not associated with risk of overall PCa or PCa subgroups based on disease stage.

\section{References}

1. Jemal A, Bray F, Center MM, Ferlay J, Ward E, Forman D. Global cancer statistics. CA Cancer J Clin 2011;61:69-90.

2. Bostwick DG, Burke HB, Djakiew D, Euling S, Ho SM, Landolph J, et al. Human prostate cancer risk factors. Cancer 2004;101:2371-490.

3. Gronberg H. Prostate cancer epidemiology. Lancet 2003;361:859-64.

4. World Cancer Research Fund/American Institute for Cancer Research. Food, nutrition, physical activity, and the prevention of cancer: a global perspective. Washington DC: AICR; 2007.

5. Halliwell B. Oxidative stress and cancer: have we moved forward? Biochem J 2007;401:1-11.

6. Khandrika L, Kumar B, Koul S, Maroni P, Koul HK. Oxidative stress in prostate cancer. Cancer lett 2009;282:125-36.

7. Minelli A, Bellezza I, Conte C, Culig Z. Oxidative stress-related aging: A role for prostate cancer? Biochim Biophys Acta 2009;1795:83-91.

8. Halliwell B. Reactive species and antioxidants. Redox biology is a fundamental theme of aerobic life. Plant Physiol 2006;141:312-22. 
9. Kumar B, Koul S, Khandrika L, Meacham RB, Koul HK. Oxidative stress is inherent in prostate cancer cells and is required for aggressive phenotype. Cancer Res 2008;68:1777-85.

10. Yossepowitch O, Pinchuk I, Gur U, Neumann A, Lichtenberg D, Baniel J. Advanced but not localized prostate cancer is associated with increased oxidative stress. J Urol 2007;178:1238-43.

11. Fang YZ, Yang S, Wu G. Free radicals, antioxidants, and nutrition. Nutrition 2002;18:872-9.

12. Albano E. Alcohol, oxidative stress and free radical damage. Proc Nutr Soc 2006;65:278-90.

13. Hecht SS. Tobacco smoke carcinogens and lung cancer. J Natl Cancer Inst 1999;91:1194-210.

14. Valko M, Rhodes CJ, Moncol J, Izakovic M, Mazur M. Free radicals, metals and antioxidants in oxidative stress-induced cancer. Chem Biol Interact 2006;160:1-40.

15. Lee KM, Kang D, Park SK, et al. Nitric oxide synthase gene polymorphisms and prostate cancer risk. Carcinogenesis 2009;30:621-5.

16. Li H, Kantoff PW, Giovannucci E, Leitzman MF, Gaziano JM, Stampfer MJ, et al. Manganese superoxide dismutase polymorphism, prediagnostic antioxidant status, and risk of clinical significant prostate cancer. Cancer Res 2005;65:2498-504.

17. Terry P, Lagergren J, Ye W, Nyren O, Wolk A. Antioxidants and cancers of the esophagus and gastric cardia. Int J Cancer 2000;87:750-4.

18. Wright ME, Mayne ST, Stolzenberg-Solomon RZ, Li Z, Pietinen P, Taylor PR, et al. Development of a comprehensive dietary antioxidant index and application to lung cancer risk in a cohort of male smokers. Am J Epidemiol 2004;160:68-76.

19. Agalliu I, Kirsh VA, Kreiger N, Soskolne CL, Rohan TE. Oxidative balance score and risk of prostate cancer: Results from a case-cohort study. Cancer Epidemiol 2010;35:353-61.

20. Goodman M, Bostick RM, Dash C, Flanders WD, Mandel JS. Hypothesis: oxidative stress score as a combined measure of pro-oxidant and antioxidant exposures. Ann Epidemiol 2007;17:394-9.

21. Goodman M, Bostick RM, Dash C, Terry P, Flanders WD, Mandel J. A summary measure of pro- and antioxidant exposures and risk of incident, sporadic, colorectal adenomas. Cancer Causes Control 2008;19:1051-64.

22. Goodman M, Bostick RM, Gross M, Thyagarajan B, Dash C, Flanders WD. Combined measure of pro- and anti-oxidant exposures in relation to prostate cancer and colorectal adenoma risk: an update. Ann Epidemiol 2010;20:955-7.

23. Van Hoydonck PG, Temme EH, Schouten EG. A dietary oxidative balance score of vitamin C, betacarotene and iron intakes and mortality risk in male smoking Belgians. J Nutr 2002;132:756-61.

24. Giovannucci E, Liu Y, Platz EA, Stampfer MJ, Willett WC. Risk factors for prostate cancer incidence and progression in the health professionals follow-up study. Int J Cancer 2007;121:1571-8.

25. van den Brandt PA, Goldbohm RA, van 't Veer P, Volovics A, Hermus RJ, Sturmans F. A large-scale prospective cohort study on diet and cancer in The Netherlands. J Clin Epidemiol 1990;43:285-95.

26. van den Brandt PA, Schouten LJ, Goldbohm RA, Dorant E, Hunen PM. Development of a record linkage protocol for use in the Dutch Cancer Registry for Epidemiological Research. Int J Epidemiol 1990;19:5538.

27. Goldbohm R, van den Brandt P, Dorant E. Estimation of the coverage of Dutch municipalities by cancer registries and PALGA based on hospital discharge data. Tijdschr Soc Gezondheidsz 1994:80-4.

28. Barlow WE, Ichikawa L, Rosner D, Izumi S. Analysis of case-cohort designs. J Clin Epidemiol 1999;52:1165-72.

29. Sobin L, Gospodarowicz M, Wittekind C, eds. TNM Classification of Malignant Tumors. 7th ed. Oxford, UK: Wiley-Blackwell; 2009.

30. Goldbohm RA, van den Brandt PA, Brants HA, van't Veer P, Al M, Sturmans F, et al. Validation of a dietary questionnaire used in a large-scale prospective cohort study on diet and cancer. Eur J Clin Nutr 1994;48:253-65.

31. Goldbohm RA, van't Veer P, van den Brandt PA, van't Hof MA, Brants HAM, Sturmans F, et al. Reproducibility of a food frequency questionnaire and stability of dietary habits determined from five annually repeated measurements. Eur J Clin Nutr 1995;49:420-9.

32. Arts IC, van De Putte B, Hollman PC. Catechin contents of foods commonly consumed in The Netherlands. 2. Tea, wine, fruit juices, and chocolate milk. J Agric Food Chem 2000;48:1752-7.

33. Arts IC, van de Putte B, Hollman PC. Catechin contents of foods commonly consumed in The Netherlands. 1. Fruits, vegetables, staple foods, and processed foods. J Agric Food Chem 2000;48:174651. 
34. Goldbohm RA, Brants HA, Hulshof KF, van den Brandt PA. The contribution of various foods to intake of vitamin A and carotenoids in The Netherlands. Int J Vitam Nutr Res 1998;68:378-83.

35. Schoenfeld D. Partial residuals for the proportional hazards regression model. Biometrika 1982;1:23941.

36. Lin DY, Wei, L.Y. The robust inference for the Cox proportional hazards model. J Am Stat Assoc 1989;84:1074-8.

37. Pietta PG. Flavonoids as antioxidants. J Nat Prod 2000;63:1035-42.

38. van den Brandt PA, Zeegers MP, Bode P, Goldbohm RA. Toenail selenium levels and the subsequent risk of prostate cancer: a prospective cohort study. Cancer Epidemiol Biomarkers Prev 2003;12:866-71.

39. Bosetti C, Bravi F, Talamini R, et al. Flavonoids and prostate cancer risk: a study in Italy. Nutr Cancer 2006;56:123-7.

40. Mursu J, Nurmi T, Tuomainen TP, Salonen JT, Pukkala E, Voutilainen S. Intake of flavonoids and risk of cancer in Finnish men: The Kuopio Ischaemic Heart Disease Risk Factor Study. Int J Cancer 2008;123:660-3.

41. Gomez-Cabrera MC, Domenech E, Vina J. Moderate exercise is an antioxidant: upregulation of antioxidant genes by training. Free Radic Biol Med 2008;44:126-31.

42. Mena S, Ortega A, Estrela JM. Oxidative stress in environmental-induced carcinogenesis. Mutat Res 2009;674:36-44. 

Chapter 5: Dietary Flavonoid Intake, Black Tea

Consumption, and Risk of Overall and Advanced Stage Prostate Cancer

Milan S. Geybels

Bas A.J. Verhage

Ilja C.W. Arts

Frederik J. van Schooten

R. Alexandra Goldbohm

Piet A. van den Brandt

American Journal of Epidemiology 2013;177(12):1388-1398. 


\section{Abstract}

\section{Background}

Flavonoids are natural antioxidants found in various foods and a major dietary source is black tea. There is some experimental evidence that flavonoids may prevent prostate cancer (PCa).

\section{Methods}

We investigated the associations between flavonoid intake, black tea consumption, and PCa risk in the Netherlands Cohort study, which included 58,279 men that provided detailed baseline information on several cancer risk factors. From 1986 to 2003, 3,362 prostate cancers were identified including 1,164 advanced (stage III and IV) cancers. Cox proportional hazards regression using the case-cohort approach was used to estimate hazard ratios (HRs) and 95\% confidence intervals (Cls).

\section{Results}

Intake of total catechin, epicatechin, kaempferol, and myricetin and consumption of black tea were associated with a decreased risk of stage III/IV or IV PCa. HRs of stage III/IV and IV PCa for the highest versus the lowest category of black tea consumption ( $\geq 5$ versus $\leq 1 \mathrm{cups} /$ day) were 0.75 (95\% Cl: $0.59,0.97)$ and 0.67 (95\% Cl: $0.50,0.91)$ respectively. No associations were observed for overall and non-advanced $\mathrm{PCa}$.

\section{Conclusion}

In conclusion, dietary flavonoid intake and black tea consumption were associated with a decreased risk of advanced PCa. 


\section{Introduction}

Flavonoids are a large group of polyphenolic antioxidants that are found in various foods of plant origin [1, 2]. These compounds are classified into different subclasses and for the major subclasses of flavonols and catechins, comprehensive data on their content in foods are available [1]. Flavonoids are abundantly present in green tea and black tea, which are both produced from the leaves of the plant Camellia sinensis [3-5]. The key chemical differences between both tea types result from post-harvest processing. In the processing of green tea, fresh tea leaves are heat-treated immediately after harvest, resulting in minimal oxidation of the naturally occurring polyphenols. In the processing of black tea, tea leaves are dried and crushed upon harvesting to encourage oxidation, which converts part of the endogenous tea polyphenols (primarily catechins) to other polymeric polyphenols such as theaflavins and thearubigens [5]. Besides tea other foods contribute significantly to flavonoid intake and these include apples, onions, chocolate, legumes, cabbages, and leafy vegetables [3, 4].

Several lines of experimental evidence suggest that flavonoids have specific chemopreventive effects on PCa. Epigallocatechin-3-gallate (EGCG) is the most abundant green tea catechin and the most studied flavonoid and it has significant growth inhibitory properties in PCa cells [6]. A number of animal studies showed that both green tea extracts and black tea extracts have inhibitory effects on PCa growth [68]. Most of these studies used polyphenolic fractions isolated from green tea or purified EGCG. The anticancer effects of flavonoids may be related to their antioxidant effects or other properties such as effects on enzyme activities, anti-inflammatory effects, and inhibition of angiogenesis [9-11].

There has been one small proof-of-principle clinical trial among 60 men with highgrade prostate intra-epithelial neoplasia that investigated the chemopreventive effects of green tea catechins (GTCs) on PCa $[12,13]$. High-grade prostate intra-epithelial neoplasia is a likely precursor of PCa [14]. In this double-blind, placebo-controlled study, participants received three (200 mg) GTCs capsules per day. After 1 year, only one tumor was diagnosed among the 30 GTCs-treated men, whereas nine cancers were found among the 30 placebo-treated men.

Unlike the experimental evidence, the few observational studies of flavonoids and PCa conducted to date mostly reported null results [15-20]. Observational studies of tea and PCa were generally inconclusive $[5,21,22]$. It is important to note that most of these studies were limited by a small number of cases and that studies of tea consumption and PCa risk were often limited by a narrow range of tea intake. An additional consideration is that while risk factors for PCa are likely to differ for subgroups based on disease stage or aggressiveness [23], few of these prior 
investigations considered the clinical or pathologic manifestations of PCa in the study design and interpretation.

We therefore investigated the associations between dietary flavonoid intake, black tea consumption, and PCa risk in a large cohort from the Netherlands with a relatively wide range of these exposures. We evaluated intake of total catechin, total flavonol, and specific catechins (catechin and epicatechin) and flavonols (quercetin, kaempferol, and myricetin). We investigated risk of both overall PCa and PCa subgroups based on disease stage since associations may be different in these subgroups.

\section{Methods}

\section{Study population}

The Netherlands Cohort Study (NLCS) is an ongoing prospective cohort study that started in September 1986. The NLCS includes 58,279 men, aged 55 to 69 years at baseline. All cohort members completed a baseline questionnaire [24]. Follow-up for incident cancer has been established by record linkage to the Netherlands cancer registries and the pathology registry PALGA [25]. The completeness of cancer follow-up through linkage with these cancer registries was assessed to be at least $96 \%$ [26]. The NLCS uses a case-cohort approach for data processing and analysis; case subjects were derived from the entire cohort and the person-time experience was estimated from a subcohort randomly sampled from the full cohort at baseline [27].

The present study is based on a follow-up of 17.3 years (September 1986 to December 2003). In our analysis, we excluded all cases with prevalent cancer other than skin cancer at baseline. We also excluded subjects with incomplete or inconsistent dietary data, according to criteria described previously [28], and subjects with missing information on tea consumption (Figure 5.1). Our study included 3,362 incident PCa cases and 2,128 subcohort members.

The NLCS has been approved by the institutional review boards of the TNO Nutrition and Food Research Institute (Zeist, the Netherlands) and Maastricht University (Maastricht, the Netherlands). 
The Netherlands Cohort Study

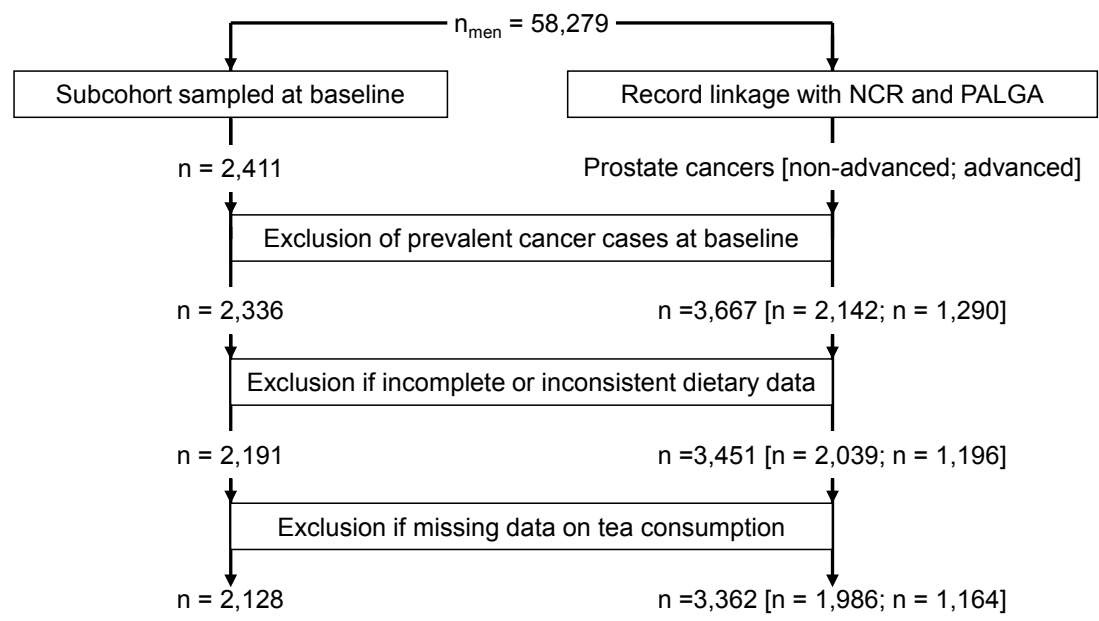

Figure 5.1

Flow diagram of male subcohort members and prostate cancer cases of the Netherlands Cohort Study, 1986-2003

Abbreviations: NCR, Netherlands cancer registry; PALGA, Netherlands nationwide pathology registry.

${ }^{a}$ Non-advanced prostate cancers were stage T1-2 and NO and M0 at diagnosis. Advanced prostate cancers were stage $\mathrm{T} 3-4, \mathrm{~N}+$, or $\mathrm{M} 1$ at diagnosis.

\section{Classification of prostate cancer cases}

We evaluated PCa subgroups based on disease stage. Advanced prostate cancers (International Union Against cancer (UICC) [29] stage III/IV) included tumors with stage T3-4, N+, or M1 at diagnosis. Stage IV (UICC) prostate cancers were a subset of advanced prostate cancers and included tumors with stage $\mathrm{T} 4, \mathrm{~N}+$, or $\mathrm{M} 1$ at diagnosis. Prostate cancers that were not identified as advanced cancers were classified as nonadvanced prostate cancers (UICC stage I/II; stage T1-2 and NO and MO at diagnosis). Our study included 1,164 advanced cancers and 1,986 non-advanced cancers (Figure 5.1). Of all advanced prostate cancers, 736 were stage IV prostate cancers. A small number of cases had missing data on tumor stage $(n=212)$ and could not be classified as either advanced or non-advanced PCa.

\section{Exposure assessment}

Dietary intake of study participants were derived from a baseline 150-item semiquantitative food frequency questionnaire (FFQ) that is described in greater detail elsewhere [24, 28, 30]. This dietary assessment was part of a larger questionnaire that also included questions about lifestyle and health.

Flavonoid contents in foods and beverages were derived from food composition tables based on data gathered in the Netherlands [31-34]. Major flavonoid subgroups 
in our study are catechin and flavonol. Specific catechins in our study are $(+)$-catechin and (-)-epicatechin. The catechins (+)-gallocatechin, (-)-epigallocatechin, (-)epicatechin gallate, and (-)-epigallocatechin gallate are found almost exclusively in tea and are therefore not evaluated separately. Specific flavonols in our study are quercetin, kaempferol, and myricetin. Flavones are another flavonoid subclass and the only flavone consumed in our population is luteolin. Since flavones are a minor group of flavonoids compared with the flavonols, the term flavonols will be used here for the sum of both groups.

With regard to tea consumption, cohort members were asked whether they drank tea and, if so, how many cups per day. The type of tea was not specified but this population rarely drank tea other than black tea [35]. Based on a pilot study, the standard cup size was assumed to be $125 \mathrm{ml}$ [35].

\section{Statistical analyses}

We used Cox proportional hazards regression to obtain hazard ratios (HRs) and corresponding 95\% confidence intervals ( $\mathrm{Cls}$ ) for the association between quartiles of flavonoid intake and categories of black tea consumption and risk of PCa and PCa subgroups based on disease stage. Cut-off points for each quartile were based on the distribution of each variable in the subcohort. Tests for linear trend were carried out by taking the median values of all quartiles and modeling intake as a continuous variable. All models were adjusted for age at baseline as a continuous variable (years). The following factors, which are hypothesized or known to be associated with either $\mathrm{PCa}$, flavonoid intake, or black tea consumption, were assessed as potential confounding variables: family history of PCa among first degree relatives (yes/no), smoking status (never, former, current), duration of smoking (years), frequency of smoking (cigarettes/day), non-occupational physical activity $(\leq 30,>30-60,>60-90,>90$ minutes/day), BMI $(<23,23-<25,25-<27,27-<30, \geq 30)$, height $(\mathrm{cm})$, diabetes (type I or II, yes/no), education level (primary school, lower vocational, high school, higher vocational/university), and intake of energy (kcal/day), alcohol (g/day), calcium (g/day), lycopene (mg/day), vitamin $\mathrm{E}(\mathrm{mg} /$ day), red meat $(\mathrm{g} / \mathrm{day})$, and coffee (cups/day). We evaluated whether adding any of these variables to the age-adjusted model changed the hazard ratio by at least $10 \%$. Because none did so, our final model was adjusted for age at baseline only. We additionally present our primary findings adjusted for all these potential confounders to show that addition of those factors did not appreciably alter the results. The proportional hazards assumption was tested using the scaled Schoenfeld residuals [36]. Standard errors were estimated by using the robust Huber-White sandwich estimator, to account for additional variance introduced by sampling from the cohort [37]. 
To examine the influence of pre-clinical disease on our results, we repeated the analyses while excluding the first two years of follow-up. To investigate possible confounding due to differences in PSA testing, we stratified by time period to determine whether the observed associations were different in an early period when PSA testing was not yet introduced in the Netherlands (1986-1994) and a late period when PSA testing was used in clinical practice (1995-2003) in the Netherlands.

All P-values were two-sided, with a P-value less than 0.05 considered to be statistically significant. Analyses were performed using STATA software (release 11, STATA Corporation, College Station, TX).

\section{Results}

The baseline characteristics of our study population are shown in Table 5.1. Compared with subcohort members, PCa cases were more likely to have a first degree relative affected with PCa and less likely to have a history of diabetes.

Important sources of flavonoids in our population are black tea, allium vegetables, apples and pears, cabbages, chocolate, legumes, and leafy vegetables (Table 5.2). Black tea is by far the major source of total catechin (80\%), kaempferol (70\%), and myricetin (78\%) and contributed significantly to intake of catechin (35\%), epicatechin (46\%), total flavonol (46\%), and quercetin (35\%).

We present age-adjusted hazard ratios (HRs) and 95\% confidence intervals (Cls). Dietary flavonoid intake was not associated with overall or non-advanced PCa risk (Table 5.3). Intake of total catechin, epicatechin, kaempferol, and myricetin was associated with a decreased risk of stage III/IV or IV PCa and hazard ratios of stage IV PCa for the highest versus lowest quartile of intake of total catechin, epicatechin, kaempferol, and myricetin were respectively $0.73(95 \% \mathrm{Cl}: 0.57,0.95), 0.74(95 \% \mathrm{Cl}$ : $0.57,0.95), 0.78$ (95\% Cl: $0.61,1.00)$, and 0.71 (95\% Cl: $0.55,0.91)$.

We investigated associations between black tea consumption and PCa risk. The median consumption of black tea in our population is 2 cups per day. Black tea consumption was inversely associated with risk of advanced (stage III/IV) and stage IV PCa but not with risk of overall and non-advanced (stage I/II) PCa (Table 5.4). The HR of advanced PCa for the highest versus the lowest category of black tea consumption ( $\geq 5$ versus $\leq 1$ cups/day) was 0.75 (95\% Cl: $0.59,0.97)$ and the $H R$ for an increment of 1 cup per day was 0.97 (95\% Cl: $0.93,1.01)$. The HR of stage IV PCa for the highest versus the lowest category of black tea consumption ( $\geq 5$ versus $\leq 1$ cups/day) was 0.67 ( $95 \% \mathrm{Cl}$ : $0.50,0.91)$ and the HR for an increment of 1 cup per day was $0.95(95 \% \mathrm{Cl}: 0.90,0.99)$. These associations were similar when adjusted for all potential confounders (HR of stage IV PCa for $\geq 5$ versus $\leq 1$ cups/day: 0.67 (95\% Cl: 0.48, 0.94)). Fruits and vegetables contain many antioxidant nutrients other than flavonoids (e.g. vitamin C, lycopene). 
We studied whether the association between black tea consumption and stage III/IV and stage IV PCa was modified by intake of total fruits and vegetables ( $\leq$ median intake, $>$ median intake), but it was not ( $P$ for interaction $>0.05$ ).

To examine the influence of preclinical disease on our results, we repeated the analyses while excluding the first two years of follow-up, and we observed that the results were unchanged. To examine whether confounding by PSA testing might explain the associations, we stratified by time period and separately evaluated an early period (pre-PSA period: 1986-1994) and a late period (PSA period: 1995-2003). We observed that the point estimates were comparable in both time periods and HRs for the association between black tea consumption ( $\geq 5$ versus $\leq 1 \mathrm{cups} /$ day) and stage IV PCa risk in the early and late period were $0.63(95 \% \mathrm{Cl}: 0.41,0.97)$ and $0.69(95 \% \mathrm{Cl}$ : $0.48,1.00)$, respectively. 


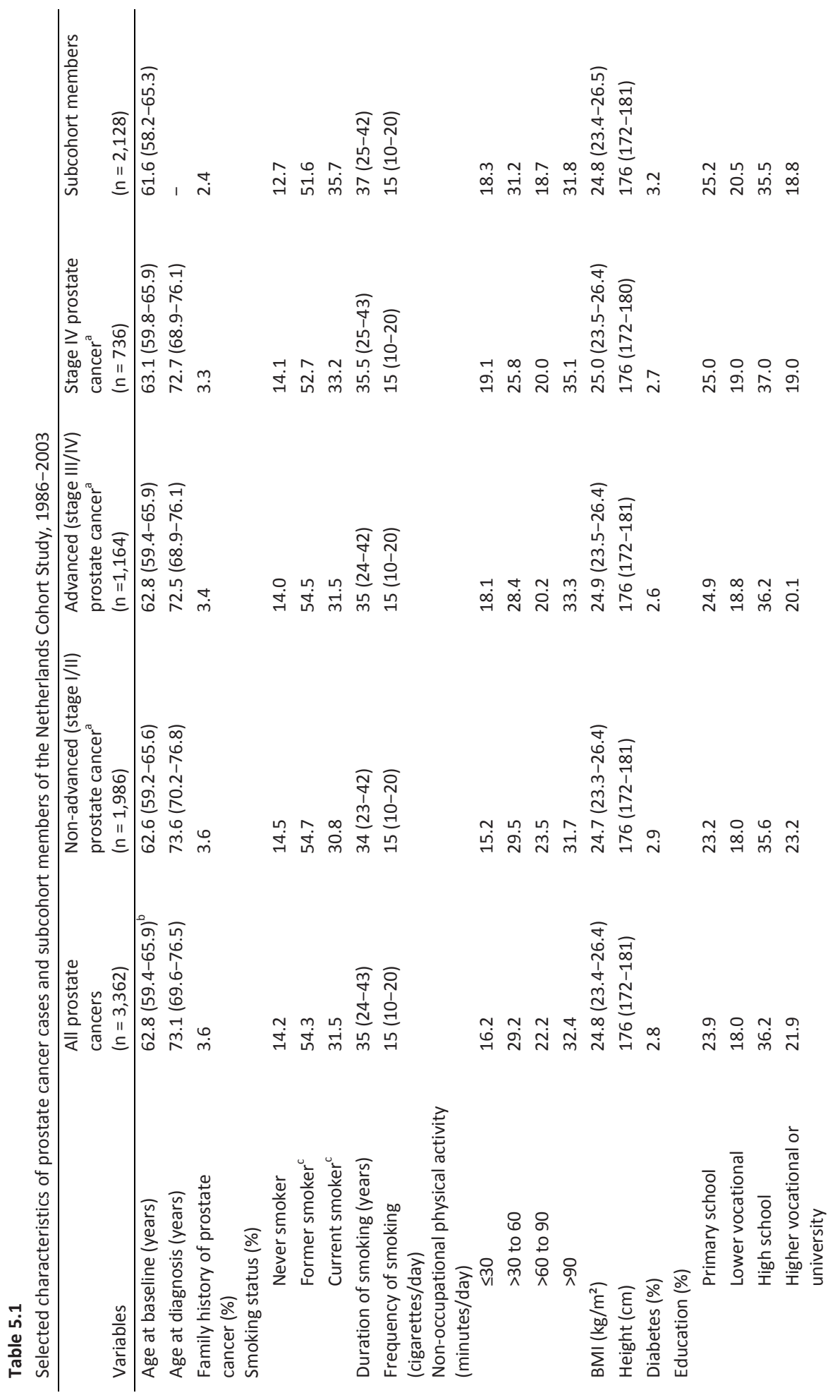




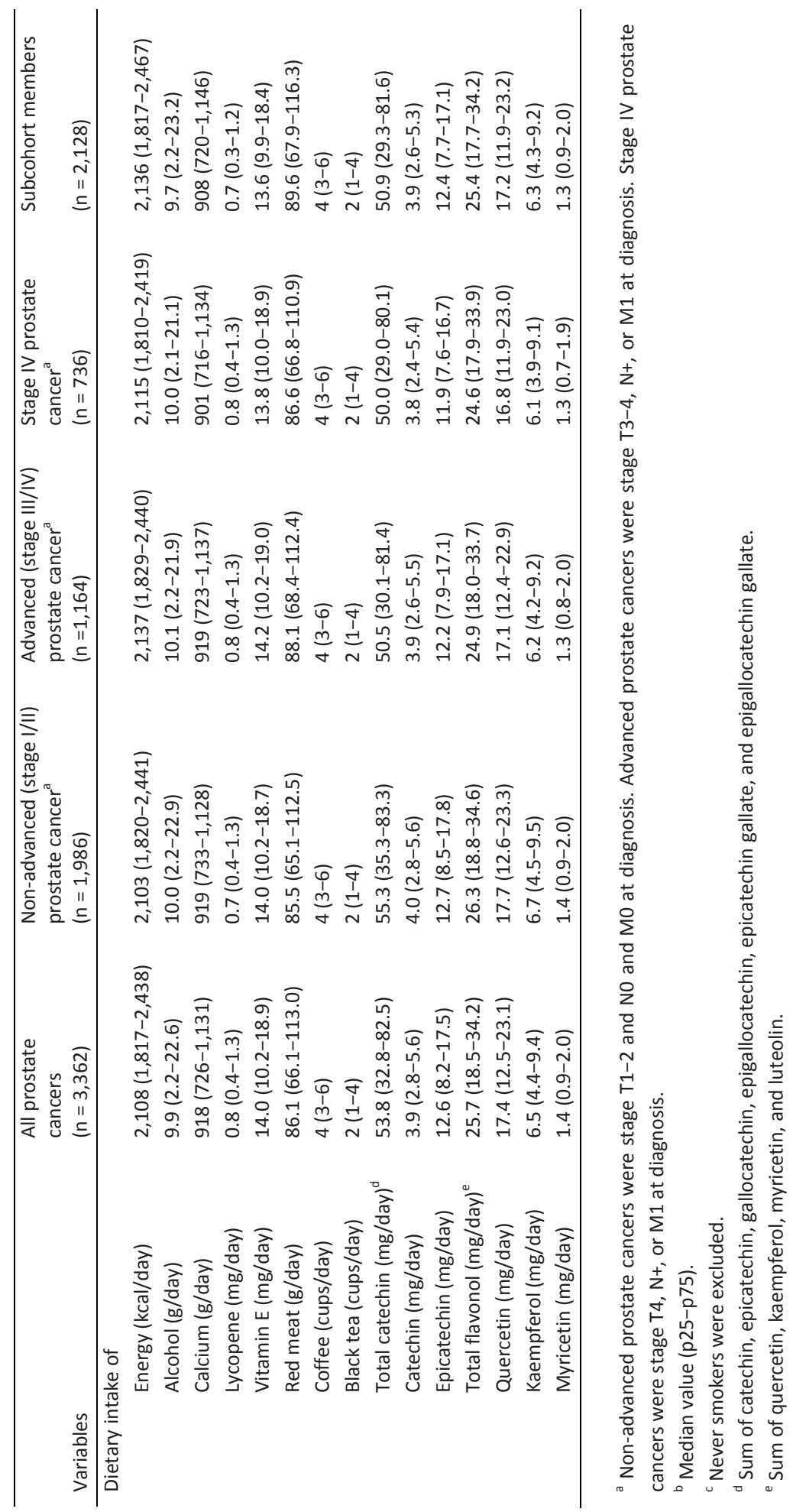




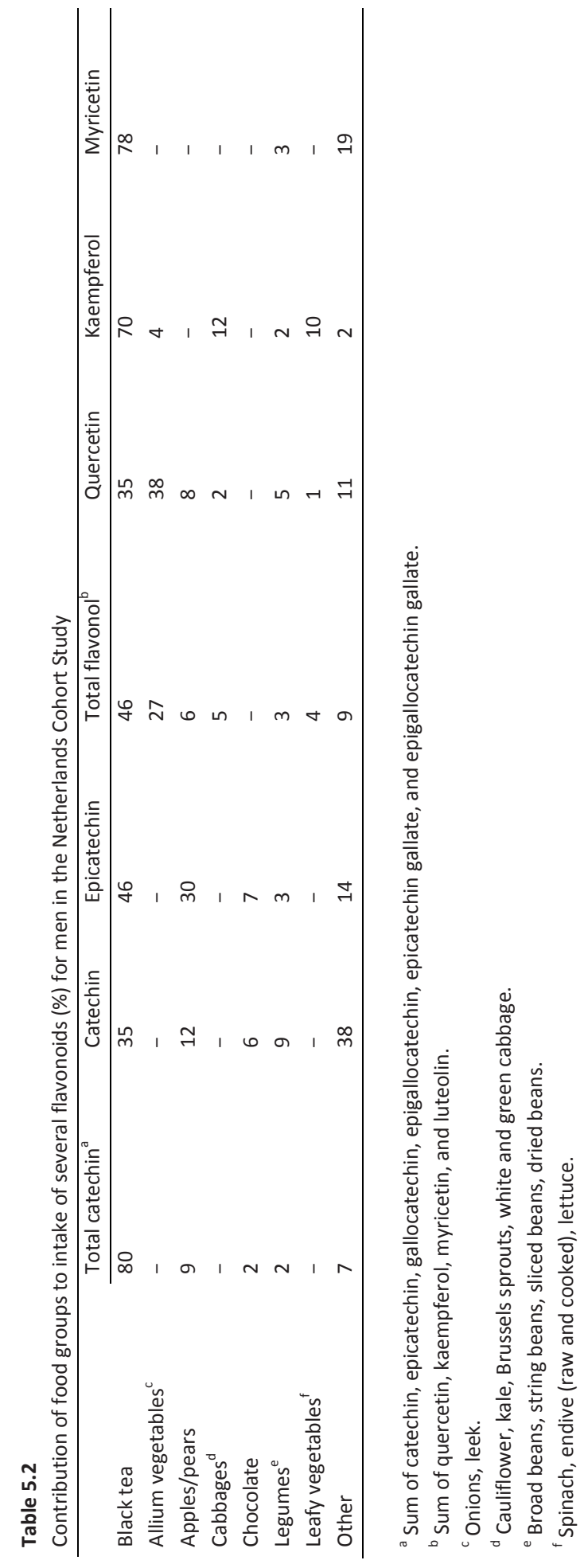




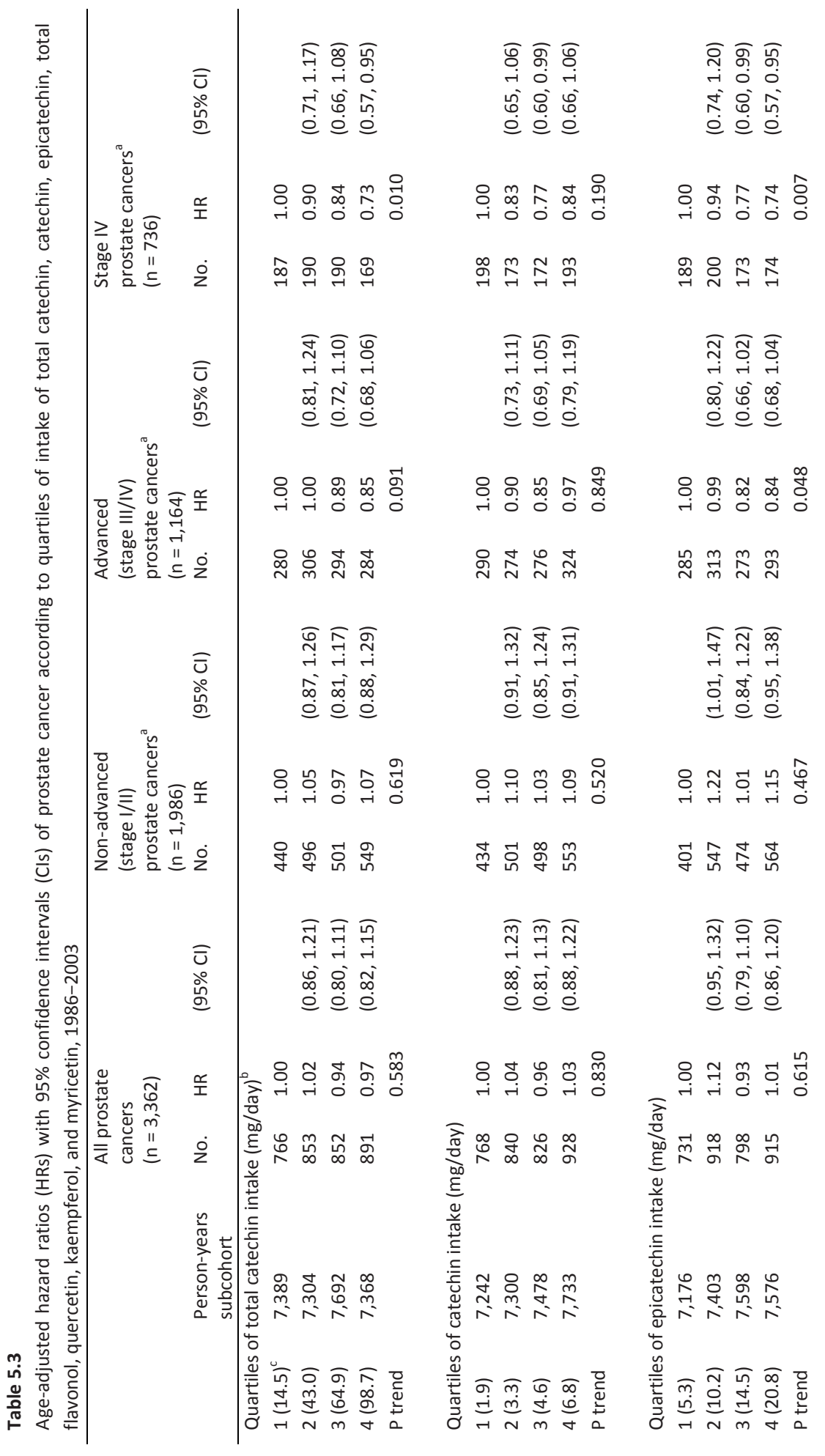




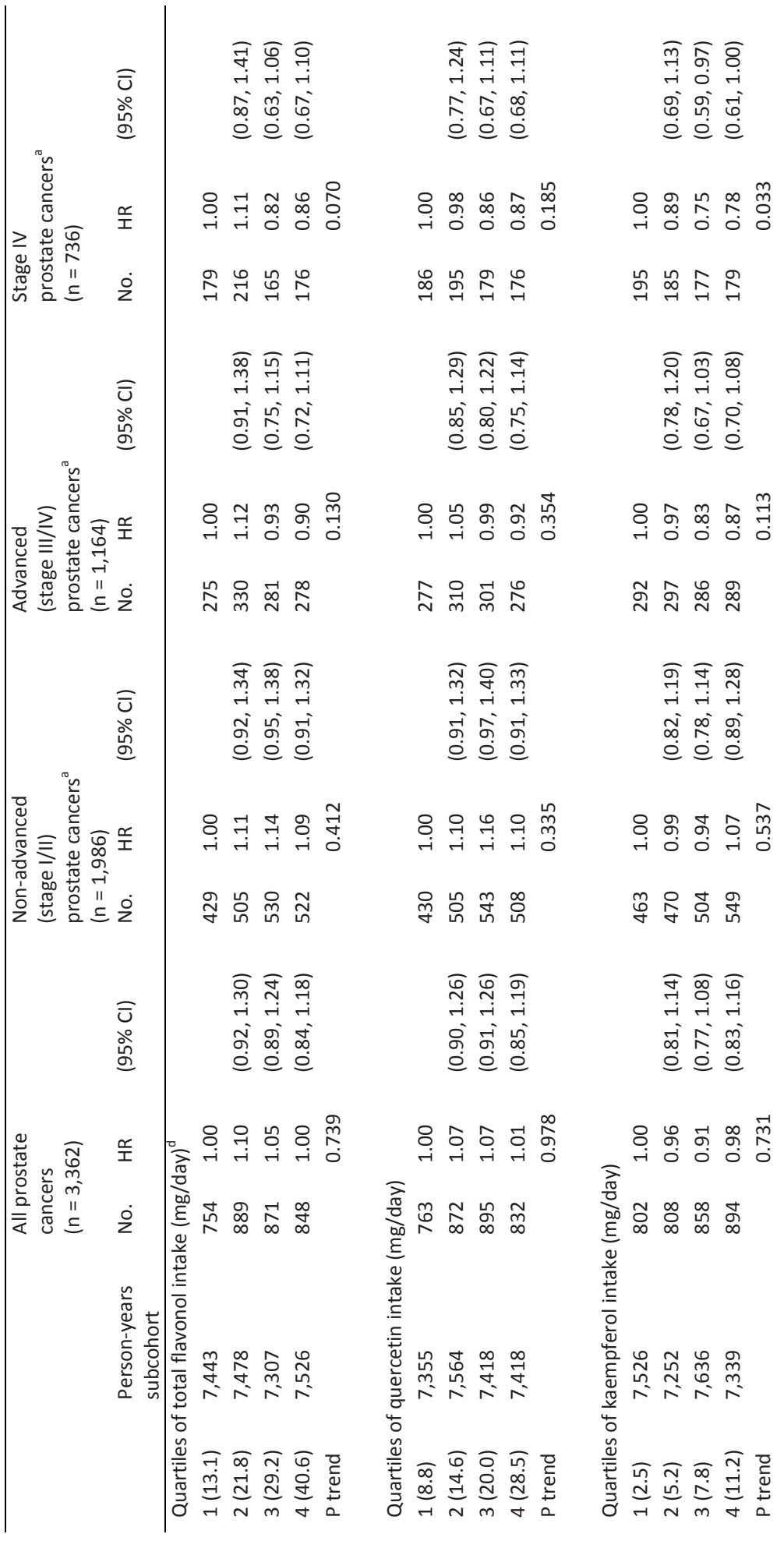




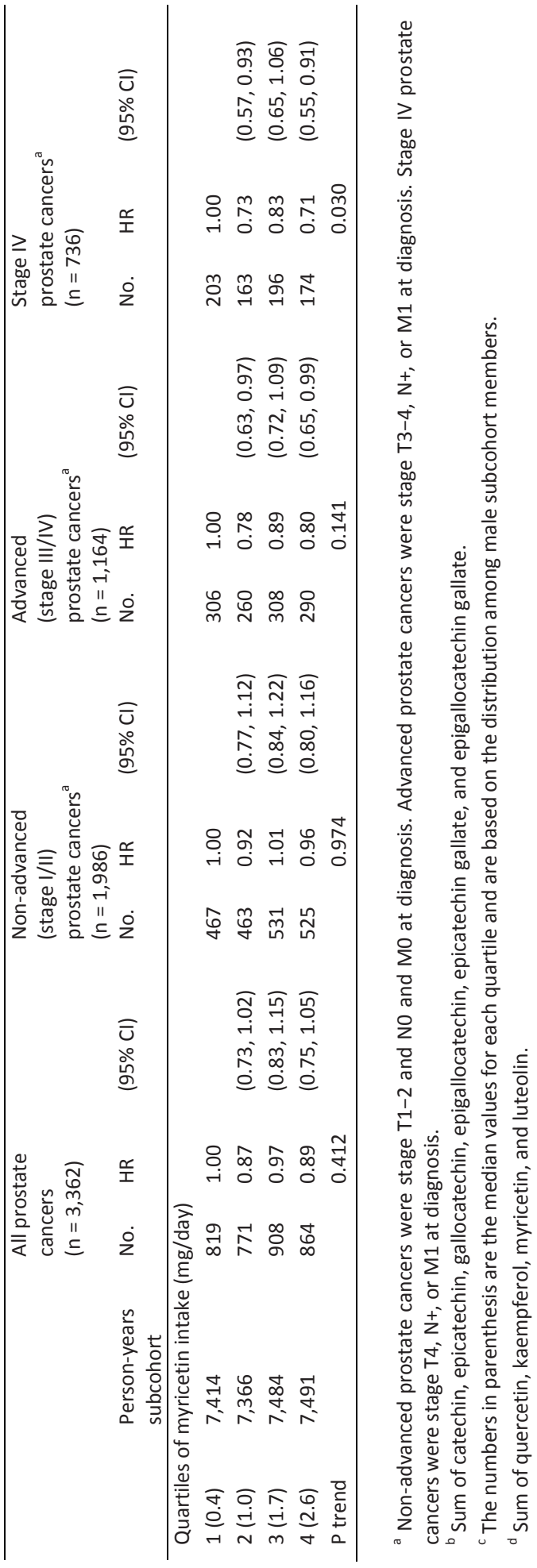




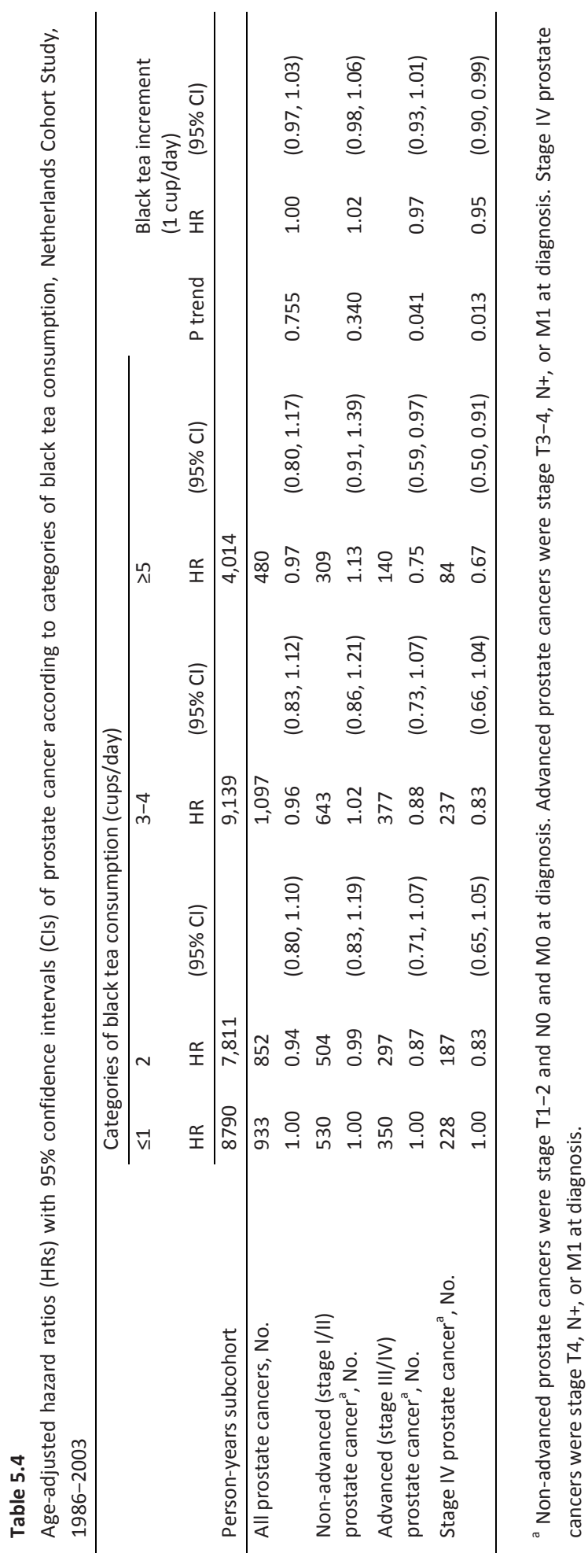




\section{Discussion}

This large prospective study showed that intake of total catechin, epicatechin, kaempferol, and myricetin and consumption of black tea were associated with a decreased risk of PCa of advanced stage (stage III/IV and IV). None of the exposures considered were associated with risk of overall or non-advanced (stage I/II) PCa. These associations were robust in that they remained unchanged after exclusion of cases diagnosed during the first two years of follow-up. The HRs were similar after multivariable adjustment, which implies that there was no substantial confounding by any of the additionally included covariables.

Only few observational studies investigated associations between flavonoid intake and PCa risk and most of these reported null results [15-20]. None of these studies evaluated PCa subgroups based on tumor characteristics. One small Finish prospective cohort study, which included 95 PCa cases, previously showed that myricetin intake was inversely associated with overall PCa risk [17]. In our population, black tea was by far the major source of myricetin (78\%), but in the Finish study information on tea consumption was not available because tea consumption in Finland is very low. The major source of myricetin in the Finish population was berries.

A recent meta-analysis of PCa and consumption of black tea (11 studies) and green tea (seven studies) showed that green tea but not black tea may have a protective effect on PCa [21]. Most studies of green tea were conducted in Asian populations. Previous observational studies of black tea consumption and PCa mostly reported null results $[5,21]$, but interestingly, only two small-scale studies considered the pathologic manifestations of PCa. One population-based case-control study (87 aggressive PCa cases) [38] and one prospective cohort study (37 Gleason $\geq 8$ prostate cancers) [22] observed no association between black tea consumption and risk of aggressive PCa. One prospective cohort study in Japan previously showed that consumption of green tea was inversely associated with risk of advanced PCa but not overall or localized PCa [39]. This study included 404 incident prostate cancers of which 114 were advanced cancers. The multivariable adjusted relative risk for advanced PCa for the highest versus the lowest category of green tea consumption ( $\geq 5$ versus < $1 \mathrm{cups} /$ day) was $0.52(95 \% \mathrm{Cl}: 0.28,0.96$; $\mathrm{P}$ for trend $=0.01)$. This association was stronger than the association between black tea consumption and advanced PCa risk in our study and one possible explanation for this is the higher concentration of catechins in green compared to black tea [7].

Tea polyphenols are mainly absorbed from the small intestine and are then subject to phase II metabolism, which likely affects their bioavailability [7]. A human pre-prostatectomy trial, however, showed that black tea and green polyphenols are bio-available in the prostate [40]. Flavonoids have various biological properties that 
can explain the association with a decreased risk of advanced stage PCa. The most widely recognized biological properties of flavonoids are the antioxidant properties [2, $41]$, and oxidative stress is believed to be related to prostate carcinogenesis $[42,43]$. Various antioxidants have been associated with advanced stage PCa [44, 45]. Besides that, flavonoids can affect apoptosis, angiogenesis, and inflammatory processes all of which play roles in PCa progression [6-11].

Black tea contains a high concentration of theaflavins and thearubigins, which are polymeric polyphenols. These compounds are formed from catechins during fermentation of tea leaves and might also have anticarcinogenic activity. There are studies using cell lines that showed that theaflavin and thearubigin inhibit PCa growth $[46,47]$. However, because of the limited bioavailability of these compounds it is believed that they generally do not contribute to the chemopreventive effects of black tea in vivo [7].

In our study we did not obtain information on infusion time of tea or tea strength. Such additional information could have provided us with a more precise estimation of daily flavonoid intake. Another possible limitation is that misclassification may have occurred in the assignment of flavonoid levels to the foods reported. Flavonoid contents are known to vary greatly by variety, thus individual preferences for particular varieties of foods would lead to misclassification. Our analyses were performed by using baseline FFQ data and it is thus possible that changes in black tea consumption after 1986 resulted in attenuation of our risk estimates. Inaccuracy of recall of tea consumption as such also may be a source of bias. Unfortunately, we had no separate data on tea available from the validation study of our food-frequency questionnaire [28], but considering the simplicity of the question on tea and the small number of blanks encountered, we would expect that the accuracy of recall was even better than average. Another limitation is that we had no information on PSA testing, which might be a confounder. We however showed that the observed associations were similar in an early period when PSA testing was not yet introduced in the Netherlands (1986-1994) and a late period when PSA testing was used in clinical practice in the Netherlands (1995-2003). Besides that, it is important to note that in the Netherlands PSA testing was introduced relatively slowly in comparison to other high-income countries and has never been used for systematic screening or case finding [48]. In 2001 in the Netherlands only 14\% of men over 45 years of age had a PSA measurement in the previous 5 years [48]. Based on this evidence it is unlikely that PSA testing is an explanation for the observed associations in our study.

Our study has several strengths including its prospective design, large sample size, and long and nearly complete follow-up. The range of black tea intake in our cohort was wide. We had data on tumor stage for most cases, which made it possible to stratify by disease stage, and our study included a large number of advanced 
prostate cancers. Our FFQ contained questions about all important sources of flavonoids and we have data on many potential confounding variables.

In conclusion, men with a high intake of black tea, total catechin, epicatechin, kaempferol, and myricetin had a reduced risk of PCa of advanced stage. These findings need to be reproduced in other large-scale observational studies with comprehensive data on flavonoid contents in foods and beverages and evaluation of PCa subgroups based on pathologic manifestations.

\section{References}

1. Arts IC, Hollman PC. Polyphenols and disease risk in epidemiologic studies. The American journal of clinical nutrition 2005;81(1 Suppl):317s-325s.

2. Pietta PG. Flavonoids as antioxidants. Journal of natural products 2000;63(7):1035-1042.

3. Arts IC, Hollman PC, Feskens EJ, et al. Catechin intake and associated dietary and lifestyle factors in a representative sample of Dutch men and women. Eur J Clin Nutr 2001;55(2):76-81.

4. Hertog MG, Hollman PC, Katan MB, et al. Intake of potentially anticarcinogenic flavonoids and their determinants in adults in The Netherlands. Nutr Cancer 1993;20(1):21-29.

5. Yuan JM, Sun C, Butler LM. Tea and cancer prevention: Epidemiological studies. Pharmacol Res 2011;64(2):123-135.

6. Johnson JJ, Bailey HH, Mukhtar H. Green tea polyphenols for prostate cancer chemoprevention: a translational perspective. Phytomedicine 2010;17(1):3-13.

7. Henning SM, Wang P, Heber D. Chemopreventive effects of tea in prostate cancer: Green tea versus black tea. Molecular nutrition \& food research 2011;55(6):905-920.

8. Yang CS, Wang H, Li GX, et al. Cancer prevention by tea: Evidence from laboratory studies. Pharmacol Res 2011;64(2):113-122.

9. Garcia-Lafuente A, Guillamon E, Villares A, et al. Flavonoids as anti-inflammatory agents: implications in cancer and cardiovascular disease. Inflamm Res 2009;58(9):537-552.

10. Moon YJ, Wang X, Morris ME. Dietary flavonoids: effects on xenobiotic and carcinogen metabolism. Toxicol In Vitro 2006;20(2):187-210.

11. Yang CS, Lambert JD, Sang S. Antioxidative and anti-carcinogenic activities of tea polyphenols. Archives of toxicology 2009;83(1):11-21.

12. Bettuzzi S, Brausi M, Rizzi F, et al. Chemoprevention of human prostate cancer by oral administration of green tea catechins in volunteers with high-grade prostate intraepithelial neoplasia: a preliminary report from a one-year proof-of-principle study. Cancer research 2006;66(2):1234-1240.

13. Brausi M, Rizzi F, Bettuzzi S. Chemoprevention of human prostate cancer by green tea catechins: two years later. A follow-up update. European urology 2008;54(2):472-473.

14. Montironi R, Mazzucchelli R, Lopez-Beltran A, et al. Prostatic intraepithelial neoplasia: its morphological and molecular diagnosis and clinical significance. BJU international;108(9):1394-1401.

15. Bosetti C, Bravi F, Talamini R, et al. Flavonoids and prostate cancer risk: a study in Italy. Nutrition and cancer 2006;56(2):123-127.

16. Knekt $P$, Jarvinen $R$, Seppanen $R$, et al. Dietary flavonoids and the risk of lung cancer and other malignant neoplasms. American journal of epidemiology 1997;146(3):223-230.

17. Knekt $P$, Kumpulainen J, Jarvinen $R$, et al. Flavonoid intake and risk of chronic diseases. The American journal of clinical nutrition 2002;76(3):560-568.

18. Mursu J, Nurmi T, Tuomainen TP, et al. Intake of flavonoids and risk of cancer in Finnish men: The Kuopio Ischaemic Heart Disease Risk Factor Study. International journal of cancer 2008;123(3):660-663.

19. Strom SS, Yamamura Y, Duphorne CM, et al. Phytoestrogen intake and prostate cancer: a case-control study using a new database. Nutrition and cancer 1999;33(1):20-25.

20. Hirvonen $\mathrm{T}$, Virtamo J, Korhonen $\mathrm{P}$, et al. Flavonol and flavone intake and the risk of cancer in male smokers (Finland). Cancer Causes Control 2001;12(9):789-796.

21. Zheng J, Yang B, Huang T, et al. Green tea and black tea consumption and prostate cancer risk: an exploratory meta-analysis of observational studies. Nutrition and cancer 2011;63(5):663-672. 
22. Shafique K, McLoone P, Qureshi K, et al. Tea Consumption and the Risk of Overall and Grade Specific Prostate Cancer: A Large Prospective Cohort Study of Scottish Men. Nutr Cancer 2012.

23. Giovannucci E, Liu Y, Platz EA, et al. Risk factors for prostate cancer incidence and progression in the health professionals follow-up study. Int J Cancer 2007;121(7):1571-1578.

24. van den Brandt PA, Goldbohm RA, van 't Veer $P$, et al. A large-scale prospective cohort study on diet and cancer in The Netherlands. Journal of clinical epidemiology 1990;43(3):285-295.

25. van den Brandt PA, Schouten LJ, Goldbohm RA, et al. Development of a record linkage protocol for use in the Dutch Cancer Registry for Epidemiological Research. International journal of epidemiology 1990;19(3):553-558.

26. Goldbohm R, van den Brandt $P$, Dorant $E$. Estimation of the coverage of Dutch municipalities by cancer registries and PALGA based on hospital discharge data. Tijdschr Soc Gezondheidsz 1994(72):80-84.

27. Barlow WE, Ichikawa L, Rosner D, et al. Analysis of case-cohort designs. Journal of clinical epidemiology 1999;52(12):1165-1172.

28. Goldbohm RA, van den Brandt PA, Brants HA, et al. Validation of a dietary questionnaire used in a largescale prospective cohort study on diet and cancer. European journal of clinical nutrition 1994;48(4):253265.

29. Sobin L, Gospodarowicz M, Wittekind C, et al. TNM Classification of Malignant Tumors. 7th ed. Oxford, UK: Wiley-Blackwell; 2009.

30. Goldbohm RA, van 't Veer P, van den Brandt PA, et al. Reproducibility of a food frequency questionnaire and stability of dietary habits determined from five annually repeated measurements. European journal of clinical nutrition 1995;49(6):420-429.

31. Arts IC, van De Putte B, Hollman PC. Catechin contents of foods commonly consumed in The Netherlands. 2. Tea, wine, fruit juices, and chocolate milk. J Agric Food Chem 2000;48(5):1752-1757.

32. Arts IC, van de Putte B, Hollman PC. Catechin contents of foods commonly consumed in The Netherlands. 1. Fruits, vegetables, staple foods, and processed foods. J Agric Food Chem 2000;48(5):1746-1751.

33. Hertog MGL, Hollman PCH, Katan MB. Content of potentially anticarcinogenic flavonoids of 28 vegetables and 9 fruits commonly consumed in the Netherlands. J Agric Food Chem 1992;48:1746-1751.

34. Hertog MHL, Hollman PCH, van de Putte B. Content of potentially anticarcinogenic flavonoids in tea infusions, wines and fruit juices. J Agric Food Chem 1993;41:1242-1246.

35. Goldbohm RA, Hertog MG, Brants HA, et al. Consumption of black tea and cancer risk: a prospective cohort study. Journal of the National Cancer Institute 1996;88(2):93-100.

36. Schoenfeld D. Partial residuals for the proportional hazards regression model. Biometrika 1982;1:239241.

37. Lin DY, Wei LY. The robust inference for the Cox proportional hazards model. J Am Stat Assoc 1989;84:1074-1078.

38. Slattery ML, West DW. Smoking, alcohol, coffee, tea, caffeine, and theobromine: risk of prostate cancer in Utah (United States). Cancer Causes Control 1993;4(6):559-563.

39. Kurahashi N, Sasazuki S, Iwasaki M, et al. Green tea consumption and prostate cancer risk in Japanese men: a prospective study. American journal of epidemiology 2008;167(1):71-77.

40. Henning SM, Aronson W, Niu Y, et al. Tea polyphenols and theaflavins are present in prostate tissue of humans and mice after green and black tea consumption. J Nutr 2006;136(7):1839-1843.

41. Higdon JV, Frei B. Tea catechins and polyphenols: health effects, metabolism, and antioxidant functions. Critical reviews in food science and nutrition 2003;43(1):89-143.

42. Halliwell B. Oxidative stress and cancer: have we moved forward? The Biochemical journal 2007;401(1):1-11.

43. Khandrika L, Kumar B, Koul S, et al. Oxidative stress in prostate cancer. Cancer letters 2009;282(2):125136.

44. Wang $P$, Heber D, Henning SM. Quercetin increased the antiproliferative activity of green tea polyphenol (-)-epigallocatechin gallate in prostate cancer cells. Nutr Cancer 2012;64(4):580-587.

45. Henning SM, Wang P, Heber D. Chemopreventive effects of tea in prostate cancer: green tea versus black tea. Mol Nutr Food Res 2011;55(6):905-920.

46. Kalra N, Seth K, Prasad S, et al. Theaflavins induced apoptosis of LNCaP cells is mediated through induction of p53, down-regulation of NF-kappa B and mitogen-activated protein kinases pathways. Life sciences 2007;80(23):2137-2146. 
47. Sakamoto K. Synergistic effects of thearubigin and genistein on human prostate tumor cell (PC-3) growth via cell cycle arrest. Cancer letters 2000;151(1):103-109.

48. Cremers RG, Karim-Kos HE, Houterman S, et al. Prostate cancer: trends in incidence, survival and mortality in the Netherlands, 1989-2006. Eur J Cancer 2010;46(11):2077-2087. 
Chapter 6: Oxidative Stress-Related Genetic Variants,

Pro- and Antioxidant Intake and Status, and Advanced Prostate Cancer Risk

Milan S. Geybels Piet A. van den Brandt Frederik J. van Schooten Bas A.J. Verhage Submitted 


\begin{abstract}
Background

Increased oxidative stress has been linked to prostate cancer (PCa). We investigated oxidative stress-related genetic variants in relation to advanced PCa risk and examined potential interactions with pro- and antioxidant exposures.
\end{abstract}

\title{
Methods
}

A case-cohort analysis was conducted in the Netherlands Cohort Study, which included 58,279 men aged 55-69 years. Cohort members completed a baseline questionnaire and provided toenail clippings, which were used to determine selenium levels and to isolate DNA for genotyping. Incident advanced PCa cases, defined on the basis of TNM stage at diagnosis, were identified during 17.3 years of follow-up. The analysis included 14 oxidative stress-related gene variants and 11 pro- and antioxidant dietary and lifestyle factors. Cox regression models were used and false discovery rate (FDR) was applied to adjust for multiple testing.

\section{Results}

Complete genotyping data were available for 952 advanced (stage III and IV) PCa cases and 1,798 subcohort members. CAT rs1001179 was associated with stage III/IV and stage IV PCa risk, with HRs per minor allele of 1.16 (95\% C: 1.01, 1.33) and 1.25 (95\% $\mathrm{Cl}: 1.07,1.46)$, respectively. We tested 151 gene-environment interactions in relation to both stage III/IV and IV PCa risk. Seven interactions were statistically significant after adjusting for multiple testing (FDR Q-value <0.20); for stage III/IV PCa these involved intake of $\beta$-carotene (GPX1 rs17650792, hOGG1 rs1052133) and heme iron (GPX1 rs1800668 and rs3448), and for stage IV PCa these involved intake of catechin (SOD2 rs4880) and heme iron (hOGG1 rs1052133, SOD1 rs10432782).

\section{Conclusion}

This study of advanced PCa risk showed a marginal association with a CAT polymorphism and seven novel gene-environment interactions in the oxidative stress pathway. 


\section{Introduction}

Oxidative stress results from an imbalance between antioxidant protection and reactive oxygen species (ROS) produced by pro-oxidants [1-3]. ROS are strong oxidizing agents that cause damage to cellular constituents when levels are elevated $[1,2]$. Antioxidants include endogenous enzymes (e.g., superoxide dismutase, SOD; catalase, CAT; and glutathione peroxidase; GPX) and exogenous dietary nutrients (e.g., vitamins $C$ and $E$, carotenoids, flavonoids, selenium) [4, 5]. Important pro-oxidant factors include inflammatory processes and the mitochondrial electron transport chain, but also exogenous exposures such as cigarette smoking, dietary iron, and radiation [2, 4]. Oxidative stress becomes more common with advancing age $[6,7]$, and has been implicated in age-related degenerative diseases, including prostate cancer (PCa) [8-11].

Previous epidemiological studies on antioxidant nutrients and PCa risk have generated mixed results [12]. More than fifty observational (prospective and case-control) studies investigated the associations of the major dietary antioxidants vitamins $C$ and $E$, lycopene, $\beta$-carotene, and selenium. Results from these studies showed that most antioxidants were not associated with overall or advanced PCa risk $[12,13]$, with the exception of selenium, which has been inversely associated with overall and advanced PCa risk in many studies [14], including our study [15, 16]. Secondary results from two randomized controlled trials, the Alpha-Tocopherol, BetaCarotene Cancer Prevention (ATBC) study [17], and the Nutritional Prevention of Cancer (NPC) study [18], showed PCa risk reductions for vitamin E and selenium, respectively. These findings led to the design of the Selenium and Vitamin E Cancer Prevention Trial (SELECT), which however showed no protective effect of both nutrients [19]. There have been a number of observational studies that investigated pro-oxidant exposures such as smoking, alcohol, and dietary iron in relation to overall and advanced PCa risk, but these studies do not support an association [20-24].

Genetic variation in genes related to oxidative stress may influence PCa risk. These genes include those encoding pro- and antioxidant enzymes but also other oxidative stress-related genes (e.g., oxidative DNA damage repair). Several variants in these genes have been identified that have potential functional consequences [25-30]. Candidate gene-association studies investigated these genetic variants in relation to $\mathrm{PCa}$ risk and showed significant associations for variants in different genes such as SOD2, PON1, GPX1, NOS2A, NOS3, and hOGG1 [25-30]. Genome-wide association studies of $\mathrm{PCa}$, however, did not identify risk variants in major oxidative stress-related genes [31]. Besides having a main effect on PCa risk, oxidative stress-related gene variants may also interact with pro- and antioxidant exposures and potentially modify their effects. This has been investigated in a small number of studies that focused on specific a priori defined gene-environment interactions. These studies have produced 
statistically significant results, including the interactions between SOD2 and selenium [32], SOD2 and iron [20], and hOGG1 and $\alpha$-tocopherol [29].

In the present study, we evaluated 14 genetic variants in oxidative stress-related genes. The gene variants are single nucleotide polymorphisms (SNPs) reported in prior studies to have either a functional effect or an association with PCa risk. We investigated associations between these SNPs and advanced PCa risk and examined potential gene-environment interactions with major pro- and antioxidant exposures. We specifically studied advanced PCa, which is a subtype of PCa associated with a poor prognosis that is, therefore, clinically relevant [33].

\section{Methods}

Study population and design

The prospective Netherlands Cohort Study included 58,279 men, aged 55 to 69 years at baseline in September 1986. Study participants completed a baseline questionnaire on dietary habits, lifestyle, health, and several other potential cancer risk factors [34]. Approximately $81 \%$ of cohort members provided toenail clippings from all ten toes, which were used to determine toenail selenium levels and to isolate DNA for genotyping. Cancer cases were identified by annual record linkage to the Netherlands Cancer Registry and the Dutch Pathology Registry [35]. The completeness of follow-up through linkage with these registries was assessed to be at least 96\% [36]. The Netherlands Cohort Study employs the case-cohort design [37]; cases are derived from the entire cohort and the person-time experience is estimated from a subcohort randomly sampled from the full cohort at baseline $(n=2,411)$. All participants with prevalent cancer other than skin cancer at baseline were excluded (approximately $3 \%$ ). Figure 1 shows a flow diagram of cases and subcohort members. The Netherlands Cohort Study has been approved by the institutional review boards of the TNO Nutrition and Food Research Institute (Zeist, the Netherlands) and Maastricht University (Maastricht, the Netherlands). 


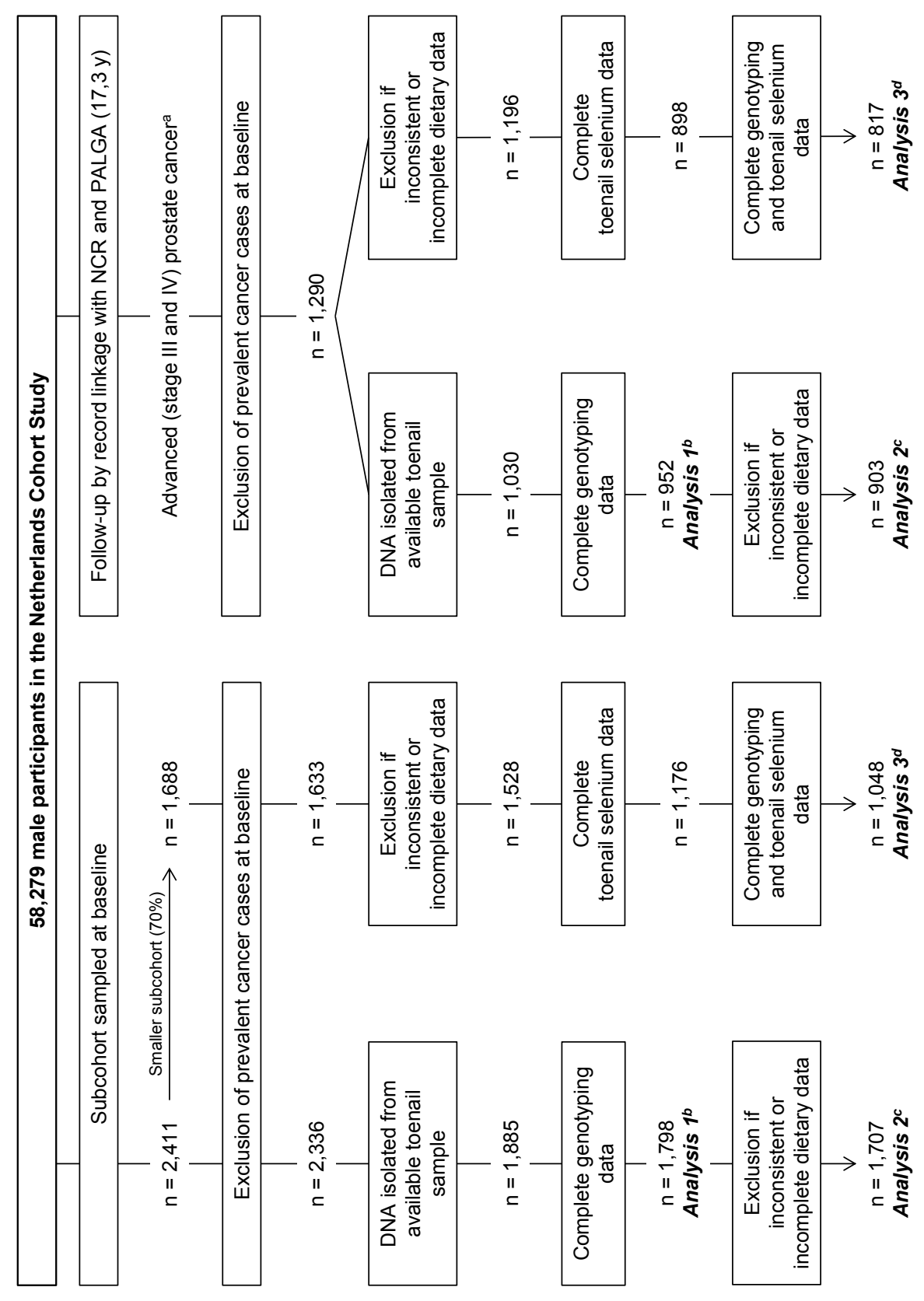




\section{Figure 6.1}

Flow diagram of advanced prostate cancer cases and subcohort members, Netherlands Cohort Study, 1986-2003

Abbreviations: NCR, Netherlands Cancer Registry; PALGA, Dutch Pathology Registry.

${ }^{a}$ Advanced prostate cancer was defined as T3-4, $\mathrm{N}+$, or $\mathrm{M} 1$ at diagnosis (International Union Against Cancer, stage III/IV). On average, $63 \%$ of advanced cases were stage IV prostate cancer (stage T4, N+, or M1 at diagnosis).

${ }^{\mathrm{b}}$ Analysis 1 is on the associations between oxidative stress-related genetic variants and advanced prostate cancer risk. The analysis included 952 cases and 1,798 subcohort members.

${ }^{c}$ Analysis 2 is on potential interactions between oxidative stress-related genetic variants and pro- and antioxidant intake on advanced prostate cancer risk. The analysis included 903 cases and 1,707 subcohort members.

${ }^{d}$ Analysis 3 is on potential interactions between oxidative stress-related genetic variants and toenail selenium levels on advanced prostate cancer risk. The analysis included 817 cases and 1,048 subcohort members. A smaller version of the subcohort (70\%) was used for this analysis.

\section{Ascertainment and classification of participants who developed prostate cancer}

During 17.3 years of follow-up (September 1986 to December 2003), 3,667 incident PCa cases (ICD-O-3 code C61) were identified. PCa cases were classified on the basis of Tumor-Node-Metastasis (TNM) staging (pathological TNM, where available, or clinical TNM) [38]. In the present analysis we specifically evaluated advanced PCa (International Union Against Cancer, stage III and IV [38]), which included tumors with T3-4, N+, or M1 at diagnosis ( $n=1,290$; Figure 1). Stage IV PCa is a subset of advanced PCa and was stage $\mathrm{T} 4, \mathrm{~N}+$, or $\mathrm{M} 1$ at diagnosis $(\mathrm{n}=817)$. A number of cases had missing data on tumor stage $(n=235)$ and were excluded from the analysis.

\section{DNA isolation, gene variant selection, and genotyping}

DNA was isolated from available toenail samples using a phenol-chloroform extraction $[27,39]$. We previously showed that toenail samples are a long-term stable source of DNA for genotyping [39]. The minimum amount of toenail material required for DNA extraction was $4 \mathrm{mg}$ and we excluded participants who provided less. Toenail DNA was successfully isolated for 1,030 cases and 1,885 subcohort members (>99.9\% successful DNA isolation; Figure 1).

We selected single nucleotide polymorphisms (SNPs) in oxidative stress-related genes reported in prior studies to have a functional effect (e.g., altered gene expression or enzyme activity) or an association with PCa risk. The following SNPs were selected: CAT rs1001179 [40], GPX1 rs17650792 [27], GPX1 rs1800668 [27], GPX1 rs3448 [41], hOGG1 rs1052133 [29], NOS2A rs2297518 [28], NOS2A rs9282801 [28], NOS3A rs1799983 [28], NQ1 rs1800566 [42], PON1 rs662 [26], PON1 rs854560 [26], SOD1 rs10432782 [43], SOD2 rs4816407 [43], and SOD2 rs4880 [25]. Selection of genetic variants was restricted to those with a minor allele frequency of at least $10 \%$. 
MPO rs2333227 was not included in the final SNP selection because it was not possible to design unique primers for the SNP given the other SNPs selected in our study.

SNP genotyping was done by designing polymerase chain reaction (PCR) assays and extension primers with the use of MassARRAY software, version 4.0 (Sequenom Inc., Hamburg, Germany). PCR and extension reactions were performed according to the manufacturer's instructions, and extension product sizes were determined by mass spectrometry with the use of the iPLEX Gold system (Sequenom Inc., Hamburg, Germany).

Quality control included genotyping of blind duplicate samples ( $n=143$, or $5 \%$ of all genotyped samples), which revealed $>99 \%$ agreement on genotyping calls across all SNPs assayed. Samples with a sample call rate (i.e. percentage of called SNPs per sample) of less than $95 \%$ were excluded ( 78 cases and 87 subcohort members) and 952 cases and 1,798 subcohort members had complete genotyping data (Figure 6.1). All SNPs had $>99 \%$ genotype completion rates (i.e. percentage of non-missing samples per SNP). None of the SNPs violated Hardy-Weinberg equilibrium $(P>0.05)$.

\section{Exposure assessment}

A baseline food frequency questionnaire (FFQ) was used to assess dietary pro- and antioxidant intake $[44,45]$. The FFQ was part of a larger questionnaire that also included questions regarding various other factors such as lifestyle, family history of cancer, and health. We selected and studied the major dietary antioxidant nutrients: $\beta$-carotene, lycopene, catechin, vitamin C, and vitamin E (intake in $\mathrm{mg} / \mathrm{day}$ ); and the major pro-oxidant factors: heme iron (intake in $\mathrm{mg} /$ day), alcohol (intake in $\mathrm{g} / \mathrm{day}$ ), and smoking status (never, former, current) [21]. We also evaluated an antioxidant score (AOS) and oxidative balance score (OBS), which have been investigated in previous analyses [46, 47], including our study [21]. In our study, the AOS is the sum of quartile scores $(\mathrm{Q} 1=0 ; \mathrm{Q} 2=1 ; \mathrm{Q} 3=2 ; \mathrm{Q} 4=3$ ) of intake of $\beta$-carotene, lycopene, catechin, vitamin $\mathrm{C}$, and vitamin $\mathrm{E}$. The OBS additionally includes heme iron intake $(\mathrm{Q} 1=3 ; \mathrm{Q} 2=$ 2; $\mathrm{Q} 3=1 ; \mathrm{Q} 4=0$ ), alcohol consumption (abstainer $=3 ; 0.1-4 \mathrm{~g} /$ day $=2.25 ; 5-14 \mathrm{~g} /$ day $=1.5 ; 15-29 \mathrm{~g} / \mathrm{day}=0.75 ; \geq 30 \mathrm{~g} / \mathrm{day}=0$ ), and smoking status (never $=3$, former $=1.5$, current $=0$ ); these prooxidants are scored in the opposite way to the antioxidants.

The FFQ was validated and tested for reproducibility. Comparison with a 9-day dietary record showed that it was able to rank subjects adequately according to intake of the food groups and nutrients investigated [44]. The FFQ was also found to be a good indicator of nutrient intake over a period of at least 5 years [45]. Concentrations of specific nutrients in foods and beverages were derived from food composition tables based on data gathered in the Netherlands $[21,48]$. We excluded participants with incomplete or inconsistent dietary data, according to criteria described 
previously; 903 advanced PCa cases and 1,707 subcohort members had complete genotyping and dietary intake data (Figure 6.1) [44].

\section{Toenail selenium measurements}

Selenium concentrations were measured in toenail samples using instrumental neutron activation analysis of the ${ }^{77 \mathrm{~m}}$ Se isotope $[15,16]$. Each sample went through six cycles of 17-second irradiation at a thermal neutron flux of $3 \times 10^{16} \mathrm{~m}^{-2} \mathrm{~s}^{-1}$, 3-second decay, and 17 -second counting at $1 \mathrm{~cm}$ from a $40 \%$ germanium detector. The accuracy of the analysis was checked by a certified bovine liver standard (Standard Reference Material $1577 \mathrm{~b}$ of the US National Institute of Standards and Technology). We excluded participants who provided a toenail sample of less than $10 \mathrm{mg}$ because the selenium determination could otherwise be unreliable. A smaller version of the subcohort was used for the toenail selenium determination (70\%). The final analysis included 817 cases and 1,048 subcohort members who had complete toenail selenium data and genotyping data (Figure 6.1).

\section{Statistical analysis}

The risk of advanced (stage III/IV and IV) PCa in relation to oxidative stress-related genetic variants was assessed by age-adjusted Cox proportional hazards regression models. The SNPs were analyzed under a co-dominant and log-additive genetic model. The standard errors were estimated by using the robust Huber-White sandwich estimator to account for additional variance introduced by sampling from the cohort [49]. The proportional hazards assumption was tested using the scaled Schoenfeld residuals [50]. In none of the analyses the proportional hazards assumption was violated. The associations between the three GPX1 SNPs (rs17650792, rs1800668, and rs3448) and advanced PCa risk have been reported previously by our group [27], and are therefore not part of the present analysis.

We tested for multiplicative interactions between pro- and antioxidant exposures (quartiles or categories) and genotypes (dominant model) on advanced PCa (stage III/IV and IV) risk by using cross-product terms in the regression models and the Wald statistic. Exposure quartiles were based on the distribution of the variable in the subcohort. We used a dominant genetic model to increase study power, unless there was evidence of a recessive association in our study or previous studies. Interactions between GPX1 SNPs (rs17650792, rs1800668, and rs3448) and toenail selenium levels on advanced PCa risk have been reported previously by our group [27], and are therefore not part of the present analysis. The following factors were assessed as potential confounding variables: family history of PCa among first-degree relatives (yes/no), smoking status (never, former, or current), duration of smoking (years), frequency of smoking (cigarettes/day), non-occupational physical activity $(\leq 30$, 
$>30-60,>60-90,>90 \mathrm{~min} /$ day), body mass index $(<23,23-<25,25-<27,27-<30, \geq 30)$, height $(\mathrm{cm})$, diabetes (type I or II, yes/no), education level (primary school, lower vocational, high school, higher vocational/university), and intakes of energy (kcal/day) and calcium (g/day). These variables were identified as confounders when adding them, one at a time, to the age-adjusted model changed the point estimates by at least $10 \%$, which was the case for none of these variables.

To account for multiple statistical testing when identifying gene-environment interactions (total number of tests performed $=302$ ) we calculated false discovery rate (FDR) Q-values [51-53]. The FDR Q-value represents the expected proportion of falsepositive results when testing for significance. FDR Q-values are calculated by multiplying the P-value for interaction (calculated from each test from regression models) by the number of tests performed; and then dividing the multiplication by the rank order of each P-value (where rank order 1 is assigned to the smallest $P$ value) [53, 54]. We calculated an FDR Q-value for each pro- and antioxidant-specific interaction accounting for the total number of SNPs under investigation. We used an FDR Q-value threshold of 0.20 , which has been suggested for candidate gene studies [52, 53]. A more stringent threshold is often used in genome-wide association studies, which typically do not use prior (mechanistic) information to select candidate genes [55]. In this manuscript we presented SNP-environment interactions with a P for multiplicative interaction $<0.05$. For each statistical interaction we report the FDR Q-value and the hazard ratios (HRs) from the stratified analysis using a single reference category (lowest quartile or category of exposure and the common homozygote genotype).

All tests were two-sided with a P-value $<0.05$ considered to be statistically significant. Analyses were performed using STATA software (release 12, STATA Corporation, College Station, TX).

\section{Results}

Table 1 shows the baseline characteristics of advanced PCa cases and subcohort members. Compared with subcohort members, cases were more likely to have a firstdegree relative with PCa and less likely to have a history of diabetes.

Table 6.2 shows the associations between oxidative stress-related genetic variants and advanced PCa risk. CAT rs1001179 was associated with both stage III/IV and stage IV PCa risk, with HRs per minor A allele of $1.16(95 \% \mathrm{Cl}=1.01,1.33$; P for trend $=0.032)$ and $1.25(95 \% \mathrm{Cl}=1.07,1.46 ; \mathrm{P}$ for trend $=0.006)$, respectively. No other SNPs were associated with advanced PCa risk.

Table 6.3 shows the statistical interactions between oxidative stress-related genetic variants and pro- and antioxidant intake and status on advanced (stage III/IV and IV) PCa risk. We tested 151 SNP-environment interactions in relation to both stage 
III/IV and IV PCa risk and identified 15 statistically significant multiplicative interactions (stage III/IV PCa, $n=8$; stage IV PCa, $n=7$ ). Seven of these interactions met the FDR Qvalue $<0.20$ criterion; for stage III/IV PCa these involved intake of $\beta$-carotene (GPX1 rs17650792, hOGG1 rs1052133) and heme iron (GPX1 rs1800668 and rs3448), and for stage IV PCa these involved intake of catechin (SOD2 rs4880) and heme iron (hOGG1 rs1052133, SOD1 rs10432782). Stratified analyses using a single reference category (common homozygote genotype and lowest quartile of intake) showed: lowered risk for men with the AA genotype of GPX1 rs17650792 in the third quartile of $\beta$-carotene intake ( $H R=0.61 ; 95 \% \mathrm{Cl}: 0.40,0.93)$; elevated risk for men carrying the $\mathrm{G}$ allele of hOGG1 rs1052133 in the first $(\mathrm{HR}=1.49 ; 95 \% \mathrm{Cl}: 1.04,2.12)$ and third quartile of $\beta$ carotene intake $(H R=1.51 ; 95 \% \mathrm{Cl}: 1.08,2.11)$; lowered risk for men with the $A A$ genotype of GPX1 rs1800668 in the third quartile of heme iron intake (HR $=0.53 ; 95 \%$ $\mathrm{Cl}: 0.34,0.82)$; elevated risk for men carrying the G allele of hOGG1 rs1052133 in the first $(H R=1.48 ; 95 \% \mathrm{Cl}: 1.00,2.18)$ and second quartile of heme iron intake $(H R=1.66$; $95 \% \mathrm{Cl}: 1.12,2.46)$; and elevated risk for men carrying the $\mathrm{G}$ allele of SOD1 rs 10432782 in the first ( $\mathrm{HR}=2.14 ; 95 \% \mathrm{Cl}: 1.36,3.34)$ and second quartile of heme iron intake (HR $=1.61 ; 95 \% \mathrm{Cl}: 1.03,2.51)$. Of all 15 interactions, two displayed a decrease in risk over exposure categories. The first interaction was between SOD2 rs4880 and toenail selenium level on advanced PCa risk ( $P$ for interaction $=0.025 ; F D R Q=0.28$ ); compared with men with the common TT genotype in the lowest selenium quartile, carriers of the minor $\mathrm{C}$ allele (CT $+\mathrm{CC}$ ) in the highest selenium quartile had an HR of 0.44 (95\% Cl: 0.28, 0.69). The second interaction was between GPX1 rs17650792 and catechin intake on stage IV PCa risk (P for interaction $=0.042$; FDR $Q=0.29$ ); among men with the common homozygote AA genotype those in the highest versus lowest quartile had an HR of 0.38 (95\% Cl: $0.23,0.64)$. 


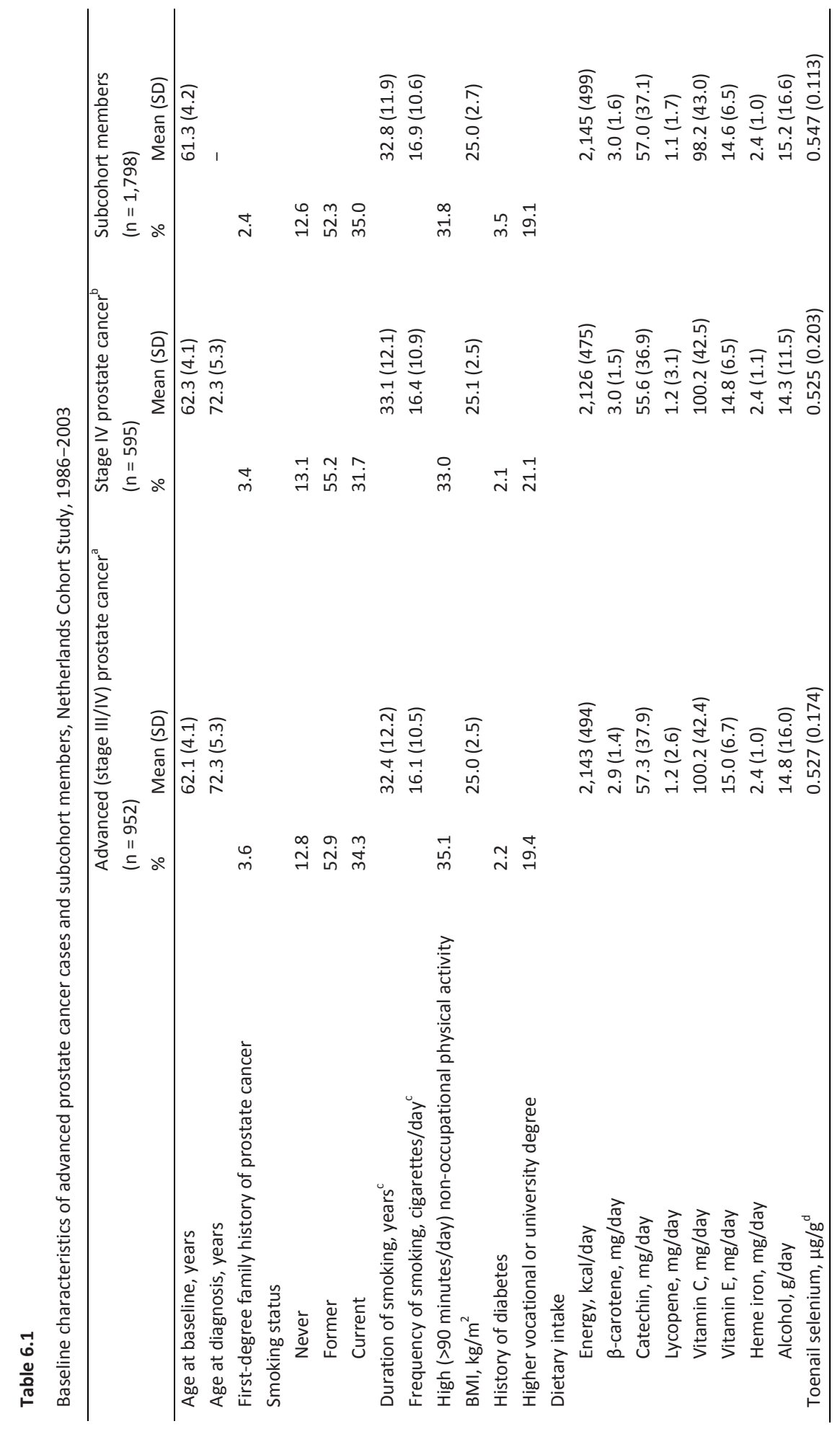

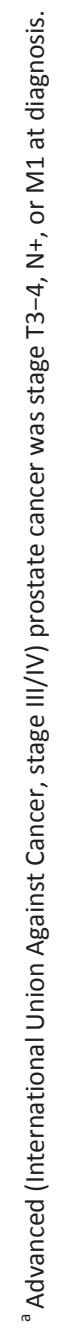




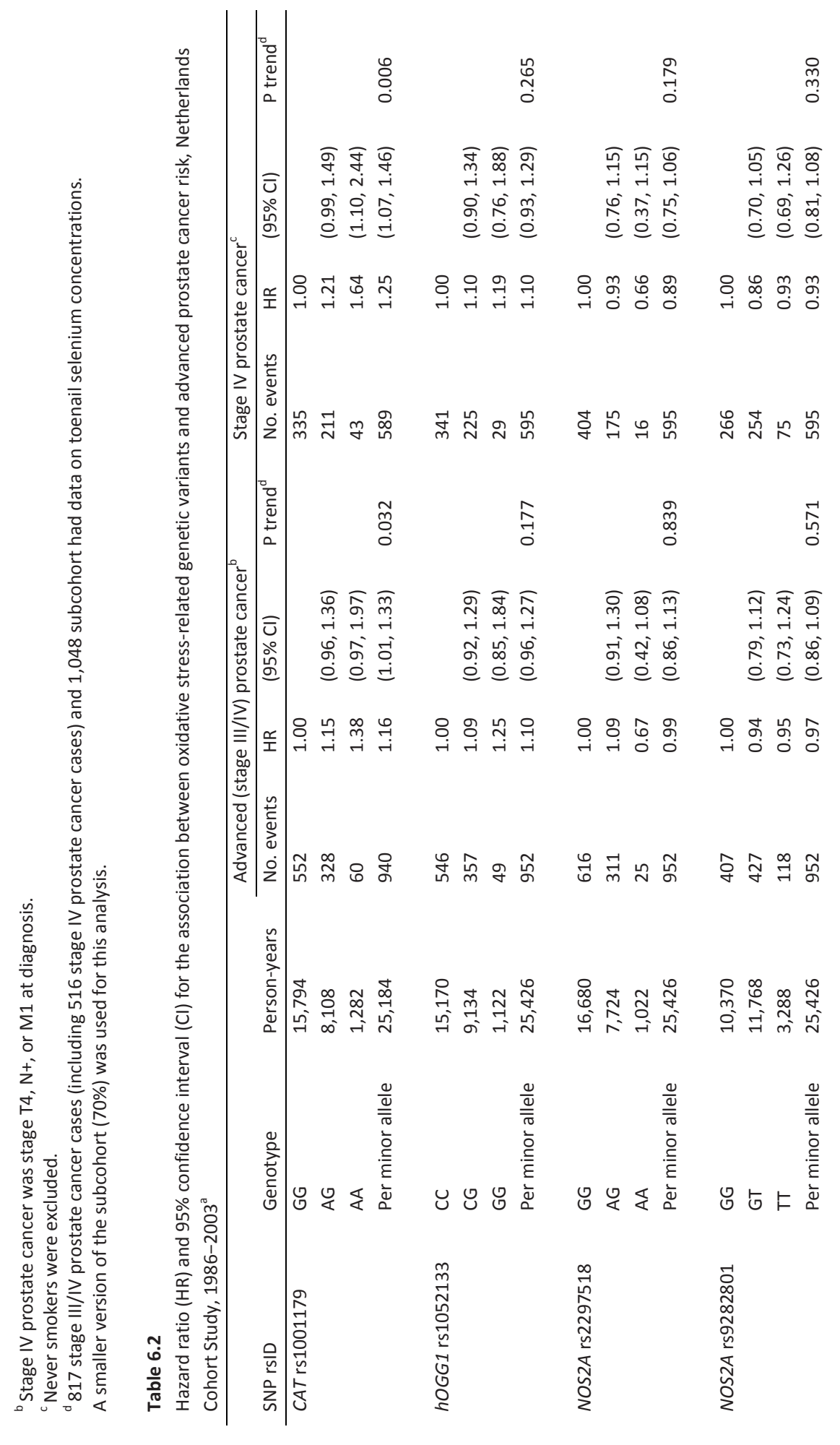




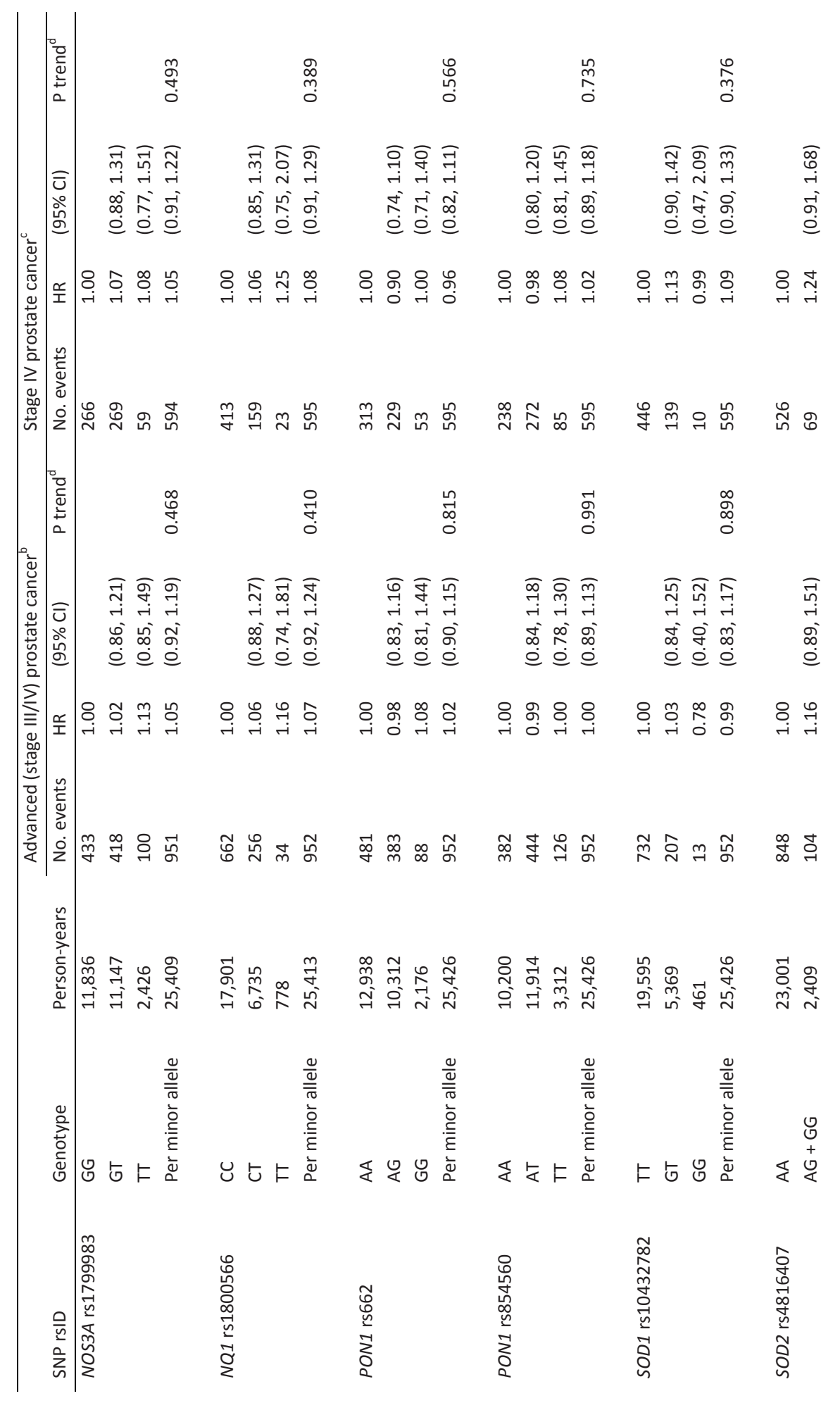




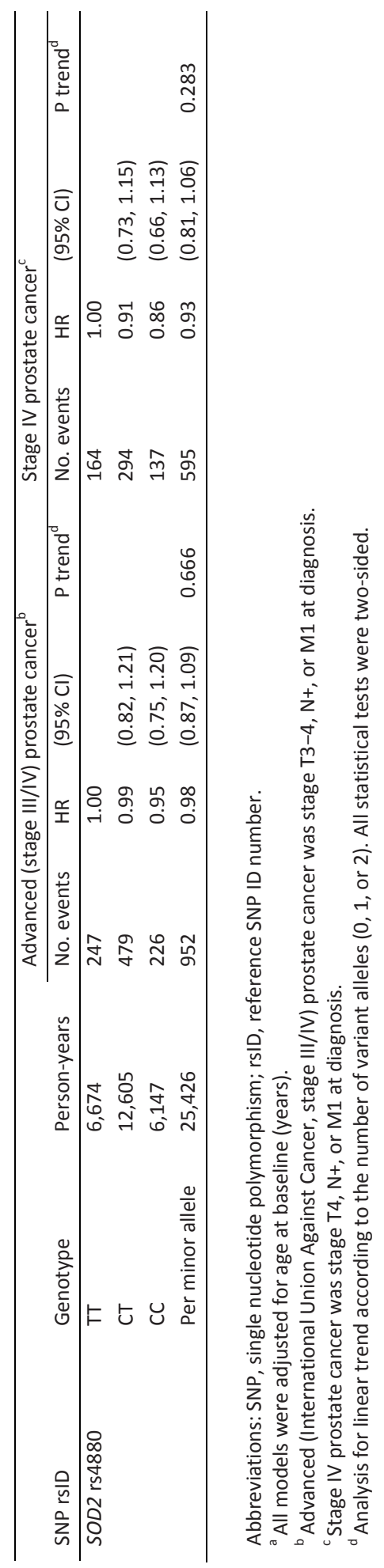




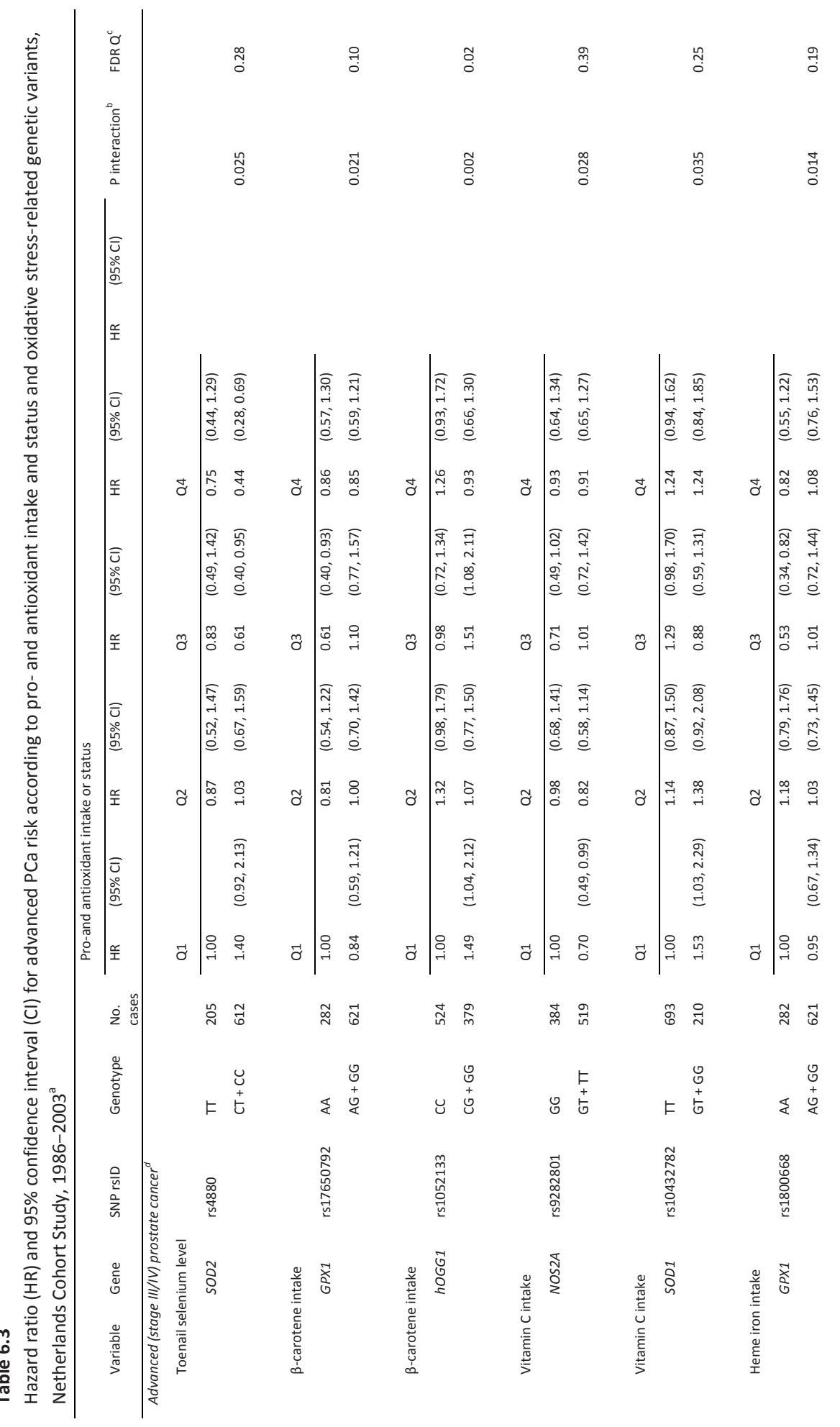




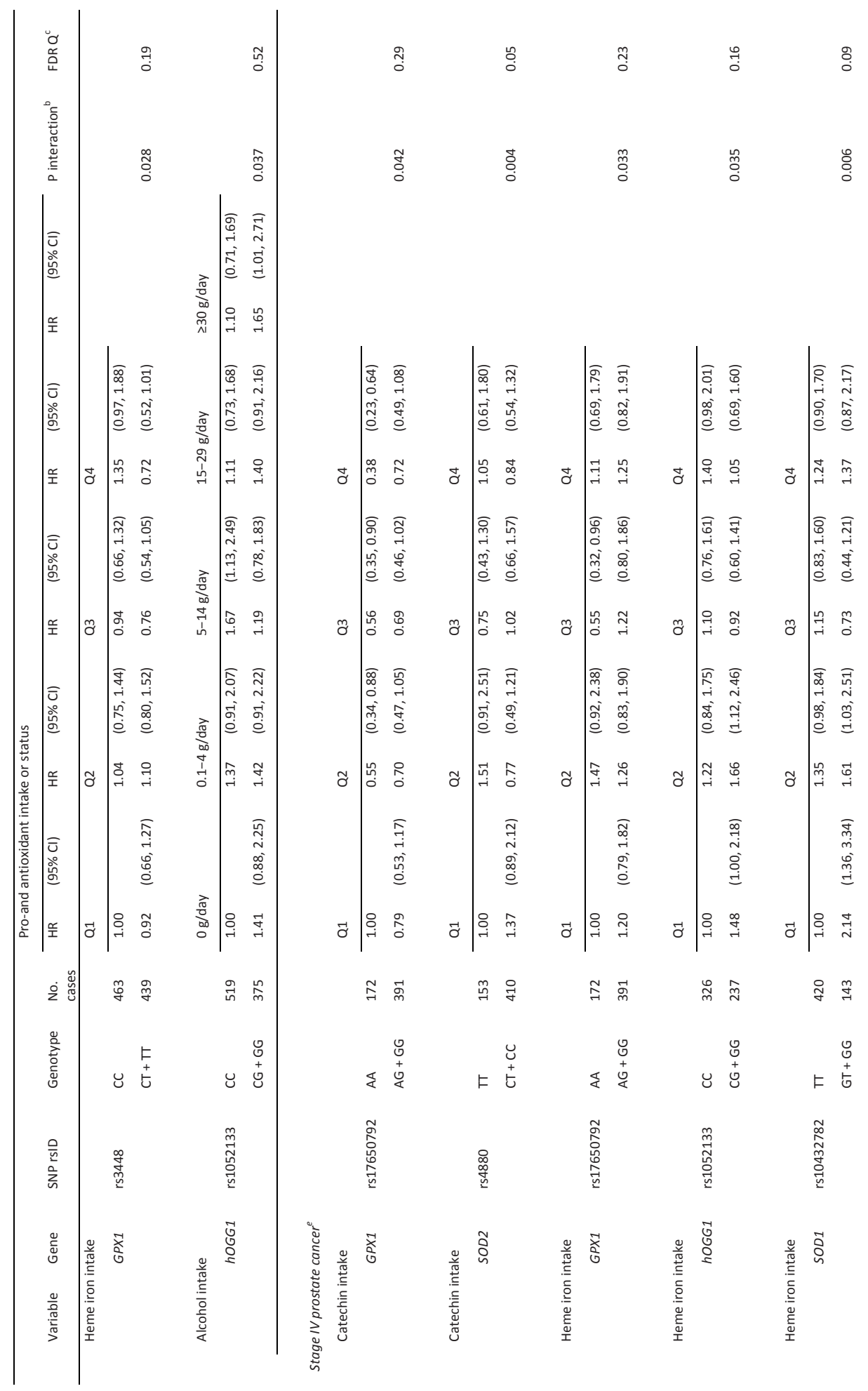




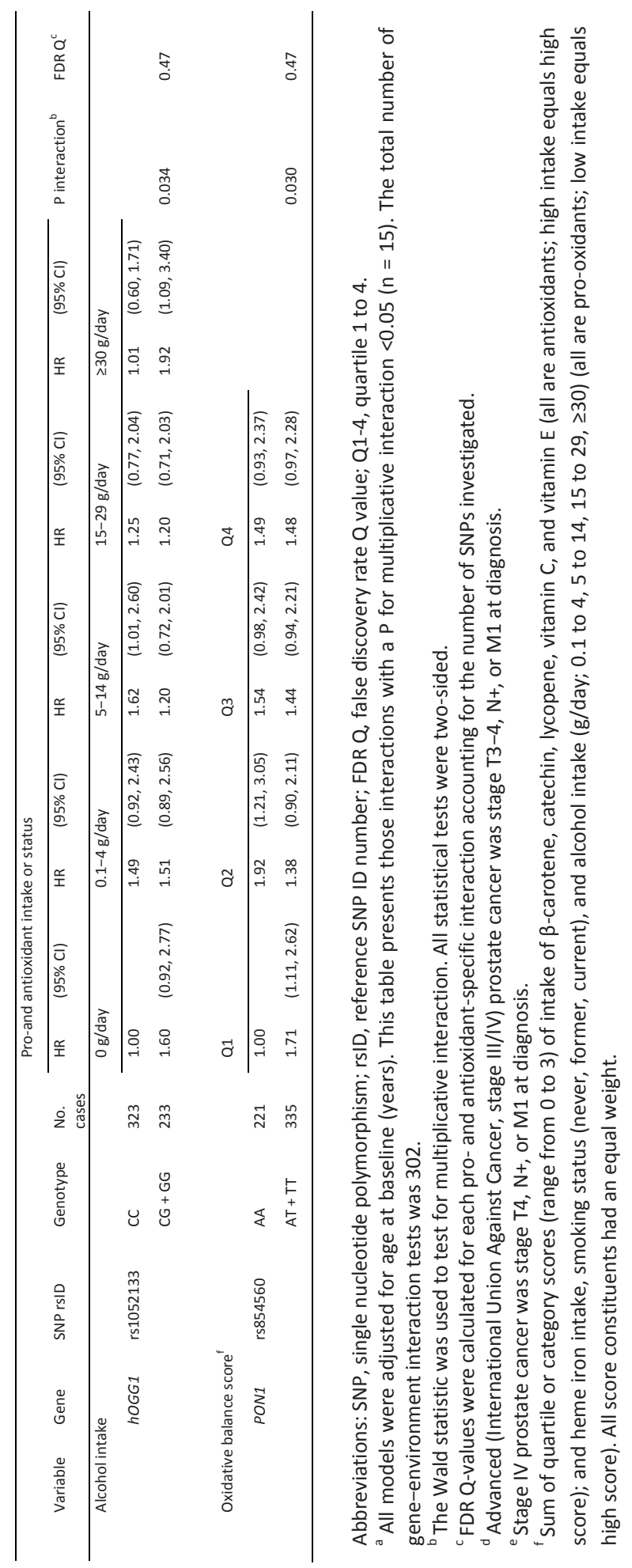




\section{Discussion}

In this large prospective study we evaluated oxidative stress-related genetic variants in relation to advanced $\mathrm{PCa}$ risk and examined potential interactions with pro- and antioxidant intake and status. The study showed an association between CAT rs1001179 and advanced PCa risk. Marginal associations were not observed for any of the other tested SNPs. Of the observed 15 statistically significant multiplicative gene-environment interactions, 7 retained significance after multiple comparison adjustment using an FDR Q-value threshold of 0.20. These interactions were on either stage III/IV or stage IV PCa risk and involved intake of $\beta$-carotene (GPX1, hOGG1), catechin (SOD2), and heme iron (GPX1 (two SNPS), hOGG1, SOD1).

Catalase is an endogenous antioxidant enzyme that neutralizes hydrogen peroxide, a type of ROS, by converting it to water and oxygen [5]. The enzyme plays an integral role in antioxidant defense against oxidative stress. CAT rs1001179 (A/G) (also known as $-262 \mathrm{C} / \mathrm{T}$ ) is a common polymorphism located in the promoter region of the gene [56]. A number of studies investigated associations between CAT rs1001179 and risk of cancer but mostly reported null findings [30]. Only few studies investigated this SNP in relation to PCa risk. In a nested case-control study (533 cases and 1,470 controls), Choi et al. showed no association between the SNP and overall PCa risk [40]. Recently, however, a small case-control study of PCa in Turkey (155 cases and 195 controls) showed that CAT rs1001179 was associated with overall PCa risk; men with the homozygote rare genotype compared to the homozygote common genotype had an $\mathrm{HR}$ of $1.57(95 \% \mathrm{Cl}=1.09,1.71)$ [57]. The authors also showed that among PCa cases the homozygote rare genotype was associated with advanced stage disease. Our analysis provides evidence of a role for CAT rs1001179 in PCa by showing that the rare allele of the SNP was associated with increased risk of advanced PCa. This finding is biologically plausible because the rare allele of CAT rs1001179 has been associated with lower catalase activity $[58,59]$. A reduced catalase activity may result in deficient antioxidant protection against oxidative stress, which could potentially translate into a higher risk of developing PCa. We found no evidence of a marginal association for the ten other SNPs investigated. This is striking because we selected candidate SNPs that have been associated with PCa risk in previous studies. The different outcome may result in part from the fact that we investigated advanced $\mathrm{PCa}$ and the prior studies mostly investigated overall PCa.

The significant gene-environment interactions (on stage III/IV or IV PCa) in our study involved intake of $\beta$-carotene (GPX1 rs17650792, hOGG1 rs1052133), catechin (SOD2 rs4880), and heme iron (GPX1 rs1800668 and rs3448, hOGG1 rs1052133, SOD1 rs10432782). These interactions, which have not been reported before, met the FDR $\mathrm{Q}$-value $<0.20$ criterion. The risk pattern of the interactions was unclear and could not 
be interpreted in terms of a dose-response relationship. We observed a significant altered risks for men in the third quartile of $\beta$-carotene and heme iron intake (GPX1, hOGG1) and evidence of a genotype effect (hOGG1, SOD1) for men with low heme iron intake. None of the statistical interactions showed a dose-response pattern that involved a distinct decrease or increase in risk over exposure categories. A number of epidemiological studies investigated associations of these nutrients (intake or levels) with PCa risk; and they generally do not support an association for $\beta$-carotene or heme iron [12, 20-22]. Although our group previously showed that higher catechin intake was associated with lower risk of advanced PCa [48], few other studies investigated the relationship and they generally do not support an association [60]. Given the limited evidence of an association between these dietary nutrients and (advanced) PCa risk, as well as that few other studies comprehensively evaluated oxidative stressrelated genetic variation in relation to pro- and antioxidant exposure and PCa risk; the positive results in this study require replication.

While the seven statistically significant interactions did not involve a clear dose-response pattern, two other multiplicative interactions ( $P$-value $<0.05$ ), that were however not significant after multiple testing correction (FDR Q-value $>0.20$ ), did involve a dose-response pattern. The risk pattern showed a decrease in risk over antioxidant exposure categories in one genotype subgroup but not the other. The first interaction was between selenium levels and SOD2 rs4880 on stage III/IV PCa risk and involved a 56\% lower risk for men carrying the $C$ allele in the highest quartile of selenium. This interaction has been reported previously in a nested case-control study in the Physicians' Health Study, showing a similar risk pattern as in our study [32]. The interaction is supported by mechanistic data and possibly results from an enzyme imbalance between SOD2 and GPX, which is selenium-dependent and removes hydrogen peroxide produced by SOD2 [61, 62]. The second interaction was between catechin intake and GPX1 rs17650792 on stage IV PCa risk and involved a 62\% lower risk for men with the homozygote AA genotype in the highest quartile of intake. As far as we know, there is no mechanistic data supporting this novel interaction.

Oxidative stress has been hypothesized to play an important role in PCa [9-11]. To shed additional light on this link we searched for gene-environment interactions between well-known oxidative stress-related gene variants and major pro- and antioxidant exposures. This type of hypothesis-driven pathway analysis is important to discover gene-environment interactions. Investigating interactions in gene-association studies with larger numbers of SNPs, including genome-wide association studies (GWAS), remains challenging because of the very large number of tests required. The most effective way to apply and detect interactions in the context of GWAS remains unresolved [63]. 
The most important strengths of our study include its prospective design, population-based approach, and long and nearly complete follow-up of the study population through linkage to cancer registries. We specifically evaluated advanced stage PCa because these cancers have a poor prognosis and are therefore clinically relevant [33]. Intake of selenium cannot be accurately estimated via an FFQ because of variations in the selenium content of soil and concomitant variability in the selenium content of foods [14]. We therefore used toenail selenium for exposure monitoring, which reflects long-term selenium intake [64].

Our study has some limitations. First, measurement of dietary intake using questionnaires may result in misclassification and potentially attenuated risk estimates, even though the study participants under study (aged 55 to 69 years) had relatively stable diets. Second, for most genes in our study we only selected 1 or 2 SNPs. The selected candidate SNPs were reported in prior studies to have a functional effect or an association with PCa risk and we may therefore have potentially missed important SNPs that modify advanced PCa risk.

In conclusion, this large prospective study on advanced PCa risk showed an association with a CAT polymorphism and identified seven novel statistically significant gene-environment interactions. Additional well-powered gene-association studies are needed to confirm these findings.

\section{References}

1. Droge W. Free radicals in the physiological control of cell function. Physiol Rev 2002;82(1):47-95.

2. Valko M, Rhodes CJ, Moncol J, et al. Free radicals, metals and antioxidants in oxidative stress-induced cancer. Chemico-biological interactions 2006;160(1):1-40.

3. Halliwell B. Reactive species and antioxidants. Redox biology is a fundamental theme of aerobic life. Plant Physiol 2006;141(2):312-322.

4. Fang YZ, Yang S, Wu G. Free radicals, antioxidants, and nutrition. Nutrition 2002;18(10):872-879.

5. Mates JM. Effects of antioxidant enzymes in the molecular control of reactive oxygen species toxicology. Toxicology 2000;153(1-3):83-104.

6. Finkel T, Holbrook NJ. Oxidants, oxidative stress and the biology of ageing. Nature 2000;408(6809):239247.

7. Muller FL, Lustgarten MS, Jang $Y$, et al. Trends in oxidative aging theories. Free Radical Biology and Medicine 2007;43(4):477-503.

8. Halliwell B. Oxidative stress and cancer: have we moved forward? The Biochemical journal 2007;401(1):1-11.

9. Bostwick DG, Burke HB, Djakiew D, et al. Human prostate cancer risk factors. Cancer 2004;101(10 Suppl):2371-2490.

10. Khandrika L, Kumar B, Koul S, et al. Oxidative stress in prostate cancer. Cancer letters 2009;282(2):125136.

11. Minelli A, Bellezza I, Conte C, et al. Oxidative stress-related aging: A role for prostate cancer? Biochimica et biophysica acta 2009;1795(2):83-91.

12. Vance TM, Su J, Fontham ET, et al. Dietary antioxidants and prostate cancer: a review. Nutrition and cancer 2013;65(6):793-801.

13. Wei MY, Giovannucci EL. Lycopene, Tomato Products, and Prostate Cancer Incidence: A Review and Reassessment in the PSA Screening Era. J Oncol 2012;2012:271063. 
14. Hurst R, Hooper L, Norat T, et al. Selenium and prostate cancer: systematic review and meta-analysis. The American journal of clinical nutrition 2012;96(1):111-122.

15. Geybels MS, Verhage BA, van Schooten FJ, et al. Advanced prostate cancer risk in relation to toenail selenium levels. Journal of the National Cancer Institute 2013;105(18):1394-1401.

16. van den Brandt PA, Zeegers MP, Bode $P$, et al. Toenail selenium levels and the subsequent risk of prostate cancer: a prospective cohort study. Cancer epidemiology, biomarkers \& prevention : a publication of the American Association for Cancer Research, cosponsored by the American Society of Preventive Oncology 2003;12(9):866-871.

17. Heinonen OP, Albanes D, Virtamo J, et al. Prostate cancer and supplementation with alpha-tocopherol and beta-carotene: incidence and mortality in a controlled trial. Journal of the National Cancer Institute 1998;90(6):440-446.

18. Duffield-Lillico AJ, Dalkin BL, Reid ME, et al. Selenium supplementation, baseline plasma selenium status and incidence of prostate cancer: an analysis of the complete treatment period of the Nutritional Prevention of Cancer Trial. BJU international 2003;91(7):608-612.

19. Klein EA, Thompson IM, Jr., Tangen CM, et al. Vitamin E and the risk of prostate cancer: the Selenium and Vitamin E Cancer Prevention Trial (SELECT). JAMA : the journal of the American Medical Association 2011;306(14):1549-1556.

20. Choi JY, Neuhouser ML, Barnett MJ, et al. Iron intake, oxidative stress-related genes (MnSOD and MPO) and prostate cancer risk in CARET cohort. Carcinogenesis 2008;29(5):964-970.

21. Geybels MS, Verhage BA, van Schooten FJ, et al. Measures of combined antioxidant and pro-oxidant exposures and risk of overall and advanced stage prostate cancer. Annals of epidemiology 2012;22(11):814-820.

22. Jakszyn PG, Allen NE, Lujan-Barroso L, et al. Nitrosamines and heme iron and risk of prostate cancer in the European prospective investigation into cancer and nutrition. Cancer epidemiology, biomarkers \& prevention : a publication of the American Association for Cancer Research, cosponsored by the American Society of Preventive Oncology 2012;21(3):547-551.

23. Rota M, Scotti L, Turati F, et al. Alcohol consumption and prostate cancer risk: a meta-analysis of the dose-risk relation. Eur J Cancer Prev 2012;21(4):350-359.

24. Zu K, Giovannucci E. Smoking and aggressive prostate cancer: a review of the epidemiologic evidence. Cancer causes \& control : CCC 2009;20(10):1799-1810.

25. Kang D, Lee KM, Park SK, et al. Functional variant of manganese superoxide dismutase (SOD2 V16A) polymorphism is associated with prostate cancer risk in the prostate, lung, colorectal, and ovarian cancer study. Cancer epidemiology, biomarkers \& prevention : a publication of the American Association for Cancer Research, cosponsored by the American Society of Preventive Oncology 2007;16(8):15811586.

26. Stevens VL, Rodriguez C, Talbot JT, et al. Paraoxonase 1 (PON1) polymorphisms and prostate cancer in the CPS-II Nutrition Cohort. The Prostate 2008;68(12):1336-1340.

27. Geybels MS, van den Brandt PA, Schouten $L$, et al. Selenoprotein gene variants, toenail selenium levels, and risk for advanced prostate cancer. Journal of the National Cancer Institute 2014;106(3):dju003.

28. Lee KM, Kang D, Park SK, et al. Nitric oxide synthase gene polymorphisms and prostate cancer risk. Carcinogenesis 2009;30(4):621-625.

29. Zhang J, Dhakal IB, Greene G, et al. Polymorphisms in hOGG1 and XRCC1 and risk of prostate cancer: effects modified by plasma antioxidants. Urology 2010;75(4):779-785.

30. Crawford A, Fassett RG, Geraghty DP, et al. Relationships between single nucleotide polymorphisms of antioxidant enzymes and disease. Gene 2012;501(2):89-103.

31. Eeles RA, Olama AA, Benlloch S, et al. Identification of 23 new prostate cancer susceptibility loci using the iCOGS custom genotyping array. Nature genetics 2013;45(4):385-391, 391e381-382.

32. Li H, Kantoff PW, Giovannucci E, et al. Manganese superoxide dismutase polymorphism, prediagnostic antioxidant status, and risk of clinical significant prostate cancer. Cancer research 2005;65(6):24982504.

33. Bostwick DG, Grignon DJ, Hammond ME, et al. Prognostic factors in prostate cancer. College of American Pathologists Consensus Statement 1999. Arch Pathol Lab Med 2000;124(7):995-1000.

34. van den Brandt PA, Goldbohm RA, van 't Veer P, et al. A large-scale prospective cohort study on diet and cancer in The Netherlands. Journal of clinical epidemiology 1990;43(3):285-295. 
35. van den Brandt PA, Schouten LJ, Goldbohm RA, et al. Development of a record linkage protocol for use in the Dutch Cancer Registry for epidemiological research. International journal of epidemiology 1990;19(3):553-558.

36. Goldbohm RA, van den Brandt PA, Dorant E. Estimation of the coverage of Dutch municipalities by cancer registries and PALGA based on hospital discharge data. Tijdschr Soc Gezondheidsz. 1994;19(3):80-84.

37. Barlow WE, Ichikawa L, Rosner D, et al. Analysis of case-cohort designs. Journal of clinical epidemiology 1999;52(12):1165-1172.

38. Sobin L, Gospodarowicz M, Wittekind C, et al. TNM Classification of Malignant Tumors. 7th ed. Oxford, UK: Wiley-Blackwell; 2009.

39. van Breda SG, Hogervorst JG, Schouten L, et al. Toenails: an easily accessible and long-term stable source of DNA for genetic analyses in large-scale epidemiological studies. Clinical chemistry 2007;53(6):1168-1170.

40. Choi JY, Neuhouser ML, Barnett M, et al. Polymorphisms in oxidative stress-related genes are not associated with prostate cancer risk in heavy smokers. Cancer epidemiology, biomarkers \& prevention : a publication of the American Association for Cancer Research, cosponsored by the American Society of Preventive Oncology 2007;16(6):1115-1120.

41. Geybels MS, Hutter CM, Kwon EM, et al. Variation in selenoenzyme genes and prostate cancer risk and survival. The Prostate 2013;73(7):734-742.

42. Steinbrecher A, Rohrmann S, Timofeeva $M$, et al. Dietary glucosinolate intake, polymorphisms in selected biotransformation enzymes, and risk of prostate cancer. Cancer epidemiology, biomarkers \& prevention : a publication of the American Association for Cancer Research, cosponsored by the American Society of Preventive Oncology 2010;19(1):135-143.

43. Abe $M$, Xie W, Regan MM, et al. Single-nucleotide polymorphisms within the antioxidant defence system and associations with aggressive prostate cancer. BJU international 2011;107(1):126-134.

44. Goldbohm RA, van den Brandt PA, Brants HA, et al. Validation of a dietary questionnaire used in a largescale prospective cohort study on diet and cancer. European journal of clinical nutrition 1994;48(4):253265.

45. Goldbohm RA, van 't Veer P, van den Brandt PA, et al. Reproducibility of a food frequency questionnaire and stability of dietary habits determined from five annually repeated measurements. European journal of clinical nutrition 1995;49(6):420-429.

46. Lakkur S, Goodman M, Bostick RM, et al. Oxidative balance score and risk for incident prostate cancer in a prospective U.S. cohort study. Annals of epidemiology 2014.

47. Agalliu I, Kirsh VA, Kreiger N, et al. Oxidative balance score and risk of prostate cancer: results from a case-cohort study. Cancer Epidemiol 2011;35(4):353-361.

48. Geybels MS, Verhage BA, Arts IC, et al. Dietary flavonoid intake, black tea consumption, and risk of overall and advanced stage prostate cancer. American journal of epidemiology 2013;177(12):1388-1398.

49. Lin DY, Wei LY. The robust inference for the Cox proportional hazards model. J Am Stat Assoc. 1989;84(408):1074-1078.

50. Grambsch PM, Therneau TM. Proportional hazards tests and diagnostics based on weighted residuals. Biometrika 1994;81(3):515-526.

51. Benjamini Y, Hochberg Y. Controlling the False Discovery Rate: a Practical and Powerful Approach to Multiple Testing. J. R. Statist. Soc. 1995;57(1):289-300.

52. Karami S, Andreotti G, Koutros S, et al. Pesticide Exposure and Inherited Variants in Vitamin D Pathway Genes in Relation to Prostate Cancer. Cancer epidemiology, biomarkers \& prevention : a publication of the American Association for Cancer Research, cosponsored by the American Society of Preventive Oncology 2013.

53. Smith NL, Hindorff LA, Heckbert SR, et al. Association of genetic variations with nonfatal venous thrombosis in postmenopausal women. Jama-Journal of the American Medical Association 2007;297(5):489-498.

54. Storey JD, Tibshirani R. Statistical significance for genomewide studies. Proceedings of the National Academy of Sciences of the United States of America 2003;100(16):9440-9445.

55. Benjamini Y, Yekutieli D. Quantitative trait Loci analysis using the false discovery rate. Genetics 2005;171(2):783-790.

56. Forsberg L, Lyrenas L, de Faire U, et al. A common functional C-T substitution polymorphism in the promoter region of the human catalase gene influences transcription factor binding, reporter gene 
transcription and is correlated to blood catalase levels. Free radical biology \& medicine 2001;30(5):500505.

57. Tefik T, Kucukgergin C, Sanli O, et al. Manganese superoxide dismutase Ile58Thr, catalase C-262T and myeloperoxidase G-463A gene polymorphisms in patients with prostate cancer: relation to advanced and metastatic disease. BJU international 2013;112(4):E406-414.

58. Ahn J, Gammon MD, Santella RM, et al. Associations between breast cancer risk and the catalase genotype, fruit and vegetable consumption, and supplement use. American journal of epidemiology 2005;162(10):943-952.

59. Ahn J, Nowell S, McCann SE, et al. Associations between catalase phenotype and genotype: modification by epidemiologic factors. Cancer epidemiology, biomarkers \& prevention : a publication of the American Association for Cancer Research, cosponsored by the American Society of Preventive Oncology 2006;15(6):1217-1222.

60. Wang Y, Stevens VL, Shah R, et al. Dietary flavonoid and proanthocyanidin intakes and prostate cancer risk in a prospective cohort of US men. American journal of epidemiology 2014;179(8):974-986.

61. Li H, Kantoff PW, Giovannucci E, et al. Manganese superoxide dismutase polymorphism, prediagnostic antioxidant status, and risk of clinical significant prostate cancer. Cancer research 2005;65(6):24982504.

62. Sutton A, Khoury H, Prip-Buus C, et al. The Ala16Val genetic dimorphism modulates the import of human manganese superoxide dismutase into rat liver mitochondria. Pharmacogenetics 2003;13(3):145-157.

63. Cornelis MC, Tchetgen EJ, Liang L, et al. Gene-environment interactions in genome-wide association studies: a comparative study of tests applied to empirical studies of type 2 diabetes. American journal of epidemiology 2012;175(3):191-202.

64. Slotnick MJ, Nriagu JO. Validity of human nails as a biomarker of arsenic and selenium exposure: $A$ review. Environmental research 2006;102(1):125-139. 

Chapter 7: Discussion 


\subsection{Main study findings}

First, we investigated associations between selenium levels measured in toenail samples and advanced PCa risk and showed that men with the highest selenium levels had a more than $60 \%$ lower risk compared to men with the lowest levels (Chapter 2; Table 7.1). Second, we studied common genetic variation in the major selenoprotein genes SEPP1 (5 tagging SNPs) and GPX1 (3 tagging SNPs) in relation to advanced PCa risk and examined potential interactions with toenail selenium levels (Chapter 3; Table 7.2). The analysis showed that one SEPP1 polymorphism and two GPX1 polymorphisms were associated with advanced PCa risk. Toenail selenium levels were inversely associated with advanced PCa risk independently of common genetic variation in SEPP1 and GPX1. Third, we studied intake of other antioxidant nutrients and prooxidant exposures (individually and using combined scores) in relation to PCa risk (Chapters 4, 5). Results of these analyses are summarized in Table 7.1. While most exposure variables were not associated, intake of specific flavonoids (i.e., catechin, epicatechin, myricetin, and kaempferol) and consumption of black tea (a major source of flavonoids) were associated with a modest decrease in risk of advanced PCa. Finally, we studied candidate oxidative stress-related genetic variants in relation to advanced PCa risk and examined potential interactions with pro- and antioxidant exposures (No. of gene variants $=14$; No. of exposure variables $=11$ ) (Chapter 6$)$. The results of these analyses are summarized in Table 7.2. We found that a CAT polymorphism was associated with advanced PCa risk and identified seven statistically significant geneenvironment interactions ( $P$-value $<0.05$ and FDR Q-value $<0.20$ ), which involved intake of $\beta$-carotene (GPX1, hOGG1), catechin (SOD2), and heme iron (GPX1 (2 SNPs), hOGG1, SOD2). 
Table 7.1

Pro- and antioxidant dietary and lifestyle factors in relation to overall and advanced prostate cancer risk, Netherlands Cohort Study, 1986-2003

\begin{tabular}{|c|c|c|}
\hline Factor & Overall prostate cancer & Advanced prostate cancer ${ }^{a}$ \\
\hline Toenail selenium level & $\downarrow^{\mathrm{b}}$ & $\downarrow$ \\
\hline \multicolumn{3}{|l|}{ Dietary intake of } \\
\hline$\beta$-carotene & - & - \\
\hline Total catechin & - & $\downarrow$ \\
\hline Catechin & - & - \\
\hline Epicatechin & - & $\downarrow$ \\
\hline Total flavonol & - & - \\
\hline Quercetin & - & - \\
\hline Kaempferol & - & $\downarrow$ \\
\hline Myricetin & - & $\downarrow$ \\
\hline Lycopene & - & - \\
\hline Vitamin C & - & - \\
\hline Vitamin E & - & - \\
\hline Heme iron & - & - \\
\hline Smoking Status & - & - \\
\hline Alcohol consumption & - & - \\
\hline Antioxidant Score & - & - \\
\hline Oxidative Balance Score & - & - \\
\hline Black tea consumption & - & $\downarrow$ \\
\hline
\end{tabular}

Symbols: $\downarrow$, inverse association; -, no association.

${ }^{a}$ We used two definitions of advanced PCa: stage III/IV PCa (T3-4, N+, or M1 at diagnosis) and stage IV PCa (T4, $\mathrm{N}+$, or $\mathrm{M} 1$ at diagnosis).

${ }^{b}$ The inverse association between toenail selenium levels and overall PCa risk in the Netherlands Cohort Study was not part of the present thesis but has been reported previously [1]. This analysis included 6.3 years of follow-up. All other results presented in this table were based on 17.3 years of follow-up. 
Table 7.2

Associations between genetic variants in selenoprotein and oxidative stress-related genes and advanced prostate cancer risk, and interactions with pro- and antioxidant exposures, Netherlands Cohort Study, $1986-2003^{\mathrm{a}}$

\begin{tabular}{|c|c|c|c|}
\hline Gene $^{b}$ & SNP rsID & $\begin{array}{l}\text { Associated with advanced } \\
\text { PCa (risk allele) }\end{array}$ & $\begin{array}{l}\text { Gene-environment interaction } \\
\text { (environmental factor) }^{d}\end{array}$ \\
\hline \multirow[t]{5}{*}{ SEPP1 } & rs13168440 & - & - \\
\hline & rs230813 & - & - \\
\hline & rs230819 & - & - \\
\hline & rs3877899 & - & - \\
\hline & rs7579 & Major G & - \\
\hline \multirow[t]{3}{*}{ GPX1 } & rs17650792 & Minor G & $\beta$-carotene; catechin, heme iron \\
\hline & rs1800668 & Major C & Heme iron \\
\hline & rs3448 & - & Heme iron \\
\hline$C A T$ & rs1001179 & Minor A & - \\
\hline hOGG1 & rs1052133 & - & $\boldsymbol{\beta}$-carotene; alcohol ${ }^{\mathrm{e}}$; heme iron \\
\hline \multirow[t]{2}{*}{ NOS2A } & rs2297518 & - & - \\
\hline & rs9282801 & - & Vitamin C \\
\hline NOS3A & rs1799983 & - & - \\
\hline NQ1 & rs1800566 & - & - \\
\hline \multirow[t]{2}{*}{ PON1 } & rs662 & - & - \\
\hline & rs854560 & - & Oxidative balance score \\
\hline SOD1 & rs10432782 & - & Vitamin C; heme iron \\
\hline \multirow[t]{2}{*}{ SOD2 } & rs4816407 & - & - \\
\hline & rs4880 & - & Selenium; catechin \\
\hline
\end{tabular}

Symbols: -, no association or gene-environment interaction.

${ }^{a}$ We used two definitions of advanced PCa: stage III/IV PCa (T3-4, N+, or M1 at diagnosis) and stage IV PCa (T4, N+, or M1 at diagnosis).

${ }^{\mathrm{b}}$ SEPP1 and GPX1 SNPs were selected as tagging SNPs $\left(r^{2} \geq 0.8\right.$; minor allele frequency $\left.\geq 10 \%\right)$. All other SNPS were candidate SNPs reported in prior studies to have a functional effect or an association with PCa risk (and minor allele frequency $\geq 10 \%$ ).

c When marginally associated (using a co-dominant or log-additive model) we report the risk allele associated with an elevated risk.

${ }^{\mathrm{d}}$ We report multiplicative gene-environment interactions with a $\mathrm{P}$ for interaction $<0.05$ (Wald test). The environmental factor is highlighted in bold in case the interaction remained statistically significant after multiple testing adjustment using FDR and an FDR Q-value threshold of 0.20. The following pro- and antioxidant environmental factors were examined: toenail selenium levels, intake of $\beta$-carotene, lycopene, catechin, vitamin $\mathrm{C}$, vitamin $\mathrm{E}$, and heme iron, alcohol consumption, smoking status, antioxidant score, and 
oxidative balance score. For SEPP1 SNPs, we only investigated potential interactions with selenium and not the other exposures.

${ }^{\mathrm{e}}$ The interaction between alcohol consumption and hOGG1 rs1052133 was significant for both stage III/IV and stage IV PCa risk $(\mathrm{P}<0.05)$.

\subsection{Interpretation of study findings}

7.2.1 Selenium and prostate cancer risk - Is low selenium status a risk factor for prostate cancer?

Many prospective analyses, including our study, showed that selenium levels are inversely associated with PCa risk [1, 2]. The two clinical trials on selenium supplementation and PCa risk had mixed findings: the Nutritional Prevention of Cancer (NPC) trial showed, in a secondary analysis, that selenium supplementation reduced PCa risk [3], but the Selenium and Vitamin E Cancer Prevention Trial (SELECT), which was primarily designed to study PCa, found no beneficial effect of selenium on PCa risk [4].

The Nutritional Prevention of Cancer trial, Selenium and Vitamin E Cancer Prevention Trial, and Netherlands Cohort Study - Adequate selenium status for the prevention of prostate cancer?

A major difference between the two clinical trials on selenium and PCa risk, the NPC trial and SELECT, is the baseline selenium level (Figure 7.1). The NPC trial, which showed a preventive effect of selenium supplementation on PCa risk, was conducted among subjects from the southeastern US [3]. This region was selected because it has relatively low soil selenium concentrations. A consequence of these lower selenium soil concentrations is that individuals from these regions have a lower selenium intake and status. The mean baseline plasma selenium level of men in the NPC trial was 115 $\mu \mathrm{g} / \mathrm{L}$ (standard deviation (SD) $=22 \mu \mathrm{g} / \mathrm{L}$ ) [5]. SELECT, which showed no preventive effect of selenium supplementation on PCa risk, was conducted among men from various regions in the US, Canada, and Puerto Rico [6]. These individuals have relatively high selenium levels. The median baseline plasma selenium level in SELECT was $136 \mu \mathrm{g} / \mathrm{L}$ (interquartile range = 122-152). Because SELECT, unlike the NPC trial, was conducted among American men with higher selenium levels, average baseline selenium in SELECT may have already been sufficiently high, explaining why additional selenium supplementation had no effect on PCa risk. That baseline selenium in SELECT may have been adequate is supported by additional results from the NPC trial showing that the preventive effect of selenium on PCa risk was restricted to men in the lowest two tertiles of baseline selenium, i.e. lower than $123 \mu \mathrm{g} / \mathrm{L}$ plasma selenium (Figure 7.1) [5]. Most men in SELECT had a baseline selenium level higher than this cut point. 


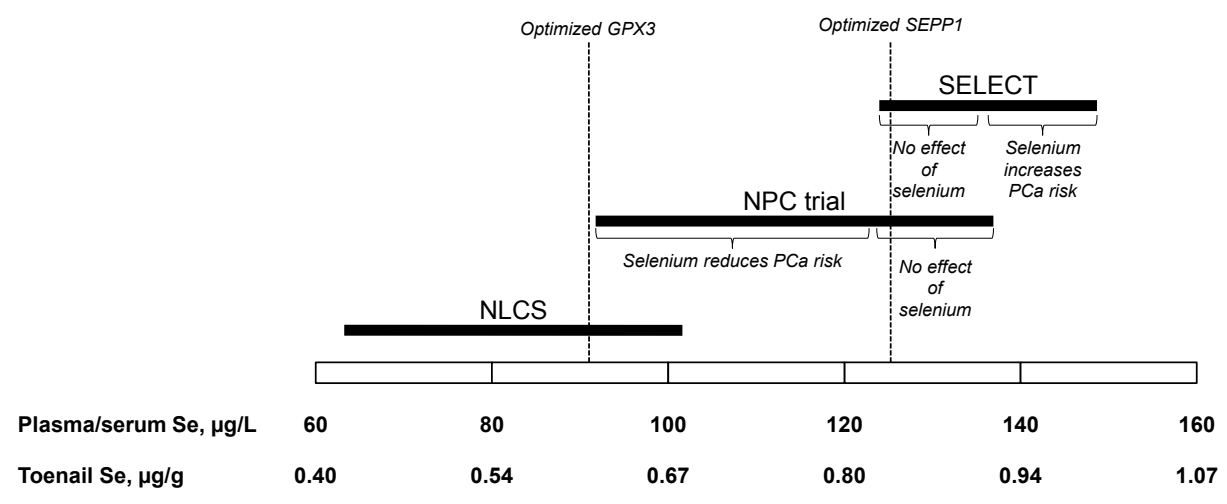

\section{Figure 7.1}

Range of (baseline) selenium levels in the Netherlands Cohort Study (NLCS), Nutritional Prevention of Cancer (NPC) trial, and Selenium and Vitamin E Cancer Prevention Trial (SELECT), and approximate selenium levels required to optimize the plasma selenoproteins (GPX3 and SEPP1)

The selenium range (black bar) in the NLCS is the average toenail selenium level of male subcohort members \pm standard deviation (Chapter 2 of this thesis). To convert toenail selenium levels to plasma selenium levels we used the simple conversion ratio described by Waters et al.: plasma selenium $(\mu \mathrm{g} / \mathrm{L})=$ toenail selenium $(\mu \mathrm{g} / \mathrm{g}) / 0.0067$ [15]. The selenium range in the NPC trial is the average baseline plasma selenium level \pm standard deviation [4]. The selenium range in SELECT is the interquartile range of baseline plasma selenium levels [5]. Plasma selenium levels required to optimize activity or concentration of the plasma selenoproteins GPX3 ( $\pm 92 \mu \mathrm{g} / \mathrm{L}$ ) and SEPP1 ( $\pm 125 \mu \mathrm{g} / \mathrm{L}$ ) are marked by dotted vertical lines [8-10]. In the NPC trial, selenium supplementation was associated with reduced PCa risk [2, 92], but when stratified by baseline plasma selenium the inverse association was only observed for men in the first two tertiles of baseline selenium (<123 $\mu \mathrm{g} / \mathrm{L})$ [4]. In SELECT, selenium supplementation was not associated with PCa risk overall, but when stratified by baseline toenail selenium men with the highest levels (>0.90 $\mu \mathrm{g} / \mathrm{g}) \mathrm{had}$ an increased risk of high-grade PCa from selenium supplementation [11].

Adequate selenium status is possibly related to plasma selenium levels needed to optimize activity or concentration of the plasma selenoproteins. GPX3 and SEPP1 are the plasma selenoproteins [7]. Both proteins serve as selenium nutritional markers and plasma GPX3 activity has been used to set dietary reference values for selenium [8]. The plasma selenium concentration needed to maximize GPX3 activity is approximately $92 \mu \mathrm{g} / \mathrm{L}[9,10]$, which is expected to be sufficient to optimize activity of the other isoforms of GPX [10]. SEPP1 has a maximum concentration at a higher plasma selenium concentration of approximately $125 \mu \mathrm{g} / \mathrm{L}[10,11]$. Baseline plasma selenium levels in SELECT were clearly higher than the selenium concentrations needed to optimize these selenoproteins (Figure 7.1). This again suggests that most SELECT participants may already have had an adequate selenium intake at baseline. Furthermore, mean post-treatment plasma selenium in SELECT was $225 \mu \mathrm{g} / \mathrm{L}$ [12], 
which is almost two times the concentration needed to optimize the plasma selenoproteins.

Selenium concentrations in our study among men from the Netherlands were much lower than those in SELECT and the NPC trial (Figure 7.1). This is a result of the typically lower selenium soil concentrations in Europe $[8,13,14]$. In our study, we used toenail selenium concentrations for exposure monitoring. Toenail selenium status is an excellent selenium status biomarker because it reflects long-term selenium intake [2, 15]. To convert toenail selenium levels to plasma selenium levels we used the simple conversion ratio described by Waters et al.: plasma selenium $(\mu \mathrm{g} / \mathrm{L})=$ toenail selenium $(\mu \mathrm{g} / \mathrm{g}) / 0.0067$ [16]. In our study, the average toenail selenium level of 0.55 (SD $=0.13$ ) approximates a plasma selenium level of $0.82(S D=0.19)$. Figure 7.1 shows that while the range of selenium in our study falls below the expected selenium level needed to optimize SEPP1, men with the highest selenium levels in our study are expected to have optimal GPX3 activity. Selenium status of most men in our study was also lower than $123 \mu \mathrm{g} / \mathrm{L}$ plasma selenium; men in the NPC trial with a baseline plasma selenium level higher than $123 \mu \mathrm{g} / \mathrm{L}$ had no effect of selenium supplementation on PCa risk [5]. Based on this prior scientific evidence, selenium levels in our study are clearly in the range were additional selenium intake is expected to be beneficial.

That baseline selenium status may be important for the efficacy of selenium supplementation in PCa prevention is further highlighted by an additional investigation in SELECT [12]. Kristal et al. recently analyzed SELECT data stratified by baseline toenail selenium levels. The study showed that while selenium supplementation had no effect among men with low baseline selenium status (<60th percentile of toenail selenium) it increased risk of high-grade PCa (Gleason 7-10) among men with higher selenium status. Baseline toenail selenium levels in SELECT (mean $=0.89 \mu \mathrm{g} / \mathrm{g}$ ) were much higher than toenail selenium levels in the Netherlands Cohort Study (mean $=0.55$ $\mu \mathrm{g} / \mathrm{g})$. The cut point for the highest selenium quintile in our study was $0.617 \mu \mathrm{g} / \mathrm{g}$; only $0.9 \%$ of PCa cases in SELECT had baseline selenium levels below this threshold. SELECT data demonstrate that high-dose selenium supplementation $(200 \mathrm{mg}$ selenomethionine daily) does not reduce PCa risk among men who are in the range of selenium status that is common in US men and may even increase risk among men at the highest range of selenium intake. These data do not rule out that selenium supplementation may benefit men who are not selenium-replete but have lower selenium levels comparable to those found in many European countries. A possible explanation for the unexpected finding in SELECT comes from a feeding trial of selenium supplementation in dogs, the only non-human animal model of spontaneous PCa [16]. This study showed a non-linear U-shaped dose-response relationship between selenium status and the extent of DNA damage in the aging prostate. That 
dietary nutrients may have a U-shaped relationship with human disease/mortality has been reported for some nutrients other than selenium [17].

\section{Blood and toenail selenium and novel selenium status biomarkers}

Much of our understanding of the complex dose-response relationship between selenium and PCa risk comes from studies using blood (plasma/serum) and toenail selenium concentrations as selenium status biomarkers. Blood and toenail selenium are valid selenium status biomarkers that respond over a wide range of selenium intake [8]. Both markers, however, may not accurately reflect selenium status in all situations. Levels of selenium in blood and toenails may depend on the type of selenium species in foods and supplements, subject characteristics, and dietary and other lifestyle exposures $[8,15]$. Several novel selenium status biomarkers are being examined including selenoprotein gene expression levels in blood [8]. Future studies on selenium and PCa risk should consider using other selenium status biomarkers, in addition to blood and toenail selenium, which may provide important insights in the dose-response relationship between selenium status and PCa risk.

\subsubsection{Selenium and prostate cancer risk - A role for selenoprotein genes?}

The biological activities of selenium mainly depend on selenoproteins [14, 18]. Genetic variation in the genes encoding selenoproteins may influence PCa risk directly or by modifying the effect of selenium (gene-environment interaction). There have been a few prior studies on selenoprotein genes and PCa risk. The studies showed significant associations with PCa risk and survival for variants in a number of selenoprotein genes including SEPP1 [19, 20], GPX1 [21, 22], SEP15 [23], and TXNR1-2 [24]. The associations have only been reported in one or two studies and therefore require confirmation from other studies. Genome-wide association studies of PCa have not yet identified risk variants in selenoprotein genes [25].

In our study we investigated common genetic variation in SEPP1 and GPX1 (using tagging SNPS) in relation to toenail selenium levels and advanced PCa risk. We identified both genes as important candidates to modify PCa risk and the selenium-PCa association because (1) polymorphisms in both genes have previously been associated with PCa risk [14, 19, 22], and (2) both genes encode proteins with essential biological functions. SEPP1 is the main selenium transport protein in plasma and delivers selenium from the liver to other tissues [7]. GPX1 was the first selenoprotein identified and its strong antioxidant potential has been demonstrated in many mechanistic studies [26]. Furthermore, both genes come to expression in the prostate and there is some evidence that both genes are down-regulated in PCa [2628]. 
The results of our study showed that common genetic variants in SEPP1 (rs7579) and GPX1 (rs1800668, rs17650792) were associated with advanced PCa risk. We showed that the A allele of SEPP1 rs7579 was associated with decreased risk, which has not been reported before. The association was with stage IV PCa risk only. The association is supported by a study showing that men carrying this allele have higher serum concentrations of SEPP1 [29]. This polymorphism, its potential biological consequence, and the potential association it may have with PCa risk require further study. The GPX1 SNPs rs1800668 and rs17650792 are poorly studied genetic variants that have not been associated with PCa risk before. GPX1 rs17650792 is located near the GPX1 loci and is not correlated with other common genetic variants in GPX1. GPX1 rs 1800668 is located in the promoter of GPX1 and is in high linkage disequilibrium (LD) with GPX1 rs1050450 ( $\left.r^{2}=0.9\right)$. GPX1 rs1050450 resides in the coding region and this SNP may have functional consequences because it results in an amino acid substitution of proline with leucine [30, 31]. The observed association of GPX1 rs1800668 in our study may be due to its high correlation with GPX1 rs1050450, which may be the true functional polymorphism. This is supported by an earlier small case-control study, which showed that the variant allele of GPX1 rs1050450 was associated with reduced PCa risk [21]. In our study we observed no evidence that common SEPP1 and GPX1 variants modify the inverse association between toenail selenium levels and advanced PCa risk (no gene-environment interaction). Three previous candidate gene studies investigated interactions between selenoprotein gene variants and selenium on PCa risk $[19,20,23]$. These studies were on overall PCa and one study showed a significant interaction for a SEPP1 variant [19], and another study for a GPX1 variant [20]. In our study, higher selenium levels were associated with reduced PCa risk independently of common genetic variation in SEPP1 and GPX1. These findings suggest that the beneficial effect of selenium on advanced PCa risk does not depend on common genetic variation in these two selenoprotein genes.

In our study we focused on SEPP1 and GPX1, which according to us are the two most obvious candidate selenoprotein genes to play a role in PCa risk. The human selenoproteome is however encoded by 25 selenoprotein genes [18], and these other genes could also have an effect on PCa risk either directly or by modifying the effect of selenium. In our study, we did not investigate other selenoprotein genes because we could only genotype a small number of SNPs. We therefore believe that it is important for future gene association studies on PCa risk to further this research by investigating genetic variation in other selenoprotein genes $(n=23)$ and examine potential interactions with selenium. Particularly interesting candidate selenoprotein genes are genes encoding other isoforms of GPX (e.g., GPX2, GPX3, GPX4, and GPX6). Our study of GPX1 showed that variants in this gene were associated with advanced PCa risk suggesting that SNPs in other GPX genes may also influence PCa risk. All GPXs have 
similar antioxidant functions that involve reduction of hydroperoxides [26]. Other selenoproteins that may play a role in PCa are the thioredoxin reductases (TXNR). These selenoproteins have a central role in the thioredoxin system, which is important for cellular redox control and signaling [32]. A laboratory study showed that loss of TRXN function because of oxidation and corresponding redox imbalance may play important roles in PCa progression [33]. Some other selenoproteins have biological functions that are however unlikely to play a role in cancer (e.g., iodothyronine deodinases) [18, 34]. While GPX and TXNR have well-known functions potentially involved in cancer, many other selenoproteins have poorly understood biological activities. These include selenoprotein H, I, K, M, O, S, T, V, and W and SEP15 [18, 34]. Further mechanistic research is needed to discover the precise functions of these proteins and identify functional variants in their genes. Furthermore, genes involved in selenoprotein synthesis may also play a role in PCa. The biosynthesis of selenoproteins involves the incorporation of selenocysteine in proteins and this process depends on multiple protein factors such as SECIS-binding protein 2 (SBP2) [18, 34]. Variation in genes encoding these proteins may influence the activity of selenoproteins and might therefore impact PCa risk. Only one previous study investigated genetic variation in this pathway in relation to PCa risk. This small case-control study investigated variation in SBP2 but found no association with PCa risk [24]. Finally, two recent genome-wide association studies investigated common genetic variation in relation to blood selenium levels [35, 36], and the studies identified a number of variants associated with selenium. Although it was not clear to what specific pathways the gene variants belong, they could potentially modify the effect of selenium on (advanced) PCa risk, which would be an interesting topic for a future study.

\subsubsection{Oxidative stress and prostate cancer risk - A role for pro- and antioxidant intake?}

Our study generally supports previous epidemiological research showing that intake of the major antioxidant nutrients vitamin C, vitamin E, $\beta$-carotene, and lycopene is unlikely to influence PCa risk. Our study did however show an association between higher flavonoid intake and reduced advanced PCa risk. Flavonoids are antioxidant polyphenols that include catechins and flavonols and a major dietary source of these components is black tea $[37,38]$. Few other studies examined flavonoids in relation to PCa risk and these studies generally do not support an association [39]. Our study showed that both flavonoid intake and black tea consumption were associated with a reduced risk of advanced PCa but not overall or non-advanced PCa. In our study, flavonoid intake was estimated using a baseline FFQ and a potential limitation is that misclassification may have occurred. Future analyses would therefore benefit from biomarkers of flavonoid intake such as for example urinary markers [40]. Our observation that high black tea consumption reduces PCa risk is supported by a few 
other studies [41, 42]. Most previous investigations of tea and PCa risk, however, exclusively studied overall PCa risk [43], and, additional studies are needed to confirm the inverse association with advanced PCa risk observed in our study.

Based on the existing scientific evidence, an increased intake of well-known antioxidant nutrients such as vitamins and carotenoids is unlikely to reduce PCa risk in a generally healthy population. Antioxidant intake should therefore not be recommended for the prevention of PCa. Future observational studies on (modifiable) risk factors for PCa should focus on exposures other than antioxidant nutrients. It is however not ruled out that some antioxidants may influence PCa risk in specific subgroups such as for example men with a specific genetic background (e.g., oxidative stress-related SNPs; Chapter 7.2.4) or men who have very low baseline antioxidant levels (e.g., selenium; Chapter 7.2.1), and this should be investigated further.

In addition to these antioxidants, there are some well-known pro-oxidant exposures including cigarette smoke, dietary iron, and alcohol [44-46]. These environmental exposures induce the formation of ROS and can thereby cause oxidative stress. Different observational studies investigated these pro-oxidants in relation to PCa risk, but they showed no convincing evidence of an association [47-52], which was confirmed by our study. Although prior research does not suggest that pro-oxidant intake increases PCa risk, exposure to pro-oxidants such as cigarette smoke and alcohol should be limited because they have many known adverse health effects other than the induction of PCa [44].

\section{Pro- and antioxidant intake scores}

In our study we not only examined single pro- and antioxidant exposures but also investigated their combined effects using pro- and antioxidant scores. This may be important because these components act through the same pathway. We created two scores: (1) an antioxidant score (only antioxidants), and (2) an oxidative balance score (both pro- and antioxidants; scored in the opposite way). The scores included major well-known pro- and antioxidant exposures (e.g., antioxidant nutrients, smoking) and were based on previously described scores [45, 46, 53-57]. All score constituents were given an equal weight. In our study, both scores were not associated with PCa risk. Our findings are therefore not consistent with the hypothesis that a higher intake of many antioxidants or presumably beneficial balance between exogenous pro- and antioxidants protects against PCa. A few prior studies investigated comparable proand antioxidant scores in relation to PCa risk and these studies generally do not support an association $[45,58,59]$. We believe that our scores are important measures of pro- and antioxidant intake because they are literature-based and include wellknown pro- and antioxidants (e.g., antioxidant nutrients, smoking). These scores are therefore interesting tools to use in future epidemiological studies on oxidative stress 
and disease risk or prognosis. A potential limitation of our scores is that they are unweighted; all score constituents were giving the same weight. The magnitude of the effects of the pro- and antioxidants may however differ. An alternative approach would be to weigh the individual pro- and antioxidants based on their measured activities. One such approach involves using the ferric-reducing antioxidant potential (FRAP) assay, which measures the reduction of ferric $\left(\mathrm{Fe}^{3+}\right)$ to ferrous ion $\left(\mathrm{Fe}^{2+}\right)$ and has been used to quantify total antioxidants in foods [60]. This approach however also has limitations. One important limitation is that FRAP measures the in vitro antioxidant potential, which may be very different from the in vivo situation. Once metabolized, pro- and antioxidants undergo many complex processes, which probably influence their activities. We therefore believe that a simple unweighted score as used in this thesis is the best available approach to study the combined effects of pro- and antioxidants in epidemiological studies.

\subsubsection{Oxidative stress and prostate cancer - A role for genetic variation?}

Oxidative stress possibly depends on genetic variation in oxidative stress-related genes. Different oxidative stress-related SNPs with potential functional consequences (e.g., altered enzyme activity) have been described [47, 61-66]. These genetic variants may influence PCa risk. Candidate gene association studies have investigated these gene variants in relation to PCa risk, and they showed significant associations with SNPs in different genes [22, 47, 61-69].

In our study, we investigated 14 common genetic variants in major pro- and antioxidant genes that have reported functionality or have been associated with PCa risk before (Table 7.2). We investigated associations between these genetic variants and advanced PCa risk and found one SNP to be associated: CAT rs1001179. This SNP has previously been associated with PCa risk in a small case-control study [69]. The marginal association is biologically plausible because the minor allele of CAT rs1001179, which increased PCa risk in our study, has been associated with reduced catalase activity $[61,70]$. Catalase is an antioxidant enzyme and a reduced catalase enzyme activity may result in deficient antioxidant protection against oxidative stress, which could potentially translate into a higher risk of developing PCa. We also showed marginal associations with two SNPs in the gene encoding the antioxidant enzyme GPX1. These results are discussed in Chapter 7.2.2. None of the other candidate SNPS were associated with advanced PCa risk. This is surprising because we selected candidate SNPs that have been associated with PCa risk in previous studies. The majority of these previous analyses however only examined overall PCa, and the fact that we specifically studied advanced PCa could explain the differences in outcome. Furthermore, many of the previously reported SNP associations were only reported in one or two studies, suggesting that they might have been due to chance. Our findings 
suggest that specific antioxidant gene variants have a main effect on advanced PCa risk. Based on these findings, future gene-association studies are needed to extend this research and more comprehensively study underlying genetic variation. In particular, future studies would benefit from a tagging SNP approach (used in this thesis only for SEPP1 and GPX1) rather than a candidate SNP approach. By only studying specific candidate SNPs, we probably excluded other important genetic variants within the candidate genes that may affect PCa, for example those SNPs that have only been studied to a limited extent and therefore have unknown functional effects. Tagging SNPs allow capturing all common genetic variation in a gene by selecting a small number of SNPs within or near the gene of interest [71]. Given our findings, future studies may want to use tagging SNPs for CAT and GPX genes (i.e., GPX2-6). In our study we found that a CAT variant and two GPX1 variants were associated with advanced PCa risk.

Genome-wide association studies (GWAS) of PCa have not identified risk variants in oxidative stress-related genes [25]. PCa GWAS do not confirm the observed marginal associations with variants in CAT and GPX1 (2 SNPs) in our study. There are a number of possible explanations for these mixed findings. First, while we specially studied advanced stage PCa, most PCa GWAS investigated overall PCa risk. Some recent GWAS have studied more aggressive PCa but these generally used a composite definition that included advanced stage, high grade, and PCa-specific death after additional follow-up $[25,72,73]$. The different definitions of advanced/aggressive PCa may explain the different outcomes. Second, GWAS require stringent P-values for statistical significance to prevent false positive results, which may cause associations with SNPs with weaker P-values to be missed [74]. Third, the candidate SNPs showing a marginal association in our study may have been poorly tagged in PCa GWAS (e.g., $r^{2}<0.9$ ). If GWAS SNPs do not strongly correlate with the actual causal variant, an association could be missed [75]. Fourth, GWAS findings might be biased due to an incorrect control selection approach [76], which may be a problem in many GWAS that use cases and control from different study bases. Fifth, GWAS generally do not consider environmental exposure. Genetic risk variants may interact with the environment and only have an effect on disease risk in subgroups exposed or unexposed to an environmental factor [77].

\section{Gene-environment interactions}

Genetic variants and exposures may have a joint effect on disease risk via gene-environment interaction. The genetic variants may modify potential effects of environmental factors. There have been a few, mostly small, studies that investigated interactions between oxidative stress-related genetic variants and exposures (pro- and 
antioxidants) in relation to PCa risk. These studies produced some statistically significant gene-environment interactions $[47,66,78]$.

In this thesis we investigated interactions between the 14 oxidative stress-related gene variants and 11 pro- and antioxidant exposures in relation to advanced PCa risk. We tested 151 gene-environment interactions in relation to both stage III/IV and IV PCa risk. Because our analysis included a large number of tests $(n=302)$, we decided to adjust for multiple testing using the false discovery rate (FDR) approach [79-81]. We chose this approach because it is less conservative (than for example the Bonferroni method), which is important because our study is hypothesis-based and we, therefore, wanted to limit the probability of excluding true positives. We chose the FDR Q-value threshold of 0.20 , which has been suggested for candidate gene studies [50,51]. A lower threshold is often used in genome-wide association studies, which do not use prior information to select candidate genes [53]. Seven interactions were statistically significant after adjusting for multiple testing (FDR Q-value $<0.20$ ); for stage III/IV PCa these involved intake of $\beta$-carotene (GPX1 rs17650792, hOGG1 rs1052133) and heme iron (GPX1 rs1800668 and rs3448), and for stage IV PCa these involved intake of catechin (SOD2 rs4880) and heme iron (hOGG1 rs1052133, SOD1 rs10432782). These interactions were novel and showed no evidence of a dose-response relationship, i.e., an increase or decrease over exposure categories. We hypothesized that higher antioxidant intake is beneficial (higher pro-oxidant intake is damaging) but did not observe such a risk pattern for any of the 7 statistical interactions. Epidemiological studies have not investigated the reported statistically significant interactions in our study. There is also no mechanistic data that supports the gene-environment interactions. The positive findings in our study therefore require confirmation. When these findings are confirmed, additional mechanistic research is needed to discover the underlying biological mechanism explaining the interactions. A potential limitation of our approach is that we estimated exposure from a single baseline questionnaire (except for selenium where toenail selenium was used for exposure monitoring), which may have resulted in misclassification. Future studies should therefore consider other approaches to monitor environmental exposure. Based on the positive findings in our study, we believe that further research on this topic is needed. In our study we only investigated specific candidates and therefore potentially have excluded important genetic variants in the oxidative stress pathway. Future studies should examine other important SNPs that were not investigated in our study, which may result in novel findings. As discussed previously, future studies on this topic should consider using tagging SNPs for the genes investigated in our study to investigate all common variation within candidate genes. The seven significant interactions in our study involved the genes GPX1, hOGG1, SOD1, and SOD2. Given these findings, future studies should focus on the additional GPX and SOD genes (GPX2-6, SOD3). While GPX 
and SOD are antioxidant enzymes, hOGG1 is involved in oxidative damage repair [66]. We only selected one SNP of this pathway, and future studies may want to investigate this more comprehensively and focus on other gene variants and genes of the oxidative damage repair pathway.

\subsection{Gene-environment interaction and exposure modification by genotype}

Studies of gene-environment interactions aim to describe how genetic and environmental factors jointly influence disease risk [82]. This type of analysis is important because it may provide biological insight and may help to identify genetically susceptible individuals [77]. Many of the examples of gene-environment interactions in the literature are examples of effect or exposure modification [83, 84]. The exposure modifiers are gene variants that act by influencing the function of a biological or metabolic pathway. The gene variants may influence response to a given level of exposure by changing how or how rapidly an exposure is absorbed, processed, or metabolized. A well-known example of exposure modification by genotype involves bladder cancer [85]. The association between cigarette smoking and bladder cancer risk is modified by NAT2, with a higher relative risk from smoking for NAT2 slow acetylators compared to rapid or intermediate acetylators. This interaction is biologically plausible, since aromatic amines, which are thought to be the most important class of bladder carcinogens in tobacco smoke are detoxified by NAT2 [86].

Most gene-environment interaction studies to date are candidate gene studies, which include studies of single genes and exposures and more elaborate investigations of entire pathways including all of the genes and exposures thought to be involved in a particular mechanism [77]. Researchers are beginning to explore the possibility to study gene-environment interactions on a genome-wide scale [77]. Such an approach remains, however, challenging because of the large number of tests that needs to be conducted. The most effective way to apply and detect interactions in the context of GWAS is still unresolved [87]. To date, therefore, most comprehensive studies of gene-environment interactions are hypothesis-based analyses that focus on specific disease pathways.

In our study, we tested for multiplicative gene-environment interactions. This type of interaction occurs when the combined effect of a gene and exposure is larger (or smaller) than the product of their individual effects $[77,88]$. It is believed that it is most appropriate to test for multiplicative interaction when the goal of the study is to investigate disease etiology and when the variables under study affect the same disease mechanism, as was the case in our study [83]. The majority of gene-environment interaction studies test for multiplicative interaction [89]. The interactions are tested by entering a product term into a logistic regression model or Cox regression model (used in our study), which is implicitly exponential and thus 
multiplicative $[88,89]$. Another type of interaction is additive interaction, which occurs when the combined effect of gene and exposure is larger (or smaller) than the sum of their individual effects $[77,88]$. We did not study interaction on an additive scale because there was no convincing a priori evidence that an additive model is more appropriate than a multiplicative model. Identifying the most appropriate model is a challenge in many gene-environment interaction studies and, therefore, future analyses may benefit from approaches that are less dependent on the choice of additive or multiplicative parameterizations [90]. Some researchers believe that it is best to report results of both additive and multiplicative interactions [91]. We decided to not do this in our study because this would result in a very large number of statistical tests $(>600)$ and an associated higher risk of excluding true positives when adjusting for multiple testing. Furthermore, when environmental factors and genetic loci only have modest effects on disease risk, there will not be large differences between additive and multiplicative tests [84]. The factors in our study have relatively modest effects. We investigated single common SNPs, which typically show per-allele odds ratios ranging from 1.10-1.25 [25]. Most pro- and antioxidant exposures in our study also have reported main effects on PCa risk that are generally small $[2,50]$.

\subsection{Concluding remarks}

The thesis adds to an existing body of evidence that a low, suboptimal level of the antioxidant nutrient selenium increases the risk of advanced PCa. The preventive effect of selenium on men with low selenium status remains to be demonstrated in a large randomized controlled trial with PCa risk as the primary outcome of interest, which is required before selenium can be recommended for the prevention of PCa. Scientific evidence does not suggest that other major antioxidants nutrients (vitamins $\mathrm{C}$ and $\mathrm{E}, \boldsymbol{\beta}$-carotene, lycopene) or pro-oxidant environmental factors (smoking, alcohol, heme iron) influence PCa risk. Our study, however, found novel evidence that a higher intake of antioxidant flavonoids is associated with a decreased risk of advanced PCa, but this finding requires confirmation.

Genetic variation in specific disease pathways may influence advanced PCa risk directly or via gene-environment interaction. Our study showed evidence that genetic variation in selenoprotein genes (main effects; no interaction with selenium levels) and oxidative stress-related genes (main effects; interactions with pro- and antioxidant intake) are involved in the development of advanced $\mathrm{PCa}$, suggesting that these pathways play a role in the etiology of PCa. 


\subsection{Recommendations for future research}

Our study on selenium, selenoproteins, oxidative stress, and advanced PCa risk showed several statistically significant findings, which according to us invites further epidemiological research on this topic. We have a number of recommendations for these future analyses.

First, future studies should not only investigate total PCa but also more advanced PCa. Many prior observational studies on PCa risk did not investigate more advanced $P C a$, and the few studies that did found some evidence that risk factors may differ for overall versus advanced PCa. Furthermore, this thesis found that while catechin intake was not associated with overall or non-advanced PCa risk, higher catechin intake was associated with a decreased advanced PCa risk. It is important to study the etiology of advanced PCa because this type of PCa has a poor prognosis and is therefore clinically relevant.

Second, we showed an inverse association between selenium status and advanced PCa risk among men from the Netherlands who have relatively low selenium status. When the association is confirmed, it may be justified to perform a new prevention trial to determine whether (advanced) PCa risk can be reduced by increasing selenium status from suboptimal to optimal. Importantly, such a trial should focus on men from regions where selenium levels are low (e.g., European populations) and not on men who are selenium-replete (US men) because additional selenium is not expected to have a beneficial effect when baseline selenium is already adequate.

Third, we showed that genetic variants in selenoprotein genes and genes related to oxidative stress influence advanced PCa risk. Our study utilizes a candidate gene approach and we may therefore have excluded important candidates. Future investigations are needed to further this research and study the role of additional potentially important candidate gene(s) (variants) in these pathways that were not examined in this thesis. These analyses would benefit from a tagging SNP approach, which allows capturing all common genetic variation in genes.

\section{References}

1. van den Brandt PA, Zeegers MP, Bode $P$, et al. Toenail selenium levels and the subsequent risk of prostate cancer: a prospective cohort study. Cancer epidemiology, biomarkers \& prevention : a publication of the American Association for Cancer Research, cosponsored by the American Society of Preventive Oncology 2003;12(9):866-871.

2. Hurst R, Hooper L, Norat $T$, et al. Selenium and prostate cancer: systematic review and meta-analysis. The American journal of clinical nutrition 2012;96(1):111-122.

3. Clark LC, Combs GF, Turnbull BW, et al. Effects of selenium supplementation for cancer prevention in patients with carcinoma of the skin a randomized controlled trial - A randomized controlled trial. JamaJournal of the American Medical Association 1996;276(24):1957-1963.

4. Klein EA, Thompson IM, Jr., Tangen CM, et al. Vitamin E and the risk of prostate cancer: the Selenium and Vitamin E Cancer Prevention Trial (SELECT). JAMA : the journal of the American Medical Association 2011;306(14):1549-1556. 
5. Duffield-Lillico AJ, Dalkin BL, Reid ME, et al. Selenium supplementation, baseline plasma selenium status and incidence of prostate cancer: an analysis of the complete treatment period of the Nutritional Prevention of Cancer Trial. BJU international 2003;91(7):608-612.

6. Lippman SM, Klein EA, Goodman PJ, et al. Effect of selenium and vitamin E on risk of prostate cancer and other cancers: the Selenium and Vitamin E Cancer Prevention Trial (SELECT). JAMA : the journal of the American Medical Association 2009;301(1):39-51.

7. Burk RF, Hill KE. Selenoprotein P-Expression, functions, and roles in mammals. Biochimica Et Biophysica Acta-General Subjects 2009;1790(11):1441-1447.

8. Hurst R, Collings R, Harvey $L$, et al. EURRECA-Estimating Selenium Requirements for Deriving Dietary Reference Values. Critical Reviews in Food Science and Nutrition 2013;53(10):1077-1096.

9. Duffield AJ, Thomson CD, Hill KE, et al. An estimation of selenium requirements for New Zealanders. The American journal of clinical nutrition 1999;70(5):896-903.

10. Rayman MP. Selenoproteins and human health: insights from epidemiological data. Biochimica et biophysica acta 2009;1790(11):1533-1540.

11. Hurst R, Armah CN, Dainty JR, et al. Establishing optimal selenium status: results of a randomized, double-blind, placebo-controlled trial. The American journal of clinical nutrition 2010;91(4):923-931.

12. Kristal AR, Darke AK, Morris JS, et al. Baseline Selenium Status and Effects of Selenium and Vitamin E Supplementation on Prostate Cancer Risk. Journal of the National Cancer Institute 2014.

13. Fairweather-Tait SJ, Bao YP, Broadley MR, et al. Selenium in Human Health and Disease. Antioxidants \& redox signaling 2011;14(7):1337-1383.

14. Rayman MP. Selenium and human health. Lancet 2012;379(9822):1256-1268.

15. Slotnick MJ, Nriagu JO. Validity of human nails as a biomarker of arsenic and selenium exposure: $A$ review. Environmental research 2006;102(1):125-139.

16. Waters DJ, Shen S, Glickman LT, et al. Prostate cancer risk and DNA damage: translational significance of selenium supplementation in a canine model. Carcinogenesis 2005;26(7):1256-1262.

17. Goyal A, Terry MB, Siegel AB. Serum antioxidant nutrients, vitamin a, and mortality in u.s. Adults. Cancer epidemiology, biomarkers \& prevention : a publication of the American Association for Cancer Research, cosponsored by the American Society of Preventive Oncology 2013;22(12):2202-2211.

18. Papp LV, Lu J, Holmgren A, et al. From selenium to selenoproteins: synthesis, identity, and their role in human health. Antioxidants \& redox signaling 2007;9(7):775-806.

19. Penney KL, Li H, Mucci LA, et al. Selenoprotein $P$ genetic variants and mrna expression, circulating selenium, and prostate cancer risk and survival. The Prostate 2013;73(7):700-705.

20. Steinbrecher A, Meplan C, Hesketh J, et al. Effects of selenium status and polymorphisms in selenoprotein genes on prostate cancer risk in a prospective study of European men. Cancer epidemiology, biomarkers \& prevention : a publication of the American Association for Cancer Research, cosponsored by the American Society of Preventive Oncology 2010;19(11):2958-2968.

21. Arsova-Sarafinovska Z, Matevska N, Eken A, et al. Glutathione peroxidase 1 (GPX1) genetic polymorphism, erythrocyte GPX activity, and prostate cancer risk. International urology and nephrology 2009;41(1):63-70.

22. Geybels MS, Hutter CM, Kwon EM, et al. Variation in selenoenzyme genes and prostate cancer risk and survival. The Prostate 2013;73(7):734-742.

23. Penney KL, Schumacher FR, Li H, et al. A large prospective study of SEP15 genetic variation, interaction with plasma selenium levels, and prostate cancer risk and survival. Cancer prevention research 2010;3(5):604-610.

24. Meplan C, Rohrmann S, Steinbrecher A, et al. Polymorphisms in thioredoxin reductase and selenoprotein $\mathrm{K}$ genes and selenium status modulate risk of prostate cancer. PloS one 2012;7(11):e48709.

25. Eeles RA, Olama AA, Benlloch S, et al. Identification of 23 new prostate cancer susceptibility loci using the iCOGS custom genotyping array. Nature genetics 2013;45(4):385-391, 391e381-382.

26. Brigelius-Flohe R, Kipp A. Glutathione peroxidases in different stages of carcinogenesis. Biochimica et biophysica acta 2009;1790(11):1555-1568.

27. Gonzalez-Moreno O, Boque N, Redrado M, et al. Selenoprotein-P is down-regulated in prostate cancer, which results in lack of protection against oxidative damage. The Prostate 2011;71(8):824-834.

28. Meyer HA, Hollenbach B, Stephan C, et al. Reduced serum selenoprotein $P$ concentrations in German prostate cancer patients. Cancer epidemiology, biomarkers \& prevention : a publication of the American 
Association for Cancer Research, cosponsored by the American Society of Preventive Oncology 2009;18(9):2386-2390.

29. Meplan C, Nicol F, Burtle BT, et al. Relative abundance of selenoprotein P isoforms in human plasma depends on genotype, se intake, and cancer status. Antioxidants \& redox signaling 2009;11(11):26312640.

30. Foster CB, Aswath K, Chanock SJ, et al. Polymorphism analysis of six selenoprotein genes: support for a selective sweep at the glutathione peroxidase 1 locus (3p21) in Asian populations. BMC genetics 2006; 7:56.

31. Bhatti P, Stewart PA, Hutchinson A, et al. Lead exposure, polymorphisms in genes related to oxidative stress, and risk of adult brain tumors. Cancer epidemiology, biomarkers \& prevention : a publication of the American Association for Cancer Research, cosponsored by the American Society of Preventive Oncology 2009;18(6):1841-1848.

32. Gromer S, Urig S, Becker K. The thioredoxin system--from science to clinic. Med Res Rev 2004;24(1):4089.

33. Shan $\mathrm{W}$, Zhong $\mathrm{W}$, Zhao $\mathrm{R}$, et al. Thioredoxin 1 as a subcellular biomarker of redox imbalance in human prostate cancer progression. Free radical biology \& medicine 2010;49(12):2078-2087.

34. Hatfield DL, Tsuji PA, Carlson BA, et al. Selenium and selenocysteine: roles in cancer, health, and development. Trends Biochem Sci 2014.

35. Evans DM, Zhu G, Dy V, et al. Genome-wide association study identifies loci affecting blood copper, selenium and zinc. Human molecular genetics 2013;22(19):3998-4006.

36. Gong J, Hsu L, Harrison T, et al. Genome-wide association study of serum selenium concentrations. Nutrients 2013;5(5):1706-1718.

37. Pietta PG. Flavonoids as antioxidants. J Nat Prod 2000;63(7):1035-1042.

38. Ross JA, Kasum CM. Dietary flavonoids: bioavailability, metabolic effects, and safety. Annu Rev Nutr 2002;22:19-34.

39. Neuhouser ML. Dietary flavonoids and cancer risk: evidence from human population studies. Nutrition and cancer 2004;50(1):1-7.

40. Bredsdorff L, Obel T, Dethlefsen C, et al. Urinary flavonoid excretion and risk of acute coronary syndrome in a nested case-control study. The American journal of clinical nutrition 2013;98(1):209-216.

41. Geybels MS, Neuhouser ML, Stanford JL. Associations of tea and coffee consumption with prostate cancer risk. Cancer causes \& control : CCC 2013;24(5):941-948.

42. Kurahashi N, Sasazuki S, Iwasaki M, et al. Green tea consumption and prostate cancer risk in Japanese men: a prospective study. American journal of epidemiology 2008;167(1):71-77.

43. Lin YW, Hu ZH, Wang $X$, et al. Tea consumption and prostate cancer: an updated meta-analysis. World J Surg Oncol 2014;12:38.

44. Valko M, Rhodes CJ, Moncol J, et al. Free radicals, metals and antioxidants in oxidative stress-induced cancer. Chemico-biological interactions 2006;160(1):1-40.

45. Agalliu I, Kirsh VA, Kreiger N, et al. Oxidative balance score and risk of prostate cancer: results from a case-cohort study. Cancer Epidemiol 2011;35(4):353-361.

46. Goodman M, Bostick RM, Dash C, et al. A summary measure of pro- and anti-oxidant exposures and risk of incident, sporadic, colorectal adenomas. Cancer causes \& control : CCC 2008;19(10):1051-1064.

47. Choi JY, Neuhouser ML, Barnett MJ, et al. Iron intake, oxidative stress-related genes (MnSOD and MPO) and prostate cancer risk in CARET cohort. Carcinogenesis 2008;29(5):964-970.

48. Jakszyn PG, Allen NE, Lujan-Barroso L, et al. Nitrosamines and heme iron and risk of prostate cancer in the European prospective investigation into cancer and nutrition. Cancer epidemiology, biomarkers \& prevention : a publication of the American Association for Cancer Research, cosponsored by the American Society of Preventive Oncology 2012;21(3):547-551.

49. Rota M, Scotti L, Turati F, et al. Alcohol consumption and prostate cancer risk: a meta-analysis of the dose-risk relation. Eur J Cancer Prev 2012;21(4):350-359.

50. Vance TM, Su J, Fontham ET, et al. Dietary antioxidants and prostate cancer: a review. Nutrition and cancer 2013;65(6):793-801.

51. Zu K, Giovannucci E. Smoking and aggressive prostate cancer: a review of the epidemiologic evidence. Cancer causes \& control : CCC 2009;20(10):1799-1810.

52. Huncharek M, Haddock KS, Reid R, et al. Smoking as a risk factor for prostate cancer: a meta-analysis of 24 prospective cohort studies. Am J Public Health 2010;100(4):693-701. 
53. Goodman M, Bostick RM, Dash C, et al. Hypothesis: oxidative stress score as a combined measure of pro-oxidant and antioxidant exposures. Annals of epidemiology 2007;17(5):394-399.

54. Li H, Kantoff PW, Giovannucci E, et al. Manganese superoxide dismutase polymorphism, prediagnostic antioxidant status, and risk of clinical significant prostate cancer. Cancer research 2005;65(6):24982504.

55. Terry $\mathrm{P}$, Lagergren J, Ye W, et al. Antioxidants and cancers of the esophagus and gastric cardia. International journal of cancer. Journal international du cancer 2000;87(5):750-754.

56. Van Hoydonck PG, Temme EH, Schouten EG. A dietary oxidative balance score of vitamin C, betacarotene and iron intakes and mortality risk in male smoking Belgians. The Journal of nutrition 2002;132(4):756-761.

57. Wright ME, Mayne ST, Stolzenberg-Solomon RZ, et al. Development of a comprehensive dietary antioxidant index and application to lung cancer risk in a cohort of male smokers. American journal of epidemiology 2004;160(1):68-76.

58. Goodman M, Bostick RM, Gross M, et al. Combined measure of pro- and anti-oxidant exposures in relation to prostate cancer and colorectal adenoma risk: an update. Annals of epidemiology 2010;20(12):955-957.

59. Lakkur S, Goodman M, Bostick RM, et al. Oxidative balance score and risk for incident prostate cancer in a prospective U.S. cohort study. Annals of epidemiology 2014.

60. Russnes KM, Wilson KM, Epstein MM, et al. Total antioxidant intake in relation to prostate cancer incidence in the Health Professionals Follow-Up Study. International journal of cancer. Journal international du cancer 2014;134(5):1156-1165.

61. Ahn J, Nowell S, McCann SE, et al. Associations between catalase phenotype and genotype: modification by epidemiologic factors. Cancer epidemiology, biomarkers \& prevention : a publication of the American Association for Cancer Research, cosponsored by the American Society of Preventive Oncology 2006;15(6):1217-1222.

62. Cheng TY, Barnett MJ, Kristal AR, et al. Genetic variation in myeloperoxidase modifies the association of serum alpha-tocopherol with aggressive prostate cancer among current smokers. The Journal of nutrition 2011;141(9):1731-1737.

63. Kang D, Lee KM, Park SK, et al. Functional variant of manganese superoxide dismutase (SOD2 V16A) polymorphism is associated with prostate cancer risk in the prostate, lung, colorectal, and ovarian cancer study. Cancer epidemiology, biomarkers \& prevention : a publication of the American Association for Cancer Research, cosponsored by the American Society of Preventive Oncology 2007;16(8):15811586.

64. Lee KM, Kang D, Park SK, et al. Nitric oxide synthase gene polymorphisms and prostate cancer risk. Carcinogenesis 2009;30(4):621-625.

65. Stevens VL, Rodriguez C, Talbot JT, et al. Paraoxonase 1 (PON1) polymorphisms and prostate cancer in the CPS-II Nutrition Cohort. The Prostate 2008;68(12):1336-1340.

66. Zhang J, Dhakal IB, Greene G, et al. Polymorphisms in hOGG1 and XRCC1 and risk of prostate cancer: effects modified by plasma antioxidants. Urology 2010;75(4):779-785.

67. Abe M, Xie W, Regan MM, et al. Single-nucleotide polymorphisms within the antioxidant defence system and associations with aggressive prostate cancer. BJU international 2011;107(1):126-134

68. Geybels MS, van den Brandt PA, Schouten $L$, et al. Selenoprotein gene variants, toenail selenium levels, and risk for advanced prostate cancer. Journal of the National Cancer Institute 2014;106(3):dju003.

69. Tefik T, Kucukgergin C, Sanli O, et al. Manganese superoxide dismutase Ile58Thr, catalase C-262T and myeloperoxidase G-463A gene polymorphisms in patients with prostate cancer: relation to advanced and metastatic disease. BJU international 2013;112(4):E406-414.

70. Ahn J, Gammon MD, Santella RM, et al. Associations between breast cancer risk and the catalase genotype, fruit and vegetable consumption, and supplement use. American journal of epidemiology 2005;162(10):943-952.

71. Carlson CS, Eberle MA, Rieder MJ, et al. Selecting a maximally informative set of single-nucleotide polymorphisms for association analyses using linkage disequilibrium. American journal of human genetics 2004;74(1):106-120.

72. FitzGerald LM, Kwon EM, Conomos MP, et al. Genome-wide association study identifies a genetic variant associated with risk for more aggressive prostate cancer. Cancer epidemiology, biomarkers \& prevention : a publication of the American Association for Cancer Research, cosponsored by the American Society of Preventive Oncology 2011;20(6):1196-1203. 
73. Amin Al Olama A, Kote-Jarai Z, Schumacher FR, et al. A meta-analysis of genome-wide association studies to identify prostate cancer susceptibility loci associated with aggressive and non-aggressive disease. Human molecular genetics 2013;22(2):408-415.

74. Shiels MS, Engels EA, Shi J, et al. Genetic variation in innate immunity and inflammation pathways associated with lung cancer risk. Cancer 2012;118(22):5630-5636.

75. Gusev A, Bhatia G, Zaitlen N, et al. Quantifying missing heritability at known GWAS loci. PLoS genetics 2013;9(12):e1003993.

76. Fradin DD, Fallin MD. Influence of control selection in genome-wide association studies: the example of diabetes in the Framingham Heart Study. BMC proceedings 2009;3 Suppl 7:S113.

77. Thomas D. Gene--environment-wide association studies: emerging approaches. Nature reviews. Genetics 2010;11(4):259-272.

78. Li H, Kantoff PW, Giovannucci E, et al. Manganese superoxide dismutase polymorphism, prediagnostic antioxidant status, and risk of clinical significant prostate cancer. Cancer research 2005;65(6):24982504.

79. Benjamini Y, Hochberg Y. Controlling the False Discovery Rate: a Practical and Powerful Approach to Multiple Testing. J. R. Statist. Soc. 1995;57(1):289-300.

80. Karami S, Andreotti G, Koutros S, et al. Pesticide Exposure and Inherited Variants in Vitamin D Pathway Genes in Relation to Prostate Cancer. Cancer epidemiology, biomarkers \& prevention : a publication of the American Association for Cancer Research, cosponsored by the American Society of Preventive Oncology 2013.

81. Smith NL, Hindorff LA, Heckbert SR, et al. Association of genetic variations with nonfatal venous thrombosis in postmenopausal women. Jama-Journal of the American Medical Association 2007;297(5):489-498.

82. Hunter DJ. Gene-environment interactions in human diseases. Nature reviews. Genetics 2005;6(4):287298.

83. Christiani DC. Invited commentary: the action in the interaction and exposure modification. American journal of epidemiology 2012;175(7):606-608.

84. Weinberg CR. Interaction and exposure modification: are we asking the right questions? American journal of epidemiology 2012;175(7):602-605.

85. Garcia-Closas M, Malats N, Silverman D, et al. NAT2 slow acetylation, GSTM1 null genotype, and risk of bladder cancer: results from the Spanish Bladder Cancer Study and meta-analyses. Lancet 2005;366(9486):649-659.

86. Hein DW. Molecular genetics and function of NAT1 and NAT2: role in aromatic amine metabolism and carcinogenesis. Mutat Res 2002;506-507:65-77.

87. Cornelis MC, Tchetgen EJ, Liang L, et al. Gene-environment interactions in genome-wide association studies: a comparative study of tests applied to empirical studies of type 2 diabetes. American journal of epidemiology 2012;175(3):191-202.

88. Knol MJ, van der Tweel I, Grobbee DE, et al. Estimating interaction on an additive scale between continuous determinants in a logistic regression model. International journal of epidemiology 2007;36(5):1111-1118.

89. Ahlbom A, Alfredsson L. Interaction: A word with two meanings creates confusion. European journal of epidemiology 2005;20(7):563-564.

90. Hutter CM, Mechanic LE, Chatterjee N, et al. Gene-environment interactions in cancer epidemiology: a National Cancer Institute Think Tank report. Genetic epidemiology 2013;37(7):643-657.

91. Knol MJ, VanderWeele TJ. Recommendations for presenting analyses of effect modification and interaction. International journal of epidemiology 2012;41(2):514-520.

92. Duffield-Lillico AJ, Reid ME, Turnbull BW, et al. Baseline characteristics and the effect of selenium supplementation on cancer incidence in a randomized clinical trial: a summary report of the Nutritional Prevention of Cancer Trial. Cancer epidemiology, biomarkers \& prevention : a publication of the American Association for Cancer Research, cosponsored by the American Society of Preventive Oncology 2002;11(7):630-639. 

Summary / Samenvatting 


\section{Summary}

Prostate cancer (PCa) is a common disease that is clinically heterogeneous. Only a subset of all PCa patients has advanced disease that is associated with a poor prognosis. Many other PCa patients have relatively slow-growing tumors that may never become clinically relevant when left untreated. The present thesis deals with PCa risk factors and specifically focuses on advanced PCa. Advanced PCa may have a different etiology compared to non-advanced PCa. Many previous studies on PCa risk factors only focused on overall PCa. In the present thesis, we investigated the role of selenium and the related oxidative stress pathway, which according to previous scientific research may play a role in (advanced) PCa development. Oxidative stress is a biological condition that results from an imbalance between reactive oxygen species and antioxidants in favor of the former. It has been hypothesized that oxidative stress is involved in aging and age-related diseases including PCa. Selenium is an essential micronutrient with antioxidant properties. The oxidative stress pathway includes many endogenous (genetic) and exogenous (environmental) pro- and antioxidants.

The various analyses in this thesis were conducted within the large prospective Netherlands Cohort Study, which included 58,279 men at baseline in September 1986. Netherlands Cohort Study participants completed a baseline questionnaire on many potential cancer risk factors including pro- and antioxidant intake. Study participants also provided toenail clippings, which were used to measure selenium concentrations and to isolate DNA for genotyping. We used a candidate gene association approach and studied single nucleotide polymorphisms (SNPs), which are the most common type of genetic variation in humans. We studied SNPs in genes encoding major selenoproteins (through which selenium exerts its biological activities) and oxidative stress-related proteins (e.g., pro- and antioxidant enzymes). Cancer cases were prospectively identified by annual record linkage to cancer registries. For the research described in this thesis, a follow-up of 17.3 years was used (1986-2003). Clinical and pathological TNM (Tumor, Node, Metastasis) stage was used to classify PCa. Two definitions of advanced PCa were used, i.e.: stage III/IV PCa (T3-4, N+, or M1) and stage IV PCa (T4, N+, or M1). The Netherlands Cohort Study employs a case-cohort design, which involves using a subcohort to estimate the person-time experience. The advantage of this design is that it considerably reduces data collection, which is important because costly and time-consuming methods for exposure monitoring and genotyping were used in the thesis.

In Chapter $\mathbf{2}$ of the thesis we investigated the association between toenail selenium levels and advanced PCa risk. Toenail selenium is a selenium status biomarker that reflects long-term selenium intake (compared to blood selenium), and our study was the first large study of toenail selenium and advanced PCa risk. In our 
study population of men from the Netherlands with low to moderate selenium status, we found that toenail selenium levels were inversely associated with advanced PCa risk. An inverse association between selenium status and advanced PCa risk has been shown previously in several other prospective studies that used blood selenium for exposure monitoring. Future research on selenium and PCa risk should focus on men who have low, suboptimal selenium levels (e.g., European men).

In Chapter $\mathbf{3}$ we studied the associations between common genetic variants in the major selenoprotein genes SEPP1 (5 tagging SNPs) and GPX1 (3 tagging SNPs) and advanced PCa risk and examined potential gene-environment interactions with toenail selenium levels. The study showed that SEPP1 and GPX1 gene variants influenced advanced PCa risk (marginal associations) but the variants did not modify the association with toenail selenium (no gene-environment interactions). The positive findings for SEPP1 and GPX1 in our study suggest that selenoprotein genes may play a role in the etiology of advanced $P C$.

In Chapters $\mathbf{4}$ and $\mathbf{5}$ of the thesis we studied pro- and antioxidant intake in relation to overall and advanced PCa risk. The study showed that most antioxidant nutrients (e.g., vitamins, carotenoids) and pro-oxidant exposures (e.g., smoking, alcohol), either separately or combined in a score, were not associated. This is supported by previous research on this topic. However, our study also showed that intake of antioxidant flavonoids and black tea (a major source of flavonoids) was associated with a reduced risk of advanced but not total PCa. Few other studies examined flavonoids in relation to (advanced) PCa risk and these studies generally do not support an association. Our findings therefore require confirmation from other studies.

In Chapter 6 we investigated associations between SNPs in oxidative stressrelated genes and advanced PCa risk and examined potential interactions with proand antioxidant intake. The tested SNPs $(n=14)$ were candidate SNPs that have reported functionality or have been associated with PCa risk in previous studies. We found that a SNP in the antioxidant gene CAT (rs1001179) was associated with advanced PCa risk. The association has been reported in one previous study (for overall PCa) and is supported by functional data. Furthermore, we found seven novel geneenvironment interactions that retained significance after adjusting for multiple testing. The statistically significant multiplicative gene-environment interactions in our study suggest that the oxidative stress pathway is involved in the etiology of advanced PCa. We therefore think that additional epidemiological research on this topic is needed.

In Chapter 7, the main study findings as well as the study limitations are discussed. Furthermore, recommendations for future studies are given. 


\section{Samenvatting}

Prostaatkanker is een veelvoorkomende ziekte waarvan uiteindelijk slechts een subgroep advanced (d.i., gevorderd stadium) zal worden. Advanced prostaatkanker is een type prostaatkanker dat geassocieerd is met een slechte prognose. Deze thesis gaat over risicofactoren voor advanced prostaatkanker. Wij onderzochten de mogelijke rol van selenium en de gerelateerde oxidatieve stress pathway. Oxidatieve stress is een biologische toestand die het gevolg is van een verstoorde balans tussen schadelijke reactieve zuurstofvormen (meer) en antioxidanten (minder). Oxidatieve stress is mogelijk betrokken bij veroudering en ouderdomsgerelateerde aandoeningen zoals prostaatkanker. Selenium is een essentieel micronutriënt en antioxidant. De oxidatieve stress pathway omvat verschillende endogene (genetische) en exogene (omgevingsfactoren) pro- en antioxidanten.

De analyses beschreven in deze thesis zijn uitgevoerd in de prospectieve Nederlandse Cohortstudie (58279 mannen bij baseline in 1986). De studiedeelnemers hebben aan het begin van de studie een gedetailleerde vragenlijst (o.a. over dieet en andere leefstijlfactoren) ingevuld. Daarnaast hebben ongeveer $80 \%$ van de deelnemers een teennagelmonster aangeleverd. Dit biologisch materiaal werd in dit onderzoek gebruikt voor (1) het bestuderen van genetische variatie en (2) de bepaling van seleniumconcentraties. Wij onderzochten genetische variatie in belangrijke selenoproteïnegenen (de activiteit van selenium wordt uitgeoefend door deze genen) en genen van de oxidatieve stress pathway. Voor dit onderzoek werden de deelnemers van de studie 17.3 jaar gevolgd (1986-2003) voor het ontstaan van advanced (stadium III/IV of IV) prostaatkanker. De Nederlandse Cohortstudie maakt gebruik van het casecohort design.

In Hoofdstuk $\mathbf{2}$ van de thesis onderzochten wij de associatie tussen teennagelseleniumwaarden en het risico op advanced prostaatkanker. Wij vonden dat een lage seleniumwaarde geassocieerd was met een sterk verlaagd risico op advanced prostaatkanker.

In Hoofdstuk 3 bestudeerden wij de associaties tussen (tagging) genetische varianten in twee selenoproteïnegenen, d.i., SEPP1 en GPX1, en advanced prostaatkanker. Wij onderzochten tevens mogelijke gen-omgevingsinteracties met teennagelselenium. De studie toonde aan dat het risico op advanced prostaatkanker werd beïnvloed door de genetische varianten. De associatie tussen teennagelseleniumwaarden en advanced prostaatkanker werd echter niet gemodificeerd door de genetische varianten (d.i., geen gen-omgevingsinteractie).

In Hoofdstuk $\mathbf{4}$ en $\mathbf{5}$ van de thesis bestudeerden wij de inname van pro- en antioxidanten in relatie tot totaal en advanced prostaatkanker. De meeste pro- en antioxidanten waren niet geassocieerd, d.i., als individuele factor of gecombineerd in 
een score. Wij vonden wel dat een hogere inname van flavonoïden (een groep antioxidanten) geassocieerd was met een verlaagd risico op advanced prostaatkanker. Deze bevinding heeft nood aan bevestiging van andere studies.

In Hoofdstuk 6 onderzochten wij de associaties tussen (kandidaat) genetische varianten in oxidatieve stress-gerelateerde genen en advanced prostaatkanker en bestudeerden potentiële interacties met inname van pro- en antioxidanten. Wij vonden dat een genetische variant in het catalase-gen (CAT) geassocieerd was met het risico op advanced prostaatkanker en identificeerden zeven statistisch significante gen-omgevingsinteracties.

Hoofdstuk 7 omvat een wetenschappelijke discussie, mogelijke beperkingen van het onderzoek, en aanbevelingen voor toekomstig onderzoek. 

Knowledge Valorization 
Knowledge valorization refers to the utilization of scientific knowledge in practice. This section describes how the scientific findings in this thesis may have an impact on society or economy. The different study findings and their potential opportunities for knowledge valorization are discussed separately.

\section{Selenium}

Selenium is an essential micronutrient that may prevent the development of (advanced) prostate cancer (PCa). We conducted a prospective analysis to investigate the association between toenail selenium levels (a selenium status biomarker) and advanced PCa risk in a population of men from the Netherlands with low to moderate selenium status. Our study showed that higher toenail selenium levels were associated with a substantial decrease in risk of advanced PCa. The association is supported by other prospective studies that used blood selenium for exposure monitoring [1]. Previous prospective studies showed that the inverse association with selenium exists for both overall and advanced PCa, but is slightly more pronounced for advanced PCa. Despite the evidence from observational studies, selenium cannot be recommended for the prevention of PCa before such an effect has been demonstrated in a randomized controlled clinical trial with PCa risk as the primary outcome of interest. Designing and conducting such a trial could therefore be an important next step in this research. The trial should clearly focus on men from regions where selenium soil concentrations (and hence dietary selenium intake and status) are low (e.g., European men).

If the anticancer effect of selenium has been demonstrated in a clinical trial and selenium can be used to reduce PCa risk, this would have important public health and economic consequences. PCa is the most common cancer in men. In the Netherlands in 2011, 11,428 men were diagnosed with PCa and 2,500 men died as a result of the disease [2]. According to data from the Netherlands National Institute for Public Health and the Environment the total costs for PCa in the Netherlands in 2011 were 254 million Euro [3]. Furthermore, compared to other common cancers (e.g., breast, lung) PCa has no known modifiable risk factors $[4,5]$. What is particularly important is that evidence supports that selenium may not only prevent total PCa but also more advanced PCa. This type of PCa is associated with a poor prognosis [6], and is therefore the most important type of PCa when it comes to prevention. While non-advanced prostate cancers have a 5 -year survival rate of nearly $100 \%$, this is considerably reduced for advanced PCa (45\% for stage IV PCa) [7]. In addition, low selenium intake is common in many regions worldwide [8], suggesting that there is a very large target population that would potentially benefit from additional selenium intake. In addition, while our study focused on PCa, low-selenium is believed to be a potential risk factor 
for many other chronic diseases (e.g., other cancers, cardiovascular disease) [8]. This further highlights the social and economic relevance of counteracting the problem of low selenium intake.

A number of strategies can potentially be used to increase selenium intake. These strategies should focus on men from regions with low selenium soil concentrations who therefore have a low selenium intake and status (e.g., European men). The simplest and safest strategy to increase selenium intake is to consume more foods that are rich sources of selenium (e.g., specific types of fish, meat, nuts). Identifying those foods can be done by dietitians who then could develop specialized dietary recommendations and guidelines for low-selenium men. Further research is needed to design and optimize such dietary interventions. Selenium intake can also be increased by using selenium supplements. However, it is important to note that high-dose supplementation may cause harm in some situations. This has also been demonstrated for selenium [9]. It will therefore be important that the industry develops low-dose selenium supplements that are safe to use by men from low-selenium regions and that efficiently increase selenium status from suboptimal to optimal but not greater than optimal. Another possibility to indirectly increase selenium intake is to increase selenium soil concentrations in low selenium regions. Higher soil selenium levels will result in higher selenium levels in the foods grown on these soils. This has been successfully done in Finland in the 1980s by using selenium enriched fertilizers [10]. Therefore, policy makers in other countries with low selenium soil concentrations may consider using a similar strategy to increase selenium soil levels and the associated selenium intake in these regions.

\section{Selenoprotein gene variants}

Selenium exerts its biological activity through selenoproteins. The human selenoproteome is encoded by 25 known selenoprotein genes [11]. We conducted a gene association study to investigate how genetic variants in selenoprotein genes influence advanced PCa risk directly or by modifying the effect of selenium. Genetic variants in these genes may influence the activity of selenoproteins. We focused on two selenoprotein genes, i.e., SEPP1 (involved in selenium transport) and GPX1 (involved in antioxidant protection). Our study showed marginal associations for variants in both genes, suggesting a role for these genes, and the pathways they are involved in, in advanced PCa development. Genetic variation in the genes did, however, not modify the association with toenail selenium levels (no geneenvironment interaction). There have been few other studies that investigated selenoprotein genes in relation to selenium status and advanced PCa risk. 
Gene-environment interaction research is primarily focused on understanding the etiology of a disease. Knowledge valorization is no primary aim of this research and there are therefore no immediate opportunities to use the findings from these studies to create value. One potential long-term opportunity to use gene-environment interactions in practice is to identify genetically susceptible individuals. For example, genetic variation in selenoprotein genes may influence the activity of selenoproteins and therefore how selenium intake affects advanced PCa risk. Specific selenoprotein gene variants may cause certain individuals to become more susceptible to low selenium status. If that is the case, selenoprotein gene variants can be used to identify those genetically susceptible individuals that are expected to benefit (more) from additional selenium intake. The gene variants could then potentially be used as a tool by medical specialists, for example to make recommendations or dietary guidelines on selenium supplementation. The gene variants may also be used by researchers planning a future trial on selenium supplementation and PCa risk. Although our study indicated that the effect of selenium on advanced PCa risk was independent of SEPP1 and GPX1 common variation, other selenoprotein genes may still play a role. Therefore, further gene association studies are needed to investigate the potential role of other selenoprotein genes $(n=23)$ not investigated in our study. It is important to note that identifying genetically susceptible individuals using gene-environment interactions will require more research on a number of questions related to feasibility, costs, ethical issues, etcetera; before such findings can be used in practice.

\section{Flavonoids}

Our prospective study showed that a higher intake of antioxidant flavonoids (catechins and flavonols) was associated with a reduced risk of advanced PCa, but not overall or non-advanced PCa. Few other studies examined flavonoids in relation to (advanced) PCa risk and these studies generally do not support an association. Our findings therefore require confirmation from other studies. We also investigated the associations of (intake of) other antioxidant nutrients (e.g., vitamins, carotenoids) and pro-oxidant exposures (i.e., smoking). Our study showed no evidence of an association of these exposures, which was confirmed by previous epidemiological research [12, 13].

It is important that we do research on dietary and other lifestyle factors in relation to PCa risk. PCa is a common cancer with no established modifiable risk factors (unlike other common cancers) [4, 5]. According to the most recent data approximately 1 in 7 US men will develop PCa during his lifetime. A potential preventive effect of flavonoid intake could therefore help reduce the enormous social and economic impact of PCa (also see section on selenium and PCa risk, page 148- 
149). What is particularly interesting is that the inverse association with flavonoid intake in our study was specifically for advanced PCa. This type of PCa is clinically relevant because it has a poor prognosis [6], unlike non-advanced PCa. Advanced PCa is therefore the most important type of PCa when it comes to prevention.

Our finding that flavonoids may reduce the risk of advanced PCa requires confirmation from other studies, and flavonoids cannot be recommended for the prevention of PCa before such an effect has been demonstrated in a large clinical trial. Assuming our findings were to be confirmed; how can flavonoids be used to reduce the incidence of advanced PCa? One simple strategy would be to recommend men with a low flavonoid intake to consume more foods that are rich sources of flavonoids. These foods include tea, apples, onions, chocolate, legumes, cabbages, and leafy vegetables. Dietitians could develop dietary guidelines/recommendations for these men to increase their flavonoid intake/status to an optimal level. Further research is needed to determine how this can exactly be achieved. Additional research is also needed to determine the subgroups that are most likely to benefit from such an intervention. Furthermore, our finding that flavonoids were inversely associated with advanced but not overall or non-advanced PCa risk suggests that flavonoids may reduce the progression of $\mathrm{PCa}$. If that is the case, flavonoids can potentially be used for the secondary prevention of $\mathrm{PCa}$, i.e., preventing PCa progression (e.g., prostatespecific antigen (PSA) progression, biochemical recurrence, metastasis, lethal PCa) in patients who already have PCa. This has to be investigated in future prognostic studies.

\section{Oxidative stress-related genetic variants}

The potential effects of pro- and antioxidant nutrients/exposures on advanced PCa risk may depend on genetic variation (i.e., gene-environment interactions), specifically variation in genes encoding oxidative stress-related proteins. We prospectively investigated this in the Netherlands Cohort Study and we discovered a number of gene-environment interactions, seven of which retained significance after multiple testing adjustment. We also studied associations between the oxidative stress-related gene variants and advanced PCa risk and found that a catalase SNP was marginally associated. The positive findings in our study highlight that the oxidative stress pathway may play a role in advanced PCa development and this should motivate other researchers to continue to investigate the oxidative stress-PCa hypothesis. Future research should include gene-association studies (that focus on additional candidate SNPs) but also mechanistic studies. Mechanistic studies are important to discover how oxidative stress-related genes and exposures may have a joint effect. For example, most gene-environment interactions found in our study were difficult to interpret 
because they did not involve a dose-response pattern that involved a distinct decrease or increase in risk over exposure categories, as we hypothesized.

Findings from gene-environment interaction research can potentially be used to identify genetically susceptible individuals, for example subjects that are expected to benefit more or less from a specific (e.g., dietary) intervention. This could lead to genetic tests or tools that can help clinical decision making. However, there are several open questions that remain to be answered before findings from gene-environment interaction research can be brought to practice (i.e., costs, ethical issues, feasibility, false positives). Currently, there are a number of ongoing projects aimed at incorporating genotyping data to make dietary and other types of recommendations [14]. Whether these initiatives are successful remains to be demonstrated in the future. Our type of analysis forms the basis for these initiatives.

In conclusion, this text shows examples of how the scientific findings from this thesis can potentially be used in practice to create value. Further research is needed to carefully explore the opportunities for knowledge valorization.

\section{References}

1. Hurst R, Hooper L, Norat $T$, et al. Selenium and prostate cancer: systematic review and meta-analysis. The American journal of clinical nutrition 2012;96(1):111-22.

2. cijfersoverkanker.nl [homepage on the internet]. Nederlandse Kankerregistratie [cited 2 June 2014]. Available from: http://www.cijfersoverkanker.nl/selecties/Incidentie_prostaatkanker/ img534e82187ec19.

3. nationaalkompas.nl [homepage on the internet]. Rijksinstituut voor Volksgezondheid en Milieu [cited 19 June 2014]. Available from: http://www.nationaalkompas.nl/gezondheid-en-ziekte/ziekten-enaandoeningen/kanker/prostaatkanker/welke-zorg-gebruiken-patienten-en-kosten/.

4. Bostwick DG, Burke HB, Djakiew D, et al. Human prostate cancer risk factors. Cancer 2004;101(10 Suppl):2371-490.

5. Gronberg H. Prostate cancer epidemiology. Lancet 2003;361(9360):859-64.

6. Bostwick DG, Grignon DJ, Hammond ME, et al. Prognostic factors in prostate cancer. College of American Pathologists Consensus Statement 1999. Arch Pathol Lab Med 2000;124(7):995-1000.

7. cijfersoverkanker.nl [homepage on the internet]. Nederlandse Kankerregistratie [cited 2 June 2014]. Available from: https://www.kanker.nl/bibliotheek/prostaatkanker/wat-is/1095-cijfers-overprostaatkanker.

8. Rayman MP. Selenium and human health. Lancet 2012;379(9822):1256-68.

9. Kristal AR, Darke AK, Morris JS, et al. Baseline Selenium Status and Effects of Selenium and Vitamin E Supplementation on Prostate Cancer Risk. J Natl Cancer Inst 2014; 10.1093/jnci/djt456.

10. Makela AL, Nanto V, Makela $P$, et al. The effect of nationwide selenium enrichment of fertilizers on selenium status of healthy Finnish medical students living in south western Finland. Biol Trace Elem Res 1993;36(2):151-7.

11. Papp LV, Lu J, Holmgren A, et al. From selenium to selenoproteins: synthesis, identity, and their role in human health. Antioxidants \& redox signaling 2007;9(7):775-806.

12. Vance TM, Su J, Fontham ET, et al. Dietary antioxidants and prostate cancer: a review. Nutr Cancer 2013;65(6):793-801.

13. Wei MY, Giovannucci EL. Lycopene, Tomato Products, and Prostate Cancer Incidence: A Review and Reassessment in the PSA Screening Era. J Oncol 2012;2012:271063.

14. Mathias RA, Pani V, Chilton FH. Genetic Variants in the FADS Gene: Implications for Dietary Recommendations for Fatty Acid Intake. Curr Nutr Rep 2014;3(2):139-148. 


\section{Etcetera}




\section{Dankwoord}

Ik wil graag iedereen bedanken die mij direct of indirect heeft geholpen met mijn promotieonderzoek.

Bas en Piet. Jullie hebben dit project mogelijk gemaakt. Ik ben zeer blij dat jullie mij de kans hebben gegeven om op dit boeiende en tevens actuele onderwerp te promoveren. Wij hebben de afgelopen vier jaar nauw samengewerkt en ik heb hierbij veel van jullie geleerd. Ik wil jullie graag bedanken voor de fijne samenwerking, de vele wetenschappelijke discussies, en de steun en het vertrouwen.

Frederik-Jan. Ik wil jou, mijn tweede promotor, bedanken voor de plezierige samenwerking. Hartelijk dank ook aan jouw team voor het uitvoeren van de DNAisolatie en het voorbereiden van de genotypering.

Leo. Jij hebt een belangrijke rol gespeeld in mijn onderzoek door het genotyperingsproject te coördineren. Niemand had dit beter kunnen doen!

Anne en Nadine. Mijn allerliefste kamergenoten, jullie zijn geweldig! Waar ik in de toekomst ook zal werken, ik zal jullie missen!

Het NLCS-team. Mijn onderzoek was niet mogelijk geweest zonder jullie uitstekende voorbereidende werk en hulp. Enorm bedankt!

Epidemiologie-collega's. Ik heb heel graag met jullie samengewerkt. Bedankt voor de fijne tijd in Maastricht. De jonge garde wil ik in het bijzonder bedanken voor de vele leuke (jong-epid) uitjes: PhD-tour, Belgische bierproefavond, stappen, pub-quiz,en de vele etentjes (thuis of op restaurant). De Sinterklaas-pakjesavond was minder mijn ding, maar toch ook bedankt hiervoor ;).

Janet and the team in Seattle. My internship at the 'Hutch' has been one of the highlights of my brief scientific career. I cannot thank you enough for this great opportunity!

Mauro en Kurt. Het doet me veel plezier dat jullie mijn paranimfen willen zijn.

Mama en papa. Bedankt dat jullie mij altijd hebben gesteund!

Stefanie. Tot slot wil ik jou, mijn favoriete collega, bedanken. Jij bent natuurlijk het beste wat dit promotieonderzoek mij heeft opgeleverd. 


\section{About the author}

Milan Geybels was born on October 1, 1985 in Herk-de-Stad, Belgium. After completing secondary school (Spectrumcollege, Lummen) in 2003, he studied Biotechnology (Bachelor; PXL, Hasselt) and Biomedical Sciences (Bachelor and Master; Hasselt University, Hasselt). In 2010, he obtained his Master's degree in Biomedical Sciences. After that he started his PhD research at Maastricht University (2010-2014), the results of which are described in the present thesis. Milan performed an internship of five months at the Fred Hutchinson Cancer Research Center, Seattle, US (Public Health Sciences Division), in 2012. This internship was supported by a grant from the Dutch Cancer Society (KWF-stagebeurs voor studenten). In 2014, Milan was awarded a four-year post-doctoral fellowship from the Dutch Cancer Society (translationeel en toegepast fellowship) to continue his research. 


\section{List of publications}

\section{Appeared}

- Geybels MS, van den Brandt PA, Schouten LJ, van Schooten FJ, van Breda SG, Rayman MP, Green FR, Verhage BAJ. Selenoprotein gene variants, toenail selenium levels, and risk of advanced prostate cancer. J Natl Cancer Inst. 2014;106(3):dju003.

- Geybels MS, Verhage BAJ, van Schooten FJ, Goldbohm RA, van den Brandt PA. Advanced prostate cancer risk in relation to toenail selenium levels. J Natl Cancer Inst. 2013;105(18):1394-401.

- Geybels MS, Verhage BAJ, van Schooten FJ, Goldbohm RA, van den Brandt PA. Dietary flavonoid intake, black tea consumption, and risk of overall and advanced stage prostate cancer. Am J Epidemiol. 2013;177(12):1388-98.

- Geybels MS, Hutter CM, Kwon EM, Ostrander EA, Fu R, Feng Z, Stanford JL, Peters $U$. Variation in selenoenzyme genes and prostate cancer risk and survival. Prostate. 2013;73(7):734-42.

- Geybels MS, Wright JL, Holt SK, Kolb S, Neuhouser ML, Stanford JL. Statin use in relation to prostate cancer outcomes in a population-based patient cohort study. Prostate. 2013;73(11):1214-22.

- Geybels MS, Wright JL, Neuhouser ML, Stott-Miller M, Stanford JL. Coffee and tea consumption in relation to prostate cancer prognosis. Cancer Causes Control. 2013;24(11):1947-54.

- Geybels MS, Neuhouser ML, Stanford JL. Associations of tea and coffee consumption with prostate cancer risk. Cancer Causes Control. 2013;24(5):941-8.

- Geybels MS, Verhage BAJ, van Schooten FJ, van den Brandt PA. Measures of combined antioxidant and pro-oxidant exposures and risk of overall and advanced stage prostate cancer. Ann Epidemiol. 2012;22(11):814-20.

\section{Submitted}

- Geybels MS, van den Brandt PA, van Schooten FJ, Verhage BAJ. Oxidative stress-related genetic variants, pro- and antioxidant intake and status, and advanced prostate cancer risk. 
